\title{
The $\operatorname{AdS}_{5} \times S^{5}$ superstring in light-cone gauge and its Bethe equations
}

\author{
Sergey Frolov ${ }^{1,3}$, Jan Plefka ${ }^{2}$ and Marija Zamaklar ${ }^{1}$ \\ ${ }^{1}$ Max-Planck-Institut für Gravitationsphysik, Albert-Einstein-Institut, Am Mühlenberg 1, \\ D-14476 Potsdam, Germany \\ ${ }^{2}$ Humboldt-Universität zu Berlin, Institut für Physik, Newtonstraße 15, D-12489 Berlin, \\ Germany \\ E-mail: frolovs@aei.mpg.de,plefka@physik.hu-berlin.de and marzam@aei.mpg.de
}

Received 25 April 2006, in final form 5 July 2006

Published 27 September 2006

Online at stacks.iop.org/JPhysA/39/13037

\begin{abstract}
We use the uniform light-cone gauge to derive an exact gauge-fixed Lagrangian and light-cone Hamiltonian for the Green-Schwarz superstring in $\mathrm{AdS}_{5} \times \mathrm{S}^{5}$. We then quantize the theory perturbatively in the near plane wave limit, and compute the leading $1 / J$ correction to a generic string state from the rank-1 subsectors. These investigations enable us to propose a new set of light-cone Bethe equations for the quantum string. The equations have a simple form and yield the correct spinning string and flat space limits. Finally, we clarify the notion of closed sectors in string theory by proving the existence of perturbative effective string Hamiltonians which are direct analogues of (all-loop) dilatation operators in the dual $\mathcal{N}=4$ gauge theory.
\end{abstract}

PACS numbers: $11.15 .-\mathrm{q}, 11.25 .-\mathrm{w}, 11.30 .-\mathrm{j}$

\section{Introduction}

The quantization of the Green-Schwarz superstring [1] in the $\mathrm{AdS}_{5} \times \mathrm{S}_{5}^{5}$ background and the determination of its quantum spectrum continues to be one of the great challenges in string theory. The $\mathrm{AdS}_{5} \times \mathrm{S}^{5}$ spacetime background is distinguished by the fact that it constitutes one of the three maximally supersymmetric solutions of ten-dimensional type IIB supergravity [2], with the other two being flat Minkowski space and the IIB plane wave geometry [3], connected to $\mathrm{AdS}_{5} \times \mathrm{S}^{5}$ through suitable Penrose limits [4]. While the superstring spectrum in the latter two backgrounds is straightforwardly attainable [5] (at least in a light-cone gauge) the situation is considerably more involved for $\mathrm{AdS}_{5} \times \mathrm{S}^{5}$. Next to these structural issues, the greatest interest in the $\mathrm{AdS}_{5} \times \mathrm{S}^{5}$ quantum string stems from the AdS/CFT duality conjecture

3 Also at SUNYIT, Utica, USA and Steklov Mathematical Institute, Moscow. 
[6-8], asserting the equivalence of the string spectrum to the spectrum of scaling dimensions of composite, gauge-invariant operators in the dual $\mathcal{N}=4 U\left(N_{c}\right)$ super Yang-Mills theory in the large $N_{c}$ limit.

Here very important progress has been made during recent years building on the key concept of integrability ${ }^{4}$. On the gauge theory side, it has been established that in planar perturbation theory the dilatation operator [11], whose spectrum yields the desired scaling dimensions of composite, gauge-invariant operators, is isomorphic to the Hamiltonian of an integrable quantum spin chain [12] $]^{5}$. Here integrability secures the existence of a Bethe ansatz which enables one to reformulate the quantum spectral problem into the solution of a set of non-linear algebraic equations, the Bethe equations. These insights, at present firmly established up to the three-loop order in the 't Hooft coupling $\lambda:=g_{\mathrm{YM}} N_{c}^{2}[13,14]$ in certain closed subsectors of the full $P S U(2,2 \mid 4)$ symmetry group, led to the formulation of an exciting conjecture on the all-loop structure of these Bethe equations [15]. This conjecture has been by now extended to the full $\operatorname{PSU}(2,2 \mid 4)$ case in the gauge theory [16] and is believed to hold in an asymptotic sense, where the classical scaling dimension of the operator in question determines the loop order to which a prediction is made by the Bethe equations. Moreover, in the prominent minimal compact, bosonic subsector of $S U$ (2) the conjectured gauge theory Bethe equations [15] were recently shown to arise microscopically from the well-known Hubbard model at half filling [17]. Whether this surprising connection is indeed fully realized beyond three loops remains to be seen; for this a four-loop computation on the gauge theory side would have to be performed.

In view of these promising developments on the gauge theory side, it is clear that we need to increase our knowledge of the quantum string spectrum as well.

While it is unclear at present how to attack the question of the exact spectrum, important progress has been made by mapping out the quantum spectrum perturbatively around limiting, solvable islands within the unknown full $\mathrm{AdS}_{5} \times \mathrm{S}^{5}$ territory. One very well-studied such island is the IIB plane wave background, obtainable as a limit of $\mathrm{AdS}_{5} \times \mathrm{S}^{5}$ when a single angular momentum $J$ on the $S^{5}$ becomes large [18]. Here the first $1 / J$ corrections to the spectrum have been established in a series of papers by Callan et al in [19]. A wealth of islands is obtained by applying the semiclassical approach [20], and looking at classical spinning string solutions where several spins on $\mathrm{AdS}_{5} \times \mathrm{S}^{5}$ and angular momenta on the $\mathrm{S}^{5}$ become large [21]. In this limit, the classical string energies can be shown to provide the leading contribution to the true quantum spectrum as long as at least one angular momentum on the $S^{5}$ becomes large. Moreover, for a number of explicit solutions quantum fluctuation expansions about them have been performed [22]. A further known but not so well-studied island is the large radius limit of the $\mathrm{AdS}_{5} \times \mathrm{S}^{5}$ geometry limiting to flat Minkowski space. Here one presently only knows the leading $\lambda^{1 / 4} \sqrt{|n|}$ behaviour of the spectrum [7]; corrections in the $\lambda \rightarrow \infty$ expansion are an unknown territory.

As a matter of fact, all these computations of the quantum spectrum in the various limits which allow for an extrapolation to weak coupling $\lambda \ll 1$ display a worrisome disagreement with the gauge theory results at the three-loop order, which might be related to an order of limits problem [15]. Resolving this issue is a pressing problem for the AdS/CFT conjecture.

The sigma-model describing classical $\mathrm{AdS}_{5} \times \mathrm{S}^{5}$ strings [1] is an integrable model [23] at the classical level. One certainly hopes integrability to persist also in the quantum theory, although it is unclear at present how this could be precisely implemented. Inspired by the all-loop Bethe ansatz conjectures on the gauge theory side and being aware of the data obtained

\footnotetext{
4 For a list of reviews, see [9].

5 While integrability is know to be broken beyond the planar level, it seems to be preserved if one is focusing on the specific set of most probable string splitting channels [10].
} 
in the plane wave, flat space and spinning string limits, Arutyunov, Staudacher and one of the present authors were able to write down a set of quantum string Bethe equations [24] which are structurally very similar to the gauge theory equations of [15], differing by a so-called dressing factor which depends on (an infinite set of) undetermined functions of $\lambda$ and thus taking into account the three-loop discrepancies. These functions should be determined by comparison with quantum string data. First steps in this direction have been performed in $[22,25,26]$. The quantum string Bethe equations of [24] have also been generalized to the full $P S U(2,2 \mid 4)$ setting in $[16]^{6}$.

So on the string side two central questions arise: (1) Is the quantum string spectrum indeed described by this (or a similar) set of Bethe equations? (2) How do such Bethe equations arise at all from a treatment of the quantum string?

Addressing these questions is a complicated problem, and the present paper sets the stage for an extension of the above-mentioned perturbative studies of the quantum string spectrum by providing a novel, economic gauge-fixed description of the full $\mathrm{AdS}_{5} \times \mathrm{S}^{5}$ system. We establish the exact form of the action in the novel uniform light-cone gauge, recently introduced in a truncation to the $S U(1 \mid 1)$ subsector [28]. This gauge choice generalizes the phase-space light-cone gauge of Goddard et al [29] to curved backgrounds. It amounts to rewriting the string action in a first-order form and introducing the light-cone coordinates $x_{ \pm}:=(\phi \pm t) / 2$, where $t$ is the global time coordinate of $\mathrm{AdS}_{5}$ conjugate to the energy $E$, and $\phi$ is an angle of the $S^{5}$ whose conjugate variable is the angular momentum $J .^{7}$ The gauge consists of fixing $x_{+}=\tau$ and $p_{+}=P_{+}=$const where $p_{+}$is conjugate to $x_{-}$, along with a convenient fixing of the local fermionic $\kappa$-symmetry to be discussed.

We establish an exact first-order form of the superstring action in this gauge, consisting of a kinetic term which determines the (complicated) Poisson structure of the theory and an exact world-sheet Hamiltonian $H_{\mathrm{lc}}=-P_{-}$. In order to perform a perturbative quantization of the system about suitable limits, one needs to expand the Lagrangian in the number of physical (transverse) fields. In addition, one needs to perform a field redefinition in order to secure the standard Poisson structure and hence the canonical commutation relations. We perform this program explicitly for the case of the near plane wave limit, which in the uniform light-cone gauge amounts to taking the $P_{+} \rightarrow \infty$ limit with $\lambda / P_{+}^{2}$ held fixed, and expanding the Lagrangian up to the quartic order in transverse fields.

The resulting world-sheet Hamiltonian $H_{\mathrm{lc}}$ and the parameter $P_{+}$are related to the global energy $E$ and angular momentum $J$ via

$$
H_{\mathrm{lc}}=-P_{-}=E-J \quad P_{+}=E+J .
$$

Since $H_{\mathrm{lc}}$ itself is a function of $P_{+}$, which one determines in perturbation theory, one obtains an equation $E=J+H_{\mathrm{lc}}(E+J)$. Solving this in turn for $E$ yields the energy $E=E(J)$. This repackaging of the spectral problem for $E$ appears to be very natural from the string viewpoint. Indeed, one easily establishes that the first $1 / P_{+}$correction in the closed rank- 1 subsectors of the world-sheet energy $H_{\mathrm{lc}}$ is determined by a universal expression multiplied by $\mathfrak{s}$ with $\mathfrak{s}=\{1,0,-1\}$ for $\{\mathfrak{s u}(2), \mathfrak{s u}(1 \mid 1), \mathfrak{s l}(2)\}$ respectively.

6 An alternative approach towards the quantum string spectrum not rooted in gauge theory insights has been pursued in [27]. Here the focus is on the existence of the $S$-matrix.

7 Let us mention that in contrast to flat space where all null geodesics are equivalent, in $\operatorname{AdS}_{5} \times \mathrm{S}^{5}$, there are two inequivalent sets of null geodesics: one corresponding to a particle orbiting around the big circle on $\mathrm{S}^{5}$, while not moving in $\mathrm{AdS}_{5}$ and one where the particle moves only in the radial direction of $\mathrm{AdS}_{5}$ in global coordinates (i.e. parallel to the boundary, in the Poincare coordinates). These two geodesics in turn lead to two possible choices of the light-cone coordinates, and hence to two different light-cone gauge fixings. The latter coordinates were used in [38]. This choice is natural from the perspective of reaching the flat space limit as a Penrose limit around this geodesic yields flat space. The former coordinates were used in [5] and are natural for the purposes of reaching the plane wave limit. 
Interestingly, the energy shifts for the $H_{\mathrm{lc}}$ eigenvalues also follow from a rather simple set of light-cone Bethe equations of the form ${ }^{8}$

$$
\exp \left(\mathrm{i} p_{k} \frac{P_{+}+\mathfrak{s} M}{2}\right)=\prod_{j=1, j \neq k}^{M}\left(\frac{x_{k}^{+}-x_{j}^{-}}{x_{k}^{-}-x_{j}^{+}}\right)^{\mathfrak{s}},
$$

where $p_{k}$ denote the quasi-momenta, $M$ is the number of string oscillator excitations, and the variables $x_{k}^{ \pm}=x^{ \pm}\left(p_{k}\right)$ are those introduced in $[15,30]$ related to the quasi-momenta as

$$
x^{ \pm}(p)=\frac{1}{4}\left(\cot \frac{p}{2} \pm \mathrm{i}\right)\left(1+H_{\mathrm{lc}}(p)\right) .
$$

Note that this definition depends on the dispersion relation of the light-cone system $H_{\mathrm{lc}}(p)$. To the order we have computed $\left(\mathcal{O}\left(1 / P_{+}\right)\right)$it is given by $H_{\mathrm{lc}}(p)=\sqrt{1+\frac{\lambda}{4 \pi^{2}} p^{2}}$. This dispersion relation will be corrected at higher orders in the $1 / P_{+}$expansion. However, the simplest guess for the all-order structure inspired by the gauge theory Bethe ansätze is clearly

$$
H_{\mathrm{lc}}(p)=\sqrt{1+\frac{\lambda}{\pi^{2}} \sin ^{2}\left(\frac{p}{2}\right)} \text {. }
$$

A necessary condition on our light-cone Bethe equations (1.2) is the correct behaviour both in the spinning string and strong coupling limits. This is explicitly demonstrated in section 8 . The novel feature of this set of Bethe equations is that the exponent on the left-hand side is not an integer any longer, as $P_{+}=E+J$ where $E$ is the global energy we wish to determine in the end. Moreover, the dressing factor which was present for the quantum string equations [24] is now absent. It may appear, however, at higher orders in $1 / P_{+}$.

Much of the material presented in this paper is of a rather technical nature, which we have tried to delegate to the appendices as much as possible. In the main text, we present the logic and the flow in the construction and uniform light-cone gauge fixing of the superstring on $\mathrm{AdS}_{5} \times \mathrm{S}^{5}$. The leading corrections in the near plane wave expansion are presented in great detail, along with a discussion of the most prominent closed subsectors and their energy shifts. Here we reproduce the results of [19] in a very economic fashion. We also comment on the emergence of effective Hamiltonians for the closed subsectors, which are the direct analogues of the dilatation operators in the parallel subsectors in the gauge theory. In the final section, we present the derivation of the light-cone Bethe equations stated above and show that its thermodynamic and strong coupling limits are in agreement with previous results.

\section{Review of the superstring on $\mathrm{AdS}_{5} \times \mathrm{S}^{5}$}

In this section, we review the structure of the Green-Schwarz superstring action in the $\mathrm{AdS}_{5} \times \mathrm{S}^{5}$ spacetime geometry following closely the discussion in [31,32]. The superstring is formulated as a two-dimensional non-linear sigma-model whose target space is given by the coset manifold [1]

$$
\frac{P S U(2,2 \mid 4)}{S O(4,1) \times S O(5)} \text {. }
$$

The full action is given by the sum of the non-linear sigma-model action and a topological Wess-Zumino term which is fixed uniquely by requiring $\operatorname{PSU}(2,2 \mid 4)$ and $\kappa$-symmetry invariance.

8 Note that the wording 'light cone' used here does not refer to the gauge used to derive the world-sheet Hamiltonian. In the spinning string limit, equations (1.2) reduce to the integral equations of [44, 45] which are derived from the gauge unfixed sigma-model action. The main reason why our equations have a different form than those of [24] is because they are diagonalizing a different (but equivalent) infinite set of charges. For example, the role of the global energy $E$ is replaced by the light-cone energy $-P_{-}$. 
Let us first discuss some basic facts about the supergroup PSU $(2,2 \mid 4)$ that is the isometry group of the $\mathrm{AdS}_{5} \times \mathrm{S}^{5}$ superspace and the corresponding Lie superalgebra $\mathfrak{p s u}(2,2 \mid 4)$.

\subsection{Superalgebra $\mathfrak{p s u}(2,2 \mid 4)$}

A convenient description of the superalgebra $\mathfrak{s u}(2,2 \mid 4)$ is provided by $8 \times 8$ supermatrices $M$ which can be written in terms of $4 \times 4$ blocks as

$$
M=\left(\begin{array}{ll}
A & X \\
Y & D
\end{array}\right) .
$$

Here the matrices $A$ and $D$ are Grassmann even and $X, Y$ are Grassmann odd. The superalgebra $\mathfrak{s u}(2,2 \mid 4)$ is singled out by requiring $M$ to have vanishing supertrace $\operatorname{str} M=\operatorname{tr} A-\operatorname{tr} D=0$ and to satisfy the following reality condition:

$$
H M+M^{\dagger} H=0 \text {. }
$$

The choice of the Hermitian matrix $H$ is not unique and we choose $H$ to be of the diagonal form

$$
H=\left(\begin{array}{ll}
\Sigma & 0 \\
0 & \mathbb{I}
\end{array}\right)
$$

where $\Sigma$ is the following matrix,

$$
\Sigma=\left(\begin{array}{cccc}
1 & 0 & 0 & 0 \\
0 & 1 & 0 & 0 \\
0 & 0 & -1 & 0 \\
0 & 0 & 0 & -1
\end{array}\right)
$$

and II denotes the identity matrix of the corresponding dimension. This choice of $H$ makes it obvious that the bosonic matrices $A$ and $D$ belong to the algebras $\mathfrak{u}(2,2)$ and $\mathfrak{u}(4)$ respectively. The condition (2.3) also implies that the fermionic matrices $X$ and $Y$ are conjugated to each other via the relation

$$
Y=-X^{\dagger} \Sigma \text {. }
$$

Only the supertraceless combination of the two $\mathfrak{u}(1)$ generators of $\mathfrak{u}(2,2)$ and $\mathfrak{u}(4)$ belongs to $\mathfrak{s u}(2,2 \mid 4)$. It is represented by the anti-Hermitian matrix iII. Thus, the bosonic subalgebra of $\mathfrak{s u}(2,2 \mid 4)$ is

$$
\mathfrak{s u}(2,2) \oplus \mathfrak{s u}(4) \oplus \mathfrak{u}(1) .
$$

The superalgebra $\mathfrak{p} \mathfrak{s u}(2,2 \mid 4)$ is defined as the quotient algebra of $\mathfrak{s u}(2,2 \mid 4)$ over this $\mathfrak{u}(1)$ factor. It has no realization in terms of $8 \times 8$ supermatrices.

The construction of the superstring action uses the $\mathbb{Z}_{4}$ grading of the superalgebra $\mathfrak{s u}(2,2 \mid 4)$ defined by the automorphism $M \rightarrow \Omega(M)$ with

$$
\Omega(M)=\left(\begin{array}{cc}
K A^{t} K & -K Y^{t} K \\
K X^{t} K & K D^{t} K
\end{array}\right),
$$

where $A^{t}$ is the usual transpose of $A$ and we choose the $4 \times 4$ matrix $K$ satisfying $K^{2}=-I$ to be

$$
K=\left(\begin{array}{cccc}
0 & 1 & 0 & 0 \\
-1 & 0 & 0 & 0 \\
0 & 0 & 0 & 1 \\
0 & 0 & -1 & 0
\end{array}\right)
$$


Any matrix $M$ from $\mathfrak{s u}(2,2 \mid 4)$ can then be decomposed into the sum

$$
M=\underbrace{M^{(0)}+M^{(2)}}_{\text {even }}+\underbrace{M^{(1)}+M^{(3)}}_{\text {odd }},
$$

where every matrix $M^{(p)}$ is an eigenstate of $\Omega$

$$
\Omega\left(M^{(p)}\right)=i^{p} M^{(p)} .
$$

Explicitly, the matrices $M^{(p)}$ are given by

$$
\begin{aligned}
& M^{(0)}=\frac{1}{4}\left(M+\Omega(M)+\Omega^{2}(M)+\Omega^{3}(M)\right)=\frac{1}{2}\left(\begin{array}{cc}
A+K A^{t} K & 0 \\
0 & D+K D^{t} K
\end{array}\right), \\
& M^{(2)}=\frac{1}{4}\left(M-\Omega(M)+\Omega^{2}(M)-\Omega^{3}(M)\right)=\frac{1}{2}\left(\begin{array}{cc}
A-K A^{t} K & 0 \\
0 & D-K D^{t} K
\end{array}\right), \\
& M^{(1)}=\frac{1}{4}\left(M-\mathrm{i} \Omega(M)-\Omega^{2}(M)+\mathrm{i} \Omega^{3}(M)\right)=\frac{1}{2}\left(\begin{array}{cc}
0 & X+i K Y^{t} K \\
Y-i K X^{t} K & 0
\end{array}\right), \\
& M^{(3)}=\frac{1}{4}\left(M+\mathrm{i} \Omega(M)-\Omega^{2}(M)-\mathrm{i} \Omega^{3}(M)\right)=\frac{1}{2}\left(\begin{array}{cc}
0 & X-i K Y^{t} K \\
Y+i K X^{t} K & 0
\end{array}\right) .
\end{aligned}
$$

It is not difficult to check by using these formulae that the matrices $M^{(0)}$ form the $\mathfrak{s o}(4,1) \times \mathfrak{s o}(5)$ subalgebra which we wish to $\bmod$ out in the coset. We also see that the matrices $M^{(1,3)}$ contain the odd matrices. Splitting $M$ into Grassmann even and odd parts

$$
M=M_{\text {even }}+M_{\text {odd }}, \quad M_{\text {even }}=\left(\begin{array}{cc}
A & 0 \\
0 & D
\end{array}\right), \quad M_{\text {odd }}=\left(\begin{array}{cc}
0 & X \\
Y & 0
\end{array}\right),
$$

one can also rewrite the explicit expressions for $M^{(p)}$ in the following form,

$M^{(0)}=\frac{1}{2}\left(M_{\text {even }}+K_{8} M_{\text {even }}^{t} K_{8}\right), \quad M^{(2)}=\frac{1}{2}\left(M_{\text {even }}-K_{8} M_{\text {even }}^{t} K_{8}\right)$,

$M^{(1)}=\frac{1}{2}\left(M_{\text {odd }}+\mathrm{i} \widetilde{K}_{8} M_{\text {odd }}^{t} K_{8}\right), \quad M^{(3)}=\frac{1}{2}\left(M_{\text {odd }}-i \widetilde{K}_{8} M_{\text {odd }}^{t} K_{8}\right)$,

where $K$ and $\widetilde{K}$ are defined as

$$
K_{8}=\left(\begin{array}{cc}
K & 0 \\
0 & K
\end{array}\right), \quad \widetilde{K}_{8}=\left(\begin{array}{cc}
K & 0 \\
0 & -K
\end{array}\right) .
$$

The orthogonal complement $M^{(2)}$ of $s o(4,1) \times \operatorname{so}(5)$ in $\mathfrak{s u}(2,2) \oplus \mathfrak{s u}(4)$ can be conveniently described as follows. In appendix A we introduce the Dirac matrices for $S O(5) \gamma_{s}, s=1,2,3,4$ and $\gamma_{5} \equiv \Sigma$, which we all take to be Hermitian. These matrices obey the relations

$$
K \gamma_{s}^{t} K=-\gamma_{s}, \quad K \Sigma^{t} K=-\Sigma,
$$

and, therefore, span the orthogonal complement to the Lie algebra $\mathfrak{s o}(5)$. The same matrices can be used to build the set of Dirac matrices for $\mathfrak{s o}(4,1)$; one takes $\left\{\mathrm{i} \Sigma, \gamma_{a}\right\}$ with $a=1,2,3,4$. Hence, we can represent any matrix $M^{(2)}$ from $\mathfrak{s u}(2,2 \mid 4)$ in the form

$$
M^{(2)}=\left(\begin{array}{cc}
\mathrm{i} t \Sigma+z_{a} \gamma_{a} & 0 \\
0 & \mathrm{i} \phi \Sigma+\mathrm{i} y_{s} \gamma_{s}
\end{array}\right)+\mathrm{i} m_{0} \mathbb{I} \equiv x_{M} \Sigma_{M}+\left(\begin{array}{cc}
\mathrm{i} t \Sigma & 0 \\
0 & \mathrm{i} \phi \Sigma
\end{array}\right)+\mathrm{i} m_{0} \mathbb{I},
$$

where $x_{M}=\left\{z_{a}, y_{s}\right\}, t, \phi$ and $m_{0}$ are real parameters of $M^{(2)}$, and the $8 \times 8$ matrices

$$
\Sigma_{M}=\left\{\left(\begin{array}{cc}
\gamma_{a} & 0 \\
0 & 0
\end{array}\right),\left(\begin{array}{cc}
0 & 0 \\
0 & i \gamma_{s}
\end{array}\right)\right\}, \quad \Sigma_{+}=\left(\begin{array}{cc}
\Sigma & 0 \\
0 & \Sigma
\end{array}\right), \quad \Sigma_{-}=\left(\begin{array}{cc}
-\Sigma & 0 \\
0 & \Sigma
\end{array}\right) .
$$

together with the $U(1)$ generator ill form a basis of $M^{(2)}$ which shall be of use in the following. 


\subsection{Lagrangian and coset element}

Consider now a group element $g$ belonging to $\operatorname{PSU}(2,2 \mid 4)$ and construct the following current,

$$
A=-g^{-1} \mathrm{~d} g=\underbrace{A^{(0)}+A^{(2)}}_{\text {even }}+\underbrace{A^{(1)}+A^{(3)}}_{\text {odd }},
$$

where we also exhibited its $\mathbb{Z}_{4}$ decomposition. By construction this current has zero curvature. The Lagrangian density for the superstring in $\operatorname{AdS}_{5} \times \mathrm{S}^{5}$ can then be written in the form $[1,33]$

$$
\mathscr{L}=-\frac{1}{2} \sqrt{\lambda} \operatorname{Str}\left(\gamma^{\alpha \beta} A_{\alpha}^{(2)} A_{\beta}^{(2)}+\kappa \epsilon^{\alpha \beta} A_{\alpha}^{(1)} A_{\beta}^{(3)}\right),
$$

which is the sum of the kinetic and the Wess-Zumino terms and $\kappa$-symmetry requires $\kappa= \pm 1$. Here we use the convention $\epsilon^{01} \equiv \epsilon^{\tau \sigma}=1$ and $\gamma^{\alpha \beta}=h^{\alpha \beta} \sqrt{-h}$ is the Weyl-invariant combination of the metric on the string world-sheet with $\operatorname{det} \gamma=-1$.

There are many different ways to parametrize the coset elements (2.1) related to each other by non-linear field redefinitions. For example, the authors of [32] considered the parametrization for the coset element

$$
g=g(\theta) g(x)
$$

where $\theta$ parametrizes the fermionic and $x$ the bosonic degrees of freedom. This form is especially convenient to analyse the global symmetries of the Lagrangian (2.20) as the symmetries act linearly on the fermionic variables $\theta$. Due to this the fermions $\theta$ are charged under any $U(1)$ subgroup of $P S U(2,2 \mid 4)$ and in particular under the subgroups generated by shifts of the global time coordinate $t$ of $\mathrm{AdS}_{5}$ and of an angle variable $\phi$ of $\mathrm{S}^{5}$. On the other hand, as was discussed in [28], to impose the light-cone gauge it is convenient to use fields neutral under these two $U(1)$ subgroups. This requires us to redefine the fermions by performing a similarity transformation of the matrix $g(\theta)[34]^{9}$. The result of this transformation is equivalent to choosing a different coset element from the start, namely we choose the element to be of the form

$$
g(\chi, x, t, \phi)=\Lambda(t, \phi) g(\chi) g(x) .
$$

Here $x^{M}=\left\{z_{a}, y_{s}\right\}, a, s=1, \ldots, 4$, and the coordinates $t, z_{a}$ and $\phi, y_{s}$ parametrize $\mathrm{AdS}_{5}$ and $S^{5}$, respectively. The even matrices $\Lambda(t, \phi)$ and $g(x)$ describe an embedding of $\operatorname{AdS}_{5} \times S^{5}$ into $S U(2,2) \times S U(4)$ and $g(\chi)$ is a matrix which incorporates the 32 fermionic degrees of freedom. The matrix $g(x)$ and the diagonal matrix $\Lambda$ are defined as

$$
\begin{aligned}
& g(x)=\left(\begin{array}{cc}
g_{a}(z) & 0 \\
0 & g_{s}(y)
\end{array}\right), \\
& g_{a}(z)=\frac{1}{\sqrt{1-\frac{z^{2}}{4}}}\left(1+\frac{1}{2} z_{a} \gamma_{a}\right), \quad g_{s}(y)=\frac{1}{\sqrt{1+\frac{y^{2}}{4}}}\left(1+\frac{\mathrm{i}}{2} y_{s} \gamma_{s}\right),
\end{aligned}
$$

and

$\Lambda(t, \phi)=\exp \left[\frac{\mathrm{i}}{2} t\left(\begin{array}{ll}\Sigma & 0 \\ 0 & 0\end{array}\right)+\frac{\mathrm{i}}{2} \phi\left(\begin{array}{ll}0 & 0 \\ 0 & \Sigma\end{array}\right)\right]=\exp \left[\frac{\mathrm{i}}{2} x_{+} \Sigma_{+}+\frac{\mathrm{i}}{2} x_{-} \Sigma_{-}\right]$,

9 In [34] a similarity transformation was used to make fermions neutral under all six $U(1)$ subgroups of $P S U(2,2 \mid 4)$ that was necessary to apply TsT-transformations to derive the Green-Schwarz action on $\gamma$-deformed $\mathrm{AdS}_{5} \times \mathrm{S}^{5}$ backgrounds $[35,36]$. 
where we have introduced the light-cone coordinates $x_{ \pm}, t=x_{+}-x_{-}$and $\phi=x_{+}+x_{-}$, and used the $8 \times 8$ matrices $\Sigma_{ \pm}$of (2.18).

It is not difficult to check that the following important relations are valid,

$$
\Sigma_{ \pm} g^{-1}(x)=g(x) \Sigma_{ \pm},
$$

because $\Sigma$ anticommutes with $\gamma_{a}$ and $g^{-1}(x)=g(-x)$.

Using the parametrization (2.24) the metric on the coset becomes

$$
\begin{aligned}
& \mathrm{d} s^{2}=-G_{t t}(z) \mathrm{d} t^{2}+G_{\phi \phi} \mathrm{d} \phi^{2}+\frac{1}{\left(1-\frac{z^{2}}{4}\right)^{2}} \mathrm{~d} z_{i} \mathrm{~d} z_{j}+\frac{1}{\left(1+\frac{y^{2}}{4}\right)^{2}} \mathrm{~d} y_{i} \mathrm{~d} y_{j} . \\
& G_{t t}=\left(\frac{1+\frac{z^{2}}{4}}{1-\frac{z^{2}}{4}}\right)^{2} \quad G_{\phi \phi}=\left(\frac{1-\frac{y^{2}}{4}}{1+\frac{y^{2}}{4}}\right)^{2},
\end{aligned}
$$

which shows explicitly that $t$ is the global time coordinate of $\mathrm{AdS}_{5}, \phi$ is an angle of $\mathrm{S}^{5}$, and $z_{i}$ and $y_{i}$ are the remaining coordinates of $\mathrm{AdS}_{5}$ and $\mathrm{S}^{5}$, respectively.

Finally, we choose the matrix $g(\chi)$ to be of the form

$$
g(\chi)=\chi+\sqrt{1+\chi^{2}}
$$

where the odd matrix $\chi$ is

$$
\chi=\left(\begin{array}{cc}
0 & \Theta \\
\Theta_{*} & 0
\end{array}\right), \quad \Theta_{*}=-\Theta^{\dagger} \Sigma, \quad \Theta=\left(\begin{array}{llll}
\theta_{11} & \theta_{12} & \theta_{13} & \theta_{14} \\
\theta_{21} & \theta_{22} & \theta_{23} & \theta_{24} \\
\theta_{31} & \theta_{32} & \theta_{33} & \theta_{34} \\
\theta_{41} & \theta_{42} & \theta_{43} & \theta_{44}
\end{array}\right) .
$$

Here $\theta_{i j}$ are complex fermions, and $\Theta^{\dagger}$ is the Hermitian conjugate of $\Theta$. By construction the element $g$, and $g(\chi)$ in particular, belong to the supergroup $S U(2,2 \mid 4)$. Let us stress that the fermions and the bosonic coordinates $z_{i}$ and $y_{i}$ do not transform under the $U(1)$ transformations generated by shifts of $t$ and $\phi$. The fields are charged under the four remaining $U(1)$ subgroups of $P S U(2,2 \mid 4)$. The charges are given in appendix A.

\section{Light-cone gauge}

In this section, we introduce the first-order formalism for the Green-Schwarz superstring in $\mathrm{AdS}_{5} \times \mathrm{S}^{5}$, and then, following [28], impose the uniform light-cone gauge and fix the $\kappa$-symmetry. The uniform light-cone gauge generalizes the standard phase-space light-cone gauge of [29] to a curved background, and differs from that used in [38] by the choice of the light-cone coordinates and $\kappa$-symmetry fixing. It belongs to the class of uniform gauges used to study the dynamics of spinning strings in $\mathrm{AdS}_{5} \times \mathrm{S}^{5}[39,41]$.

\subsection{First-order formalism}

The simplest way to impose a light-cone gauge is to introduce momenta canonically conjugate to the coordinates $t$ and $\phi$ (or, equivalently, to the light-cone coordinates $x_{ \pm}$). ${ }^{10}$ In the case of superstrings in $\mathrm{AdS}_{5} \times \mathrm{S}^{5}$ it is difficult to find the momenta because of a nontrivial interaction between bosonic and fermionic fields. A better way to proceed is to introduce a Lie-algebra valued auxiliary field $\pi$, and rewrite the superstring Lagrangian (2.20) in the form

$\mathscr{L}=-\operatorname{Str}\left(\pi A_{0}^{(2)}+\kappa \frac{\sqrt{\lambda}}{2} \epsilon^{\alpha \beta} A_{\alpha}^{(1)} A_{\beta}^{(3)}-\frac{1}{2 \sqrt{\lambda} \gamma^{00}}\left(\pi^{2}+\lambda\left(A_{1}^{(2)}\right)^{2}\right)+\frac{\gamma^{01}}{\gamma^{00}}\left(\pi A_{1}^{(2)}\right)\right)$.

\footnotetext{
10 This is the best way to impose any uniform gauge where a momentum is distributed uniformly along a string.
} 
It is easy to see that if we solve the equations of motion for $\pi$ and substitute the solution back into (3.1) one obtains (2.20). The last two terms in (3.1) yield the Virasoro constraints

$$
\begin{aligned}
& C_{1}=\operatorname{Str}\left(\pi^{2}+\lambda\left(A_{1}^{(2)}\right)^{2}\right)=0, \\
& C_{2}=\operatorname{Str} \pi A_{1}^{(2)}=0,
\end{aligned}
$$

which are to be solved after imposing the light-cone gauge and fixing the $\kappa$-symmetry.

Without loss of generality we can assume that $\pi$ belongs to the subspace $M^{(2)}$ of $\mathfrak{s u}(2,2 \mid 4)$, as the other components in the $\mathbb{Z}_{4}$ grading decouple. It therefore admits the following decomposition (compare (2.18)),

$$
\pi=\pi^{(2)}=\frac{\mathrm{i}}{4} \pi_{+} \Sigma_{+}+\frac{\mathrm{i}}{4} \pi_{-} \Sigma_{-}+\frac{1}{2} \pi_{M} \Sigma_{M}+\pi_{0} \mathrm{iII},
$$

where $\Sigma^{M}$ are given by equation (2.18). It is obvious that since $A_{\alpha}^{(2)}$ belongs to the superalgebra $\mathfrak{s u}(2,2 \mid 4)$, Str $A_{\alpha}^{(2)}=0$, the variable $\pi_{0}$ does not contribute to the Lagrangian. The decomposition (3.4) secures the following identity:

$$
\operatorname{Str} \pi A_{\alpha}^{(2)}=\operatorname{Str} \pi^{(2)} A_{\alpha}=\operatorname{Str} \pi A_{\alpha} .
$$

The fields $\pi_{ \pm}$are not the momenta $p_{ \pm}$canonically conjugate to $x_{\mp}$ but they may be expressed in terms of $p_{ \pm}$. Before doing so let us impose the $\kappa$-symmetry gauge conditions, which simplify all expressions dramatically.

\subsection{Fixing $\kappa$-symmetry}

A key property of the Green-Schwarz action is its invariance under the fermionic $\kappa$-symmetry that halves the number of fermionic degrees of freedom. A $\kappa$-symmetry gauge should be compatible with the bosonic gauge imposed, and analysing the $\kappa$-symmetry transformations (which can be extracted from [1,33]) for the Green-Schwarz superstring action (3.1) one can show that in the case of the uniform light-cone gauge $\kappa$-symmetry can be fixed by choosing the fermion $\Theta$ of (2.29) to be of the form

$$
\Theta=\left(\begin{array}{cccc}
0 & 0 & \theta_{13} & \theta_{14} \\
0 & 0 & \theta_{23} & \theta_{24} \\
\theta_{31} & \theta_{32} & 0 & 0 \\
\theta_{41} & \theta_{42} & 0 & 0
\end{array}\right) .
$$

It is not difficult to check that $\Theta$ of such a form anticommutes with $\Sigma$ and therefore the gauge-fixed $\chi$ satisfies the following important relations:

$$
\Sigma_{+} \chi=-\chi \Sigma_{+}, \quad \Sigma_{-} \chi=\chi \Sigma_{-} .
$$

In fact these relations may be considered as the defining relations for the $\kappa$-symmetry gauge we have chosen and can be used instead of specifying the explicit form of $\chi$.

Taking into account that $g^{-1}(\chi)=g(-\chi)$ and these identities, one can easily show that

$$
\begin{aligned}
& g^{-1}(\chi) \Sigma_{+}=\Sigma_{+} g(\chi) \quad \Rightarrow \quad g^{-1}(\chi) \Sigma_{+} g(\chi)=\Sigma_{+} g(\chi)^{2} \\
& g^{-1}(\chi) \Sigma_{-}=\Sigma_{-} g^{-1}(\chi) \quad \Rightarrow \quad g^{-1}(\chi) \Sigma_{-} g(\chi)=\Sigma_{-}
\end{aligned}
$$

Now it is straightforward to use the coset parametrization (2.22) to compute the current (2.19) 


$$
\begin{aligned}
& \begin{array}{l}
A=A_{\text {even }}+A_{\text {odd }} \\
A_{\text {even }}=-g^{-1}(x)
\end{array}\left[\frac{\mathrm{i}}{2} \mathrm{~d} x_{+} \Sigma_{+}\left(1+2 \chi^{2}\right)+\frac{\mathrm{i}}{2} \mathrm{~d} x_{-} \Sigma_{-}\right] g(x) \\
& \quad-g^{-1}(x)\left[\sqrt{1+\chi^{2}} \mathrm{~d} \sqrt{1+\chi^{2}}-\chi \mathrm{d} \chi+\mathrm{d} g(x) g^{-1}(x)\right] g(x) . \\
& A_{\text {odd }}=-g^{-1}(x)\left[\mathrm{i} \mathrm{d} x_{+} \Sigma_{+} \chi \sqrt{1+\chi^{2}}+\sqrt{1+\chi^{2}} \mathrm{~d} \chi-\chi \mathrm{d} \sqrt{1+\chi^{2}}\right] g(x) .
\end{aligned}
$$

These formulae demonstrate explicitly the important advantage of the $\kappa$-symmetry gauge we have imposed. The odd part of the current $A$ does not depend on the light-cone coordinate $x_{-}$! It also explains the drastic simplifications that will occur in the uniform light-cone gauge in comparison to the uniform gauge $t=\tau, p_{\phi}=J$ used in previous works [19, 41]. In the gauge $x_{+}=\tau$ the odd part of $A$ depends only on the derivatives of the fermion $\chi$.

\subsection{Fixing the light-cone gauge}

After having fixed the $\kappa$-symmetry we can now proceed to express $p_{ \pm}$in terms of $\pi_{ \pm}$. To this end, omitting the Virasoro constraints, we can rewrite the Lagrangian (3.1) as follows,

$$
\mathscr{L}=p_{+} \dot{x}_{-}+\mathbf{p}_{-} \dot{x}_{+}-\operatorname{Str}\left(\pi A_{\text {even }}^{\perp}+\kappa \frac{\sqrt{\lambda}}{2} \epsilon^{\alpha \beta} A_{\alpha}^{(1)} A_{\beta}^{(3)}\right)
$$

where

$$
A_{\text {even }}^{\perp}=-g^{-1}(x)\left[\sqrt{1+\chi^{2}} \partial_{\tau} \sqrt{1+\chi^{2}}-\chi \partial_{\tau} \chi+\partial_{\tau} g(x) g^{-1}(x)\right] g(x),
$$

and the momentum $p_{+}$canonically conjugate to $x_{-}$can be easily shown to be equal to

$p_{+}=\frac{\mathrm{i}}{2} \operatorname{Str}\left(\pi \Sigma_{-} g(x)^{2}\right)=G_{+} \pi_{+}-G_{-} \pi_{-}, \quad G_{ \pm}=\frac{1}{2}\left(G_{t t}^{\frac{1}{2}} \pm G_{\phi \phi}^{\frac{1}{2}}\right)$.

The variable $\mathbf{p}_{-}$is not equal to the momentum $p_{-}$canonically conjugate to $x_{+}$. It differs from $p_{-}$by a contribution coming from the Wess-Zumino term, and is defined as follows:

$$
\mathbf{p}_{-}=\frac{\mathrm{i}}{2} \operatorname{Str}\left(\pi \Sigma_{+} g(x)\left(1+2 \chi^{2}\right) g(x)\right) \text {. }
$$

The uniform light-cone gauge is now imposed by setting [28]

$$
\begin{array}{ll}
x_{+}=\tau+\frac{m}{2} \sigma, & p_{+}=P_{+}=E+J=\mathrm{const}, \\
x_{ \pm}=\frac{1}{2}(\phi \pm t), & p_{+}=p_{\phi}-p_{t} \quad p_{-}=p_{t}+p_{\phi},
\end{array}
$$

where the spacetime energy $E$ and the angular momenta $J$ are integrals over $\sigma$ of the momenta $p_{t}$ and $p_{\phi}$ conjugate to the global AdS time $t$ and the angle $\phi$

$$
E=-\int \frac{\mathrm{d} \sigma}{2 \pi} p_{t}, \quad J=\int \frac{\mathrm{d} \sigma}{2 \pi} p_{\phi}
$$

The string winding number $m$ appears because $\phi$ is an angle variable. In what follows, we will be interested in the near plane wave limit, and, therefore, we set $m=0$. Let us stress again that the density $\mathcal{H}$ of the target space Hamiltonian is not equal to $-\mathbf{p}_{-}$. The Wess-Zumino term in (3.12) also contributes to $p_{-}$, and, therefore, to $\mathcal{H}$. 


\section{4. $P S U(2,2 / 4)$ charges}

The invariance of the Green-Schwarz action under the PSU $(2,214)$ group leads to the existence of conserved currents and charges. As was shown in [31] the conserved currents can be written in terms of $A_{\alpha}$ as follows:

$$
J^{\alpha}=\sqrt{\lambda} g(x, \theta)\left(\gamma^{\alpha \beta} A_{\beta}^{(2)}-\frac{\kappa}{2} \epsilon^{\alpha \beta}\left(A_{\beta}^{(1)}-A_{\beta}^{(3)}\right)\right) g(x, \theta)^{-1} .
$$

The conserved charges are then given by integrals over $\sigma$ of $J^{\tau}$

$$
Q=\int_{0}^{2 \pi} \frac{\mathrm{d} \sigma}{2 \pi} J^{\tau}
$$

For our purposes, it is convenient to express the charges in terms of the momenta $\pi$. To this end, we note that they satisfy the following equations of motion,

$$
\pi=\sqrt{\lambda} \gamma^{\tau \beta} A_{\beta}^{(2)}=\sqrt{\lambda} \gamma^{\tau \tau}\left(A_{\tau}^{(2)}+\frac{\gamma^{\tau \sigma}}{\gamma^{\tau \tau}} A_{\sigma}^{(2)}\right)
$$

and therefore we can express $A_{\tau}^{(2)}$ in terms of $\pi$, and substitute it into expression (3.19) for $Q$. After a simple algebra we get

$$
Q=\int_{0}^{2 \pi} \frac{\mathrm{d} \sigma}{2 \pi} g(x, \theta)\left(\pi-\sqrt{\lambda} \frac{\kappa}{2}\left(A_{\sigma}^{(1)}-A_{\sigma}^{(3)}\right)\right) g(x, \theta)^{-1}
$$

The formula can be written in a more explicit form if we take into account that

$$
A_{\sigma}^{(1)}-A_{\sigma}^{(3)}=-\mathrm{i} g(x) \widetilde{K}_{8} F_{\sigma}^{t} K_{8} g(x)^{-1},
$$

where

$$
F_{\sigma}=\sqrt{1+\chi^{2}} \partial_{\sigma} \chi-\chi \partial_{\sigma} \sqrt{1+\chi^{2}}
$$

is an odd component of the current $g^{-1}(\chi) \partial_{\sigma} g(\chi)$. Then, the $\mathfrak{p s u}(2,2 \mid 4)$ charges are

$Q=\int_{0}^{2 \pi} \frac{\mathrm{d} \sigma}{2 \pi} \Lambda g(\chi) g(x)\left(\pi+\mathrm{i} \sqrt{\lambda} \frac{\kappa}{2} g(x) \widetilde{K}_{8} F_{\sigma}^{t} K_{8} g(x)^{-1}\right) g(x)^{-1} g(\chi)^{-1} \Lambda^{-1}$.

The expression is very simple, and it has the important property that $Q$ does not have an explicit dependence on the world-sheet metric.

The combinations of components of the matrix $Q$ give charges corresponding to rotations, dilatations, supersymmetry and so on. To single out the charges one should multiply $Q$ by a corresponding $8 \times 8$ matrix, and take the supertrace

$$
Q_{\mathcal{M}}=\operatorname{Str}(Q \mathcal{M}) \text {. }
$$

The diagonal and skew-diagonal $4 \times 4$ blocks of $\mathcal{M}$ single out bosonic and fermionic charges of $\mathfrak{p s u}(2,2 \mid 4)$, respectively.

We divide all charges into two groups: kinematic and dynamic charges. Kinematic charges are those that do not depend on $x_{-}$and receive no corrections. The matrices $\mathcal{M}$ corresponding to kinematic charges are of the form

$$
\mathcal{M}_{\text {kin }}=\left(\begin{array}{cccc}
a & 0 & 0 & g \\
0 & b & f & 0 \\
0 & \tilde{f} & \tilde{a} & 0 \\
\tilde{g} & 0 & 0 & \tilde{b}
\end{array}\right)
$$

where $a, b, \tilde{a}$ and $\tilde{b}$ are $s u(2)$ matrices. This is because $\Sigma_{-}$commutes with any matrix of such a form, and therefore $x_{-}$drops out of $Q_{\mathcal{M}}$. We also add to these charges the 1.c. momentum $p_{+}$that is expressed in terms of $Q$ as follows:

$$
p_{+}=\frac{\mathrm{i}}{2} \operatorname{Str}\left(Q \Sigma_{-}\right) \text {. }
$$


One can easily check that $p_{+}$coincides with (3.14).

The matrices $\mathcal{M}$ corresponding to dynamic charges are obviously of the form

$$
\mathcal{M}_{\text {dyn }}=\left(\begin{array}{cccc}
0 & c & h & 0 \\
d & 0 & 0 & k \\
\tilde{h} & 0 & 0 & \tilde{c} \\
0 & \tilde{k} & \tilde{d} & 0
\end{array}\right),
$$

and we also should add the 1.c. momentum $p_{-}=-H_{l . c}$ that is expressed in terms of $Q$ as follows:

$$
p_{-}=\frac{\mathrm{i}}{2} \operatorname{Str}\left(Q \Sigma_{+}\right)
$$

It is not difficult to verify that $-p_{-}$coincides with the Hamiltonian (4.4).

A convenient basis of the matrices $\mathcal{M}$ is provided by the $S O(4)$ gamma matrices. It is not difficult to check that $\mathcal{M}_{\text {kin }}$ are spanned by the following set of 29 matrices,

$$
\mathcal{M}_{\text {kin }}=\left\{\Sigma_{-}, P_{2}^{ \pm} \otimes \frac{1}{2}\left[\Gamma_{a}, \Gamma_{b}\right], \sigma^{ \pm} \otimes \Gamma_{a}, \sigma^{ \pm} \otimes \Sigma \Gamma_{a}\right\},
$$

and $\mathcal{M}_{\text {dyn }}$ are spanned by the following 33 matrices,

$\mathcal{M}_{\text {dyn }}=\left\{\Sigma_{+}, P_{2}^{ \pm} \otimes \Gamma_{a}, P_{2}^{ \pm} \otimes \Sigma \Gamma_{a}, \sigma^{ \pm} \otimes \frac{1}{2}\left[\Gamma_{a}, \Gamma_{b}\right], \sigma^{ \pm} \otimes \Sigma, \sigma^{ \pm} \otimes I_{4}\right\}$.

The detailed structure of the conserved charges and their algebra will be discussed elsewhere.

\section{Gauge-fixed Lagrangian}

Now we are ready to find the light-cone gauge-fixed Lagrangian. This is a multistep procedure. First we solve equation (3.14) for $\pi_{+}\left(P_{+}, \pi_{-}\right)$. Second we solve the Virasoro constraint $C_{2}$ of equation (3.3) to find $x_{-}^{\prime}$. Finally we determine $\pi_{-}$from the second Virasoro constraint $C_{1}$ of equation (3.2). Substituting all the solutions into the Lagrangian of equation (3.12), we end up with the total gauge-fixed Lagrangian. This explicit derivation and definitions of all the quantities used to write it down may be found in appendix B.

The upshot is a Lagrangian which can be written in the standard form as the difference of a kinetic term and the Hamiltonian density:

$$
\mathscr{L}_{g f}=\mathscr{L}_{\text {kin }}-\mathcal{H}
$$

The kinetic term $\mathscr{L}_{\text {kin }}$ depends on the time derivatives of the physical fields, and determines the Poisson structure of the theory. It can be cast in the form

$$
\begin{aligned}
\mathscr{L}_{\text {kin }}=p_{M} \dot{x}_{M} & -\frac{\mathrm{i} P_{+}}{4} \operatorname{Str}\left(\Sigma_{+} \chi \partial_{\tau} \chi\right)+\frac{1}{2} g_{N} \pi_{M} \operatorname{Str}\left(\left[\Sigma_{N}, \Sigma_{M}\right] B_{\tau}\right) \\
& +\mathrm{i} \kappa \frac{\sqrt{\lambda}}{2}\left(G_{+}^{2}-G_{-}^{2}\right) \operatorname{Str}\left(F_{\tau} \widetilde{K}_{8} F_{\sigma}^{t} K_{8}\right)-\mathrm{i} \kappa \frac{\sqrt{\lambda}}{2} G_{M} G_{N} \operatorname{Str}\left(\Sigma_{N} F_{\tau} \Sigma_{M} \widetilde{K}_{8} F_{\sigma}^{t} K_{8}\right),
\end{aligned}
$$

where the functions $B_{\alpha}$ and $F_{\alpha}$ refer to the even and odd components of $g^{-1}(\chi) \partial_{\alpha} g(\chi)$ and are explicitly defined in appendix B.2 (equation (B.5)). As one can see, the kinetic term is highly nontrivial and leads to a complicated Poisson structure (similar to that derived in [32] for strings in $\mathfrak{s u}(1 \mid 1)$ subsector). To quantize the theory perturbatively, for example, in the near plane wave limit, we will need to redefine the fields so that the kinetic term would acquire the conventional form

$$
\mathscr{L}_{\text {kin }} \rightarrow p_{M} \dot{x}_{M}-\frac{\mathrm{i}}{2} \operatorname{Str}\left(\Sigma_{+} \chi \partial_{\tau} \chi\right)
$$


and, therefore, the redefined fields would satisfy the canonical commutation relations. This will be done in the next section up to the quartic order in the fields.

The density $\mathcal{H}$ of the Hamiltonian is given by the sum of $-\mathbf{p}_{-}$and a contribution of the Wess-Zumino term

$$
\begin{aligned}
& \mathcal{H}=-\mathbf{p}_{-}+\mathcal{H}_{\mathrm{WZ}} \\
& \begin{aligned}
\mathcal{H}_{\mathrm{WZ}}=\kappa \frac{\sqrt{\lambda}}{2}( & \left.G_{+}^{2}-G_{-}^{2}\right) \operatorname{Str}\left(\Sigma_{+} \chi \sqrt{1+\chi^{2}} \widetilde{K}_{8} F_{\sigma}^{t} K_{8}\right) \\
& +\kappa \frac{\sqrt{\lambda}}{2} G_{M} G_{N} \operatorname{Str}\left(\Sigma_{+} \Sigma_{N} \chi \sqrt{1+\chi^{2}} \Sigma_{M} \widetilde{K}_{8} F_{\sigma}^{t} K_{8}\right) .
\end{aligned}
\end{aligned}
$$

Here the explicit expression for $\mathbf{p}_{-}$is given by (B.4), and we also should use the formulae (B.16), (B.12) and (B.27) to express everything in terms of physical fields.

Let us stress that in this way we find the gauge-fixed Lagrangian as an exact function of the light-cone momentum $P_{+}$, and the string tension $\sqrt{\lambda}$. Then it is straightforward to consider various expansions of the Lagrangian; in particular, in the next sections we will consider a near plane wave expansion, with $P_{+} \rightarrow \infty$ with $\lambda / P_{+}^{2}$ fixed.

\section{Near plane wave expansion}

In this section, we discuss the near plane wave expansion of the gauge-fixed Lagrangian (4.1), which amounts to a large $P_{+}$limit with the effective coupling $\widetilde{\lambda}=\frac{4 \lambda}{P_{+}^{2}}$ fixed. For this we need to redefine the fields to reduce the kinetic term (4.2) to the canonical form (4.3) up to terms of sixth order in fields, and expand the density of the Hamiltonian to the quartic order. The resulting quartic Hamiltonian may be used to compute the $1 / J$ correction to the spacetime energy $E$ of an arbitrary string state.

\subsection{Field redefinition}

It is straightforward to find the necessary field redefinition to remove all quartic terms in the kinetic term of the Lagrangian. To this end, we note that the kinetic term can be written in the following form,

$$
\mathscr{L}_{\text {kin }}=p_{M} \dot{x}_{M}-\frac{\mathrm{i} P_{+}}{4} \operatorname{Str}\left(\Sigma_{+} \chi \dot{\chi}\right)+\frac{\mathrm{i} P_{+}}{2} \operatorname{Str}\left(\Sigma_{+} \Phi(p, x, \chi) \dot{\chi}\right),
$$

where $\Phi$ is a function of cubic order in physical fields and has the same structure as $\chi$, i.e. it satisfies the defining relations (3.7) for our $\kappa$-symmetry gauge. Then it is clear that the last term can be removed by the following redefinition of $\chi$ :

$$
\chi \rightarrow \chi+\Phi(p, x, \chi)
$$

This redefinition casts the kinetic term (5.1) into the form (up to a total derivative)

$$
\begin{aligned}
\mathscr{L}_{\text {kin }}=p_{M} \dot{x}_{M} & -\frac{\mathrm{i} P_{+}}{4} \operatorname{Str}\left(\Sigma_{+} \chi \dot{\chi}\right)+\frac{\mathrm{i} P_{+}}{2} \operatorname{Str}\left(\Sigma_{+}(\Phi(p, x, \chi+\Phi)-\Phi(p, x, \chi)) \dot{\chi}\right) \\
& +\frac{\mathrm{i} P_{+}}{4} \operatorname{Str}\left(\Sigma_{+} \Phi(p, x, \chi) \dot{\Phi}(p, x, \chi)\right) .
\end{aligned}
$$

Since $\Phi$ is at least of the cubic order in the fields, the terms on the second line of (5.3) are at least of the sixth order. These terms can also be removed by a similar field redefinition. However, this time one would need to redefine not only the fermions but the bosonic coordinates $x_{M}$ and $p_{M}$, too. For our purposes here it is sufficient to perform only the simplest redefinition 
(5.2), and just drop the terms on the second line of (5.3). This reduces the kinetic term to the canonical quadratic form which can be written in a very explicit form as

$$
\begin{aligned}
\mathscr{L}_{\text {kin }} & =p_{M} \dot{x}_{M}-\frac{\mathrm{i} P_{+}}{4} \operatorname{Str}\left(\Sigma_{+} \chi \dot{\chi}\right)=p_{M} \dot{x}_{M}+\frac{\mathrm{i} P_{+}}{4} \operatorname{tr}\left(\eta^{\dagger} \dot{\eta}+\theta^{\dagger} \dot{\theta}\right) \\
& =p_{M} \dot{x}_{M}+\frac{\mathrm{i} P_{+}}{2}\left(\eta_{a}^{\dagger} \dot{\eta}_{a}+\theta_{a}^{\dagger} \dot{\theta}_{a}\right) .
\end{aligned}
$$

Here we used the following decomposition of the fermions (see appendix A):

$\chi=\sigma_{+} \otimes \Theta+\sigma_{-} \otimes \Theta_{*}, \quad \Theta=\mathcal{P}_{+} \eta+\mathcal{P}_{-} \theta^{\dagger}, \quad \eta=\eta_{a} \Gamma_{a}, \quad \theta=\theta_{a} \Gamma_{a}$.

Rescaling the fermions

$$
\chi \rightarrow \sqrt{\frac{2}{P_{+}}} \chi, \quad \eta \rightarrow \sqrt{\frac{2}{P_{+}}} \eta, \quad \theta \rightarrow \sqrt{\frac{2}{P_{+}}} \theta,
$$

brings the kinetic term into the canonical form

$$
\mathscr{L}_{\text {kin }}=p_{M} \dot{x}_{M}+\mathrm{i} \eta_{a}^{\dagger} \dot{\eta}_{a}+\mathrm{i} \theta_{a}^{\dagger} \dot{\theta}_{a}
$$

which shows that $(p, x),\left(\eta^{\dagger}, \eta\right)$ and $\left(\theta^{\dagger}, \theta\right)$ are canonically conjugate pairs.

Before discussing the expansion of the Hamiltonian let us mention an important and nice property of the redefinition (5.2). One can check that up to the sixth order in fields, the formula (B.12) for $x_{-}^{\prime}$ takes the form

$$
x_{-}^{\prime}=-\frac{1}{P_{+}}\left(p_{M} x_{M}^{\prime}-\frac{\mathrm{i}}{4} P_{+} \operatorname{Str}\left(\Sigma_{+} \chi \chi^{\prime}\right)+\partial_{\sigma} f(p, x, \chi)\right),
$$

where $f(p, x, \chi)$ is a function of the momenta and coordinates. Thus, we see that integrating (5.8) over $\sigma$ we get the usual 'flat space' level-matching condition

$$
\mathcal{V}=\int_{0}^{2 \pi}\left(p_{M} x_{M}^{\prime}-\frac{\mathrm{i}}{4} P_{+} \operatorname{Str}\left(\Sigma_{+} \chi \chi^{\prime}\right)\right)=0
$$

which in terms of the rescaled fermions (5.6) takes the form

$$
\mathcal{V}=\int_{0}^{2 \pi}\left(p_{M} x_{M}^{\prime}+\mathrm{i} \eta_{a}^{\dagger} \eta_{a}^{\prime}+\mathrm{i} \theta_{a}^{\dagger} \theta_{a}^{\prime}\right)=0
$$

\subsection{Hamiltonian}

Here we derive the density $\mathcal{H}$ of the Hamiltonian up to the fourth order in fields. To this end we expand (4.4), and take into account the fermion redefinition (5.2). The fermion shift produces additional quartic terms in the Hamiltonian coming only from the shift of the quadratic part at this order.

The density of the complete quadratic Hamiltonian can be easily found by using formulae from appendix B:

$\mathcal{H}_{2}=\frac{1}{P_{+}} p_{M}^{2}+\frac{P_{+}}{4} x_{M}^{2}+\frac{\lambda}{P_{+}} x_{M}^{\prime 2}+\frac{\kappa}{2} \sqrt{\lambda} \operatorname{Str}\left(\Sigma_{+} \chi \widetilde{K}_{8} \chi^{\prime t} K_{8}\right)+\frac{P_{+}}{4} \operatorname{Str} \chi^{2}$.

We see from this equation and the kinetic term (5.4) that in order to have a canonical Poisson structure and a standard quadratic Hamiltonian of the form $\frac{1}{2} p_{M}^{2}+\frac{1}{2} x_{M}^{2}$ we should make the following rescaling of the fields:

$$
p_{M} \rightarrow \sqrt{\frac{P_{+}}{2}} p_{M}, \quad x_{M} \rightarrow \sqrt{\frac{2}{P_{+}}} x_{M}, \quad \chi \rightarrow \sqrt{\frac{2}{P_{+}}} \chi .
$$


Then the density of the quadratic Hamiltonian takes the form

$$
\mathcal{H}_{2}=\frac{1}{2} p_{M}^{2}+\frac{1}{2} x_{M}^{2}+\frac{\tilde{\lambda}}{2} x_{M}^{\prime 2}+\frac{\kappa}{2} \sqrt{\tilde{\lambda}} \operatorname{Str}\left(\Sigma_{+} \chi \widetilde{K}_{8} \chi^{\prime t} K_{8}\right)+\frac{1}{2} \operatorname{Str} \chi^{2},
$$

where

$$
\tilde{\lambda}=\frac{4 \lambda}{P_{+}^{2}}
$$

is the effective coupling constant which is kept finite in the plane wave limit $P_{+} \rightarrow \infty$ or equivalently $\lambda \rightarrow \infty$. This limit is the light-cone gauge equivalent of the usual $J \rightarrow \infty \mathrm{BMN}$ limit. Note that the coupling $\tilde{\lambda}$ is not equal to the effective coupling $\lambda^{\prime}=\frac{\lambda}{J^{2}}$ but reduces to it only in the strict $J \rightarrow \infty$ limit.

In terms of the rescaled bosons and fermions $\eta$ and $\theta \mathcal{H}_{2}$ takes the form

$\mathcal{H}_{2}=\frac{p_{M}^{2}}{2}+\frac{x_{M}^{2}}{2}+\frac{\tilde{\lambda}}{2} x_{M}^{\prime 2}+\frac{1}{2} \operatorname{tr}\left(\eta^{\dagger} \eta+\theta^{\dagger} \theta+\frac{\kappa \sqrt{\tilde{\lambda}}}{2}\left(\eta \eta^{\prime}+\theta \theta^{\prime}-\eta^{\dagger} \eta^{\prime \dagger}-\theta^{\dagger} \theta^{\prime \dagger}\right)\right)$.

The quartic Hamiltonian is also straightforwardly derived. The details of the computation can be found in appendices $C$ and D. In terms of the rescaled fields (5.12) it takes the form

$$
\begin{aligned}
\mathcal{H}_{4}=\frac{1}{2 P_{+}}[ & 2 \tilde{\lambda}\left(y^{\prime 2} z^{2}-z^{\prime 2} y^{2}+z^{\prime 2} z^{2}-y^{\prime 2} y^{2}\right)-\tilde{\lambda} \operatorname{Str}\left(\frac{1}{2} \chi \chi^{\prime} \chi \chi^{\prime}+\chi^{2} \chi^{\prime 2}\right. \\
& \left.+\frac{1}{4}\left(\chi \chi^{\prime}-\chi^{\prime} \chi\right) K_{8}\left(\chi \chi^{\prime}-\chi^{\prime} \chi\right)^{t} K_{8}+\chi \widetilde{K}_{8} \chi^{\prime t} K_{8} \chi \widetilde{K}_{8} \chi^{\prime t} K_{8}\right) \\
& +\tilde{\lambda} \operatorname{Str}\left(\left(z^{2}-y^{2}\right) \chi^{\prime} \chi^{\prime}+\frac{1}{2} x_{M}^{\prime} x_{N}\left[\Sigma_{M}, \Sigma_{N}\right]\left(\chi \chi^{\prime}-\chi^{\prime} \chi\right)-2 x_{M} x_{N} \Sigma_{M} \chi^{\prime} \Sigma_{N} \chi^{\prime}\right) \\
& \left.+\frac{\mathrm{i} \kappa \sqrt{\lambda}}{4}\left(x_{N} p_{M}\right)^{\prime} \operatorname{Str}\left(\left[\Sigma_{N}, \Sigma_{M}\right]\left(\widetilde{K}_{8} \chi^{t} K_{8} \chi-\chi \widetilde{K}_{8} \chi^{t} K_{8}\right)\right)\right] .
\end{aligned}
$$

One can easily see that the uniform light-cone gauge quartic Hamiltonian (5.16) is considerably simpler than the quartic Hamiltonian obtained by Calan et al [19], though still rather involved. An important property of the Hamiltonian is that it vanishes in the point-particle limit, when all fields do not depend on $\sigma$. One can show that the same property is also valid for the sixth-order Hamiltonian. This is in accord with the observation that already the quadratic particle Hamiltonian reproduces the spectrum of type IIB supergravity on $\operatorname{AdS}_{5} \times \mathrm{S}^{5}$.

It is convenient to express the Hamiltonian in terms of the (rescaled) complex bosonic fields $Z_{a}, Y_{a}$ (see also appendix A),

$$
\begin{aligned}
& Z_{1}=z_{2}+\mathrm{i} z_{1}, \quad Z_{2}=z_{4}+\mathrm{i} z_{3}, \quad Z_{4}=z_{2}-\mathrm{i} z_{1}, \quad Z_{3}=z_{4}-\mathrm{i} z_{3}, \\
& Y_{1}=y_{2}+\mathrm{i} y_{1}, \quad Y_{2}=y_{4}+\mathrm{i} y_{3}, \quad Y_{4}=y_{2}-\mathrm{i} y_{1}, \quad Y_{3}=y_{4}-\mathrm{i} y_{3},
\end{aligned}
$$

and their canonical momenta (associated with $z_{a}$ or $y_{a}$ )

$P_{1}=\frac{1}{2}\left(p_{2}+\mathrm{i} p_{1}\right), \quad P_{4}=\frac{1}{2}\left(p_{2}-\mathrm{i} p_{1}\right), \quad P_{2}=\frac{1}{2}\left(p_{4}+\mathrm{i} p_{3}\right), \quad P_{3}=\frac{1}{2}\left(p_{4}-\mathrm{i} p_{3}\right)$,

with

$$
Z_{a}^{\dagger}=Z_{5-a}, \quad Y_{a}^{\dagger}=Y_{5-a}, \quad P_{a}^{z \dagger}=P_{5-a}^{z}, \quad P_{a}^{y \dagger}=P_{5-a}^{y} .
$$

The convention is chosen such that $p_{M} x_{M}=P_{5-a}^{z} Z_{a}+P_{5-a}^{y} Y_{a}$. Then the kinetic term (5.7) takes the form

$$
\mathscr{L}_{\text {kin }}=P_{5-a}^{z} \dot{Z}_{a}+P_{5-a}^{y} \dot{Y}_{a}+\mathrm{i} \eta_{a}^{\dagger} \dot{\eta}_{a}+\mathrm{i} \theta_{a}^{\dagger} \dot{\theta}_{a} \quad \text { with } \quad a=1,2,3,4 .
$$


In terms of the complex fields the quadratic Hamiltonian acquires the form

$$
\begin{aligned}
\mathcal{H}_{2}=P_{5-a}^{z} P_{a}^{z} & +P_{5-a}^{y} P_{a}^{y}+\frac{1}{4}\left(Z_{5-a} Z_{a}+Y_{5-a} Y_{a}\right)+\frac{\tilde{\lambda}}{4}\left(Z_{5-a}^{\prime} Z_{a}^{\prime}+Y_{5-a}^{\prime} Y_{a}^{\prime}\right) \\
& +\frac{1}{2} \operatorname{tr}\left(\eta^{\dagger} \eta+\theta^{\dagger} \theta+\frac{\kappa \sqrt{\lambda}}{2}\left(\eta \eta^{\prime}+\theta \theta^{\prime}-\eta^{\dagger} \eta^{\prime \dagger}-\theta^{\dagger} \theta^{\prime \dagger}\right)\right)
\end{aligned}
$$

and the quartic Hamiltonian is given by the following sum,

$$
\mathcal{H}_{4}=\mathcal{H}_{b b}+\mathcal{H}_{b f}+\mathcal{H}_{f f}
$$

where

$$
\begin{aligned}
& \mathcal{H}_{b b}=\frac{\tilde{\lambda}}{4 P_{+}}\left(Y_{5-a}^{\prime} Y_{a}^{\prime} Z_{5-b} Z_{b}-Y_{5-a} Y_{a} Z_{5-b}^{\prime} Z_{b}^{\prime}+Z_{5-a}^{\prime} Z_{a}^{\prime} Z_{5-b} Z_{b}-Y_{5-a}^{\prime} Y_{a}^{\prime} Y_{5-b} Y_{b}\right) \\
& \mathcal{H}_{b f}=\frac{1}{2 P_{+}} \operatorname{tr}\left[\frac{\tilde{\lambda}}{2}\left(Z_{5-a} Z_{a}-Y_{5-a} Y_{a}\right)\left(\eta^{\prime \dagger} \eta^{\prime}+\theta^{\prime \dagger} \theta^{\prime}\right)\right. \\
&-\frac{\tilde{\lambda}}{2} Z_{m}^{\prime} Z_{n}\left[\Gamma_{m}, \Gamma_{n}\right]\left(\mathcal{P}_{+}\left(\eta \eta^{\prime \dagger}-\eta^{\prime} \eta^{\dagger}\right)-\mathcal{P}_{-}\left(\theta^{\dagger} \theta^{\prime}-\theta^{\prime \dagger} \theta\right)\right. \\
&+\frac{\tilde{\lambda}}{2} Y_{m}^{\prime} Y_{n}\left[\Gamma_{m}, \Gamma_{n}\right]\left(-\mathcal{P}_{-}\left(\eta^{\dagger} \eta^{\prime}-\eta^{\prime \dagger} \eta\right)+\mathcal{P}_{+}\left(\theta \theta^{\prime \dagger}-\theta^{\prime} \theta^{\dagger}\right)\right) \\
&-\frac{\mathrm{i} \kappa}{2} \sqrt{\tilde{\lambda}}\left(Z_{n} P_{m}^{z}\right)^{\prime}\left[\Gamma_{n}, \Gamma_{m}\right]\left(\mathcal{P}_{+}\left(\eta^{\dagger} \eta^{\dagger}+\eta \eta\right)+\mathcal{P}_{-}\left(\theta^{\dagger} \theta^{\dagger}+\theta \theta\right)\right) \\
&+\frac{\mathrm{i} \kappa}{2} \sqrt{\tilde{\lambda}}\left(Y_{n} P_{m}^{y}\right)^{\prime}\left[\Gamma_{n}, \Gamma_{m}\right]\left(\mathcal{P}_{-}\left(\eta^{\dagger} \eta^{\dagger}+\eta \eta\right)+\mathcal{P}_{+}\left(\theta^{\dagger} \theta^{\dagger}+\theta \theta\right)\right) \\
&\left.+4 \tilde{i} \tilde{\lambda} Z_{m} Y_{n}\left(-\mathcal{P}_{-} \Gamma_{m} \eta^{\prime} \Gamma_{n} \theta^{\prime}+\mathcal{P}_{+} \Gamma_{m} \theta^{\prime \dagger} \Gamma_{n} \eta^{\prime \dagger}\right)\right]
\end{aligned}
$$

The quartic fermionic term can be written in the form

$$
\mathcal{H}_{f f}=\mathcal{H}_{f f}(\eta)-\mathcal{H}_{f f}(\theta),
$$

where $H_{f f}(\eta)$ takes the following amazingly simple form:

$$
\mathcal{H}_{f f}(\eta)=-\frac{\tilde{\lambda}}{4 P_{+}} \operatorname{tr} \Sigma\left(\eta^{\prime \dagger} \eta \eta^{\prime \dagger} \eta+\eta^{\dagger} \eta^{\prime} \eta^{\dagger} \eta^{\prime}+\eta^{\prime \dagger} \eta^{\dagger} \eta^{\prime \dagger} \eta^{\dagger}+\eta^{\prime} \eta \eta^{\prime} \eta\right) .
$$

\section{Quantization}

We now turn to the perturbative quantization of the light-cone $\mathrm{AdS}_{5} \times \mathrm{S}^{5}$ superstring in the near plane wave limit. Due to the fermionic field redefinitions performed above up to the sixth order the kinetic Lagrangian is of canonical form. Promoting all fields to operators, we read off from (5.19) the (anti)commutation relations

$$
\left[Z_{a}, P_{5-b}^{z}\right]=\mathrm{i} \delta_{a b} \quad\left[Y_{a}, P_{5-b}^{y}\right]=\mathrm{i} \delta_{a b} \quad\left\{\eta_{a}, \eta_{b}^{\dagger}\right\}=\delta_{a b} \quad\left\{\theta_{a}, \theta_{b}^{\dagger}\right\}=\delta_{a b} .
$$

We now need to establish a mode decomposition of the bosonic and fermionic fields which renders the quadratic piece of the Hamiltonian (5.20) in a diagonal form. This will be done for the bosonic and fermionic sector in the following. 


\subsection{Representation for bosons}

The bosonic part of the quadratic Hamiltonian (5.20) has the form

$H_{\mathrm{bos}}^{(0)}=P_{5-a}^{z} P_{a}^{z}+P_{5-a}^{y} P_{a}^{y}+\frac{1}{4}\left(Z_{5-a} Z_{a}+Y_{5-a} Y_{a}\right)+\frac{\tilde{\lambda}}{4}\left(Z_{5-a}^{\prime} Z_{a}^{\prime}+Y_{5-a}^{\prime} Y_{a}^{\prime}\right)$.

We shall choose the following mode decompositions for $Z_{a}$ and $P_{a}^{z}$,

$$
\begin{array}{ll}
Z_{a}(\tau, \sigma)=\sum_{n} \mathrm{e}^{\mathrm{i} n \sigma} Z_{a, n}(\tau), & Z_{a, n}^{\dagger}(\tau, \sigma)=Z_{5-a,-n}(\tau . \sigma) ; \\
P_{a}^{z}(\tau, \sigma)=\sum_{n} \mathrm{e}^{\mathrm{i} n \sigma} P_{a, n}^{z}(\tau), & P_{a, n}^{z \dagger}(\tau, \sigma)=P_{5-a,-n}^{z}(\tau, \sigma), \\
P_{a, n}^{z}=\frac{\sqrt{\omega_{n}}}{2}\left(\beta_{a, n}^{+}+\beta_{5-a,-n}^{-}\right), & Z_{a, n}=\frac{1}{\mathrm{i} \sqrt{\omega_{n}}}\left(\beta_{a, n}^{+}-\beta_{5-a,-n}^{-}\right),
\end{array}
$$

and similarly for $Y_{a}$ and $P_{a}^{y}$

$$
\begin{array}{ll}
Y_{a}(\tau, \sigma)=\sum_{n} \mathrm{e}^{\mathrm{i} n \sigma} Y_{a, n}(\tau), & Y_{a, n}^{\dagger}(\tau, \sigma)=Y_{5-a,-n}(\tau, \sigma) ; \\
P_{a}^{y}(\tau, \sigma)=\sum_{n} \mathrm{e}^{\mathrm{i} n \sigma} P_{a, n}^{y}(\tau), & P_{a, n}^{y \dagger}(\tau, \sigma)=P_{5-a,-n}^{y}(\tau, \sigma), \\
P_{a, n}^{y}=\frac{\sqrt{\omega_{n}}}{2}\left(\alpha_{a, n}^{+}+\alpha_{5-a,-n}^{-}\right), & Y_{a, n}=\frac{1}{\mathrm{i} \sqrt{\omega_{n}}}\left(\alpha_{a, n}^{+}-\alpha_{5-a,-n}^{-}\right),
\end{array}
$$

where the frequency $\omega_{n}$ is defined as

$$
\omega_{n}=\sqrt{1+\tilde{\lambda} n^{2}}
$$

Then in terms of the creation and annihilation operators the quadratic bosonic Lagrangian, stemming from the kinetic piece (5.19) and Hamiltonian (5.11), takes the form

$$
L_{\mathrm{bos}}^{(0)}=\mathrm{i} \sum_{a, n}\left(\alpha_{a, n}^{+} \dot{\alpha}_{a, n}^{-}+\beta_{a, n}^{+} \dot{\beta}_{a, n}^{-}\right)-\sum_{a, n} \omega_{n}\left(\alpha_{a, n}^{+} \alpha_{a, n}^{-}+\beta_{a, n}^{+} \beta_{a, n}^{-}\right) .
$$

This shows that in quantum theory the only nontrivial commutators are simply

$$
\left[\alpha_{a, n}^{-}, \alpha_{a, n}^{+}\right]=1, \quad\left[\beta_{a, n}^{-}, \beta_{a, n}^{+}\right]=1,
$$

and we have the standard quadratic Hamiltonian.

\subsection{Representation for fermions}

For the quadratic fermionic sector we have the Hamiltonian from (5.20)

$$
H_{\mathrm{ferm}}^{(0)}=\frac{1}{2} \operatorname{tr}\left(\eta^{\dagger} \eta+\theta^{\dagger} \theta-\frac{\kappa}{2} \sqrt{\tilde{\lambda}}\left(-\eta \eta^{\prime}-\theta \theta^{\prime}+\eta^{\dagger} \eta^{\prime \dagger}+\theta^{\dagger} \theta^{\prime \dagger}\right)\right) .
$$

Our mode decomposition for fermions follows a similar construction found in [32] and reads

$$
\begin{array}{ll}
\eta(\tau, \sigma)=\sum_{n} \mathrm{e}^{\mathrm{i} n \sigma} \eta_{n}(\tau), & \eta^{\dagger}(\tau, \sigma)=\sum_{n} \mathrm{e}^{-\mathrm{i} n \sigma} \eta_{n}^{\dagger}(\tau), \\
\theta(\tau, \sigma)=\sum_{n} \mathrm{e}^{\mathrm{i} n \sigma} \theta_{n}(\tau), & \theta^{\dagger}(\tau, \sigma)=\sum_{n} \mathrm{e}^{-\mathrm{i} n \sigma} \theta_{n}^{\dagger}(\tau) \\
\eta_{n}=f_{n} \eta_{-n}^{-}+\mathrm{i} g_{n} \eta_{n}^{+}, & \eta_{n}^{\dagger}=f_{n} \eta_{-n}^{+}-\mathrm{i} g_{n} \eta_{n}^{-} . \\
\theta_{n}=f_{n} \theta_{-n}^{-}+\mathrm{i} g_{n} \theta_{n}^{+}, & \theta_{n}^{\dagger}=f_{n} \theta_{-n}^{+}-\mathrm{i} g_{n} \theta_{n}^{-} .
\end{array}
$$


Here we have introduced the quantities

$$
f_{n}=\sqrt{\frac{1}{2}\left(1+\frac{1}{\omega_{n}}\right)}, \quad g_{n}=\frac{\kappa \sqrt{\tilde{\lambda} n}}{1+\omega_{n}} f_{n} .
$$

Note that $g_{n}^{2}=\frac{1}{2}\left(1-\frac{1}{\omega_{n}}\right)$. In terms of the creation and annihilation operators the quadratic fermion Lagrangian then indeed takes the diagonalized form

$$
L_{\text {ferm }}^{(0)}=\frac{\mathrm{i}}{2} \operatorname{tr} \sum_{n}\left(\eta_{n}^{+} \dot{\eta}_{n}^{-}+\theta_{n}^{+} \dot{\theta}_{n}^{-}\right)-\frac{1}{2} \operatorname{tr} \sum_{n} \omega_{n}\left(\eta_{n}^{+} \eta_{n}^{-}+\theta_{n}^{+} \theta_{n}^{-}\right) .
$$

If, furthermore, we use the decomposition of the matrices $\eta$ and $\theta$ in terms of the Dirac matrices of appendix $\mathrm{A}$ as

$$
\eta_{n}^{-}=\eta_{a, n}^{-} \Gamma_{5-a}, \quad \eta_{n}^{+}=\eta_{a, n}^{+} \Gamma_{a}, \quad \theta_{n}^{-}=\theta_{a, n}^{-} \Gamma_{5-a}, \quad \theta_{n}^{+}=\theta_{a, n}^{+} \Gamma_{a},
$$

and the identity $\operatorname{tr} \Gamma_{a} \Gamma_{5-b}=2 \delta_{a b}$, then the quadratic Lagrangian may be rewritten as

$$
L_{\text {ferm }}^{(0)}=\mathrm{i} \sum_{n}\left(\eta_{a, n}^{+} \dot{\eta}_{a, n}^{-}+\theta_{a, n}^{+} \dot{\theta}_{a, n}^{-}\right)-\sum_{n} \omega_{n}\left(\eta_{a, n}^{+} \eta_{a, n}^{-}+\theta_{a, n}^{+} \theta_{a, n}^{-}\right) .
$$

This shows that in quantum theory the only nontrivial anticommutators between fermionic mode operators are

$$
\left\{\eta_{a, n}^{-}, \eta_{a, n}^{+}\right\}=1, \quad\left\{\theta_{a, n}^{-}, \theta_{a, n}^{+}\right\}=1,
$$

and we have a standard diagonal quadratic Hamiltonian in the fermionic sector as well.

Note that we will take the quartic Hamiltonian $H_{b b}+H_{b f}+H_{f f}$ to be normal-ordered with respect to these bosonic and fermionic oscillator modes.

\subsection{Generic string state}

The generic eigenstate of the quadratic Hamiltonian can now be written in the form

$$
|\Psi\rangle=\prod_{c=1}^{4}\left|\theta_{c}, M_{\theta}^{c}\right\rangle \otimes \prod_{c=1}^{4}\left|\eta_{c}, M_{\eta}^{c}\right\rangle \otimes \prod_{c=1}^{4}\left|Z_{c}, M_{z}^{c}\right\rangle \otimes \prod_{c=1}^{4}\left|Y_{c}, M_{y}^{c}\right\rangle,
$$

where we assume the products to be in the decreasing order $\prod_{c=1}^{4} f_{c} \equiv f_{4} f_{3} f_{2} f_{1}$, and take

$$
\begin{aligned}
\left|\theta_{c}, M_{\theta}^{c}\right\rangle & \equiv \theta_{c}^{+}\left(n_{M_{\theta}^{c}}\right) \theta_{c}^{+}\left(n_{M_{\theta}^{c}-1}\right) \cdots \theta_{c}^{+}\left(n_{2}\right) \theta_{c}^{+}\left(n_{1}\right)|0\rangle, \\
\left|\eta_{c}, M_{\eta}^{c}\right\rangle & \equiv \eta_{c}^{+}\left(m_{M_{\eta}^{c}}\right) \eta_{c}^{+}\left(m_{M_{\eta}^{c}-1}\right) \cdots \eta_{c}^{+}\left(m_{2}\right) \eta_{c}^{+}\left(m_{1}\right)|0\rangle, \\
\left|Z_{c}, M_{z}^{c}\right\rangle & \equiv \beta_{c}^{+}\left(l_{M_{z}^{c}}\right) \beta_{c}^{+}\left(l_{M_{z}^{c}-1}\right) \cdots \beta_{c}^{+}\left(l_{2}\right) \beta_{c}^{+}\left(l_{1}\right)|0\rangle, \\
\left|Y_{c}, M_{y}^{c}\right\rangle & \equiv \alpha_{c}^{+}\left(k_{M_{y}^{c}}\right) \alpha_{c}^{+}\left(k_{M_{y}^{c}-1}\right) \cdots \alpha_{c}^{+}\left(k_{2}\right) \alpha_{c}^{+}\left(k_{1}\right)|0\rangle .
\end{aligned}
$$

In the above, we have used the notation $\eta_{c}^{+}(m) \equiv \eta_{c, m}^{+}$and so on, and we assumed that the mode numbers form increasing sequences, i.e. for fermions

$$
n_{1}<n_{2}<\cdots<n_{M_{\theta}^{c}-1}<n_{M_{\theta}^{c}}, \quad m_{1}<m_{2}<\cdots<m_{M_{\eta}^{c}-1}<m_{M_{\eta}^{c}}
$$

and for bosons

$$
l_{1} \leqslant l_{2} \leqslant \cdots \leqslant l_{M_{z}^{c}-1} \leqslant l_{M_{z}^{c}}, \quad k_{1} \leqslant k_{2} \leqslant \cdots \leqslant k_{M_{y}^{c}-1} \leqslant k_{M_{y}^{c}} .
$$

The energy of this state is

$$
H_{2}|\Psi\rangle=E|\Psi\rangle, \quad E=\sum_{\text {mode numbers }} \omega_{\text {mode number }}
$$


The string states must also satisfy the level-matching condition (5.9) that in terms of the creation and annihilation operators takes the form

$$
\mathcal{V}=\sum_{a, n} n\left(\alpha_{a, n}^{+} \alpha_{a, n}^{-}+\beta_{a, n}^{+} \beta_{a, n}^{-}+\eta_{a, n}^{+} \eta_{a, n}^{-}+\theta_{a, n}^{+} \theta_{a, n}^{-}\right)
$$

It just states that the sum of all mode numbers vanishes

$$
\mathcal{V}|\Psi\rangle=0 \Rightarrow \sum_{\text {all mode numbers }} \text { (mode number) }=0 \text {. }
$$

\section{Sectors and $1 / J$ correction}

It is known that in $\mathcal{N}=4 \mathrm{SYM}$ there are sectors of operators closed under the action of the dilatation operator; see [9] and references therein. In this section, we explain how string states dual to operators from $\mathfrak{s u}(2), \mathfrak{s l}(2), \mathfrak{s u}(1 \mid 1), \mathfrak{s u}(1 \mid 2)$ and $\mathfrak{s u}(2 \mid 3)$ sectors can be constructed starting from corresponding eigenstates of the quadratic Hamiltonian, and compute $1 / J$ corrections to energies of the states in the $\mathfrak{s u}(2), \mathfrak{s l}(2), \mathfrak{s u}(1 \mid 1)$ sectors.

\section{1. $\mathfrak{s u}(2)$ sector}

The $\mathfrak{s u}(2)$ sector of $\mathcal{N}=4 \mathrm{SYM}$ consists of operators of the form

$$
O_{s u(2)}=\operatorname{tr}\left(Z^{J} X^{M}+\text { permutations }\right),
$$

where $Z$ and $X$ are the two complex scalars carrying unit charges under the two $U$ (1) subgroups of $S U$ (4) that in the string picture correspond to the $U(1)$ generating shifts of the angle $\phi$ of $S^{5}$ and the $U(1)$ generated by $\Phi_{1}^{S}$, respectively; see appendix A. The operators, correspondingly, carry $J$ and $M$ units of charges. They are highest weight which means that they have minimal conformal dimensions among all the operators with the given charges.

Dual string states can be easily identified in the BMN limit $P_{+} \rightarrow \infty, \tilde{\lambda}$ fixed. First of all the charge $J$ is assigned to the light-cone vacuum and no creation and annihilation operator carries charges under this $U(1)$. Then from the tables of charges in appendix A we see that the string state carrying $M$ units of charge under the second $U(1)$ and having the minimal energy is obtained by acting on the vacuum by $M$ creation operators $\alpha_{1, n}^{+}$. Therefore, in the BMN limit the string states dual to operators from the $\mathfrak{s u}(2)$ sector are the states

$$
\left|\Psi_{s u(2)}\right\rangle=\alpha_{1, n_{M}}^{+} \alpha_{1, n_{M-1}}^{+} \cdots \alpha_{1, n_{1}}^{+}|0\rangle,
$$

which are eigenstates of the quadratic Hamiltonian with the energy

$$
E_{0}=\sum_{k=1}^{M} \omega_{n_{k}}
$$

and satisfy the level-matching condition

$$
\sum_{k=1}^{M} n_{k}=0
$$

For generic values of the mode numbers $n_{k}$ there is no nontrivial degeneracy in the spectrum ${ }^{11}$, and the leading $1 / P_{+}$correction to the energy of the string state can be found just by computing

\footnotetext{
${ }^{11}$ Given a state with mode numbers $\left\{n_{k}\right\}$, the state with the mode numbers $\left\{-n_{k}\right\}$ has the same energy. One can easily see that the states do not mix with each other. One can also have a situation when mode numbers are divided into several groups, each group satisfying the level-matching condition. Then changing the signs of the mode numbers in any of the groups leads to a state with the same energy. One can show that these states do not mix at least at the $1 / P_{+}$ order.
} 
the average of the quartic Hamiltonian $H_{b b}$ in the state (7.2). The computation is very simple because there is only one term in $H_{b b}$ contributing to the average. Explicitly, we find

$$
\begin{aligned}
\left\langle\Psi_{s u(2)}\left|H_{b b}\right| \Psi_{s u(2)}\right\rangle & =-\frac{\tilde{\lambda}}{4 P_{+}} \int_{0}^{2 \pi} \frac{\mathrm{d} \sigma}{2 \pi}\left\langle\Psi_{s u(2)}\left|Y_{a}^{\prime} Y_{5-a}^{\prime} Y_{b} Y_{5-b}\right| \Psi_{s u(2)}\right\rangle \\
& =\frac{\tilde{\lambda}}{4 P_{+}} \sum_{n+m+k+l=0} \frac{n m}{\sqrt{\omega_{n} \omega_{m} \omega_{k} \omega_{l}}}\left\langle\Psi_{s u(2)}\left|4 \alpha_{a, n}^{+} \alpha_{b, k}^{+} \alpha_{a,-m}^{-} \alpha_{b,-l}^{-}\right| \Psi_{s u(2)}\right\rangle \\
& =-\frac{\tilde{\lambda}}{P_{+}} \sum_{k \neq j}^{M} \frac{n_{j} n_{k}+n_{k}^{2}}{\omega_{j} \omega_{k}}=-\frac{\tilde{\lambda}}{2 P_{+}} \sum_{k \neq j}^{M} \frac{\left(n_{j}+n_{k}\right)^{2}}{\omega_{j} \omega_{k}},
\end{aligned}
$$

where for simplicity we used the notation $\omega_{j} \equiv \omega_{n_{j}}=\sqrt{1+\tilde{\lambda} n_{k}^{2}}$. Now to find the $1 / J$ correction to the energy of the string state we should solve the equation

$$
E-J=\sum_{k=1}^{M} \sqrt{1+\frac{4 \lambda n_{k}^{2}}{(E+J)^{2}}}-\frac{\tilde{\lambda}}{2 P_{+}} \sum_{k \neq j}^{M} \frac{\left(n_{j}+n_{k}\right)^{2}}{\omega_{j} \omega_{k}}
$$

in powers of $1 / J$ keeping $\lambda^{\prime}=\lambda / J^{2}$ and $M$ finite. A simple algebra gives

$$
E_{s u(2)}-J=\sum_{k=1}^{M} \bar{\omega}_{k}-\frac{\lambda^{\prime}}{4 J} \sum_{k=1}^{M} \sum_{j=1}^{M} \frac{n_{k}^{2} \bar{\omega}_{j}^{2}+n_{j}^{2} \bar{\omega}_{k}^{2}}{\bar{\omega}_{k} \bar{\omega}_{j}}-\frac{\lambda^{\prime}}{4 J} \sum_{k \neq j}^{M} \frac{\left(n_{j}+n_{k}\right)^{2}}{\bar{\omega}_{j} \bar{\omega}_{k}}
$$

where now $\bar{\omega}_{k}:=\sqrt{1+\lambda^{\prime} n_{k}^{2}}$ with the BMN coupling constant $\lambda^{\prime}:=\lambda / J^{2}$. Taking into account the level-matching condition one can easily check that (7.6) coincides with the expression obtained in [24] by using the quantum string Bethe ansatz, and in [42] by using a rather complicated string Hamiltonian in the uniform gauge $t=\tau, p_{\phi}=J$.

Since the quartic Hamiltonian $H_{4}$ contains terms describing interactions of operators $\alpha_{1, n}^{ \pm}$with operators charged under other $U(1)$ subgroups, the state (7.2) gets corrections which depend on these operators. We will argue at the end of this section that there is a unitary transformation which transforms the Hamiltonian to such a form that the action of the transformed Hamiltonian on the states (7.2) (and in general on states dual to operators from closed sectors) is closed. This Hamiltonian is a string analogue of the field theory dilatation operator, and its restriction to operators $\alpha_{1, n}^{ \pm}$can be considered as an effective Hamiltonian for the $\mathfrak{s u}(2)$ sector.

\section{2. $\mathfrak{s l}(2)$ sector}

The $\mathfrak{s l}(2)$ sector of $\mathcal{N}=4$ SYM consists of operators of the form

$$
O_{s l(2)}=\operatorname{tr}\left(D_{-}^{M} Z^{J}+\text { permutations }\right)
$$

where $D_{-}$is the covariant derivative in a light-cone direction carrying unit charge under the $U$ (1) subgroup of $S U(2,2)$, that in the string picture corresponds to the $U(1)$ generated by $\Phi_{1}^{\text {AdS }}$; see appendix A. The operators, correspondingly, carry $J$ and $M$ units of the charges. They are again highest weight, and string states dual to operators from the $\mathfrak{s l}(2)$ sector of $\mathcal{N}=4$ SYM are easily identified by analysing the tables of charges in appendix A. We see that the string state carrying $M$ units of the charge $S_{1}$ and having the minimal energy is obtained by acting on the vacuum by $M$ creation operators $\beta_{1, n}^{+}$. Therefore, in the BMN limit the string states dual to operators from the $\mathfrak{s l}(2)$ sector are the states

$$
\left|\Psi_{s l(2)}\right\rangle=\beta_{1, n_{M}}^{+} \beta_{1, n_{M-1}}^{+} \cdots \beta_{1, n_{1}}^{+}|0\rangle .
$$


The computation of the $1 / P_{+}$correction to this state literally repeats the computation we did for the $\mathfrak{s u}(2)$ sector. The only change is the opposite sign of the correction (7.4), compare (5.22), i.e. we have

$$
E-J=\sum_{k=1}^{M} \sqrt{1+\frac{4 \lambda n_{k}^{2}}{(E+J)^{2}}}+\frac{\tilde{\lambda}}{2 P_{+}} \sum_{k \neq j}^{M} \frac{\left(n_{j}+n_{k}\right)^{2}}{\omega_{j} \omega_{k}}
$$

and therefore, the $1 / J$ correction for the $\mathfrak{s l}(2)$ state takes the form

$E_{s l(2)}-J=\sum_{k=1}^{M} \bar{\omega}_{k}-\frac{\lambda^{\prime}}{4 J} \sum_{k=1}^{M} \sum_{j=1}^{M} \frac{n_{k}^{2} \bar{\omega}_{j}^{2}+n_{j}^{2} \bar{\omega}_{k}^{2}}{\bar{\omega}_{k} \bar{\omega}_{j}}+\frac{\lambda^{\prime}}{4 J} \sum_{k \neq j}^{M} \frac{\left(n_{j}+n_{k}\right)^{2}}{\bar{\omega}_{j} \bar{\omega}_{k}}$.

Again it is straightforward to check that (7.10) coincides with the expression obtained in [42]. Let us mention that the fact that the $1 / J$ correction for $\mathfrak{s u}(2)$ states differs from the one for $\mathfrak{s l}(2)$ states just by a sign of one term seems not to have been noticed before. This sign difference between the $\mathfrak{s u}(2)$ and $\mathfrak{s l}(2)$ reflects the fact that curvatures in the $S^{5}$ and $A d S_{5}$ parts are equal but of opposite signs.

\section{3. $\mathfrak{s u}(1 \mid 1)$ sector}

The detailed discussion of the $\mathfrak{s u}(1 \mid 1)$ sector in $\mathcal{N}=4$ SYM and string theory was given in $[28,32,43]$. We find it useful, for completeness and since we have changed the basis of gamma matrices, to review shortly the consideration in [32].

The $\mathfrak{s u}(1 \mid 1)$ sector of $\mathcal{N}=4$ SYM consists of operators of the form

$$
O_{s u(1 \mid 1)}=\operatorname{tr}\left(Z^{J-\frac{M}{2}} \Psi^{M}+\text { permutations }\right) \text {. }
$$

The fermion $\Psi$ is the highest weight component of the gaugino from the vector multiplet. The gaugino $\Psi_{\alpha}$ belongs to the vector multiplet, it is neutral under $\mathfrak{s u}(3)$ which rotates the three complex scalars between themselves, and it carries the same charge $1 / 2$ under any of the three $U(1)$ subgroups of $S U(4)$. The corresponding Lie algebra element is

$$
\Phi_{\underline{\mathfrak{u}(1)} \times \mathfrak{s u}(3)}=\left(\begin{array}{cccc}
\mathrm{i}\left(\xi_{1}+\xi_{2}+\xi_{3}\right) & 0 & 0 & 0 \\
0 & -\mathrm{i} \xi_{1} & \alpha_{1}+\mathrm{i} \beta_{1} & \alpha_{2}+\mathrm{i} \beta_{2} \\
0 & -\alpha_{1}+\mathrm{i} \beta_{1} & -\mathrm{i} \xi_{2} & \alpha_{4}+\mathrm{i} \beta_{4} \\
0 & -\alpha_{2}+\mathrm{i} \beta_{2} & -\alpha_{4}+\mathrm{i} \beta_{4} & -\mathrm{i} \xi_{3}
\end{array}\right),
$$

where the $\mathfrak{s u}(3)$ part is obviously specified by choosing $\xi_{3}=-\xi_{1}-\xi_{2}$. It also transforms as a spinor under one of the $\mathfrak{s u}(2)$ from the Lorentz algebra $\mathfrak{s u}(2,2)$ and is neutral under the other. Therefore, the highest weight component $\Psi$ carries the charges $S_{1}=1 / 2$ and $S_{2}=-1 / 2$. Therefore, the operators from the $\mathfrak{s u}(1 \mid 1)$ sector have the following charges: $S_{1}=M / 2, S_{2}=-M / 2, J_{1}=M / 2, J_{2}=M / 2$ and $J_{3}=J$.

Coming back to string theory we note that out of eight fermions $\eta$ and $\theta$ only $\theta_{1}$ and $\theta_{2}$ are neutral under the $\mathfrak{s u}(3)$ subgroup, and, therefore, they are dual to the components of the gaugino $\Psi_{\alpha}$. From the table of charges in appendix A we see that $\theta_{1}$ should be identified with the highest weight component $\Psi$. Thus, in the BMN limit the string states dual to operators from the $\mathfrak{s u}(1 \mid 1)$ sector are the states

$$
\left|\Psi_{s u(1 \mid 1)}\right\rangle=\theta_{1, n_{M}}^{+} \theta_{1, n_{M-1}}^{+} \cdots \theta_{1, n_{1}}^{+}|0\rangle .
$$

As was shown in [28], in the uniform light-cone gauge the string theory reduced to the $\mathfrak{s u}(1 \mid 1)$ sector is described by a free fermion. We can also see that from our quartic Hamiltonian (5.24). There is, therefore, no $1 / P_{+}$correction to the free spectrum of the state $(7.13)$, and the 
$1 / J$ correction is just obtained by expanding the frequencies $\omega_{n}$ in powers of $1 / J$. The result of the simple computation is [28]

$$
E_{s u(1 \mid 1)}-J=\sum_{k=1}^{M} \bar{\omega}_{k}-\frac{\lambda^{\prime}}{4 J} \sum_{k=1}^{M} \sum_{j=1}^{M} \frac{n_{k}^{2} \bar{\omega}_{j}^{2}+n_{j}^{2} \bar{\omega}_{k}^{2}}{\bar{\omega}_{k} \bar{\omega}_{j}}
$$

This correction was first computed in [32] by using the uniform gauge $t=\tau, p_{\phi}=J$ in which the $\mathfrak{s u}(1 \mid 1)$ sector is described by a nontrivial integrable model of an interacting Dirac fermion. It was also guessed in [42] by analysing the known 3-impurity result [19].

\section{4. $\mathfrak{s u}(1 \mid 2)$ sector}

The $\mathfrak{s u}(1 \mid 2)$ sector can be considered as the union of the $\mathfrak{s u}(2)$ and $\mathfrak{s u}(1 \mid 1)$ sectors [16], because it consists of operators of the form

$$
O_{s u(1 \mid 2)}=\operatorname{tr}\left(Z^{J-\frac{M}{2}} \Psi^{M} X^{K}+\text { permutations }\right) .
$$

Since we already know that $X$ and $\Psi$ correspond to $\alpha_{1}^{+}$and $\theta_{1}^{+}$, respectively, string theory states dual to the operators (7.15) are of the form

$$
\left|\Psi_{s u(1 \mid 2)}\right\rangle=\left|\Psi_{s u(1 \mid 1)}\right\rangle \otimes\left|\Psi_{s u(2)}\right\rangle=\theta_{1, n_{M}}^{+} \theta_{1, n_{M-1}}^{+} \cdots \theta_{1, n_{1}}^{+} \cdot \alpha_{1, j_{K}}^{+} \alpha_{1, j_{K-1}}^{+} \cdots \alpha_{1, j_{1}}^{+}|0\rangle,
$$

where the mode numbers satisfy the level-matching condition

$$
\sum_{i=1}^{M} n_{i}+\sum_{m=1}^{K} j_{m}=0
$$

This time, however, there is a mixing of states with the same numbers $M$ and $K$, because any state obtained from (7.16) by a permutation of the mode numbers $n_{i}$ and $j_{m}$ has the same energy as the state (7.16) has. If all mode numbers are different the number of all these states is equal to $\frac{(M+K) !}{M ! K !}$, which makes the problem of computing the $1 / P_{+}$correction highly nontrivial. Still the fact that the number of fermions and bosons is the same for all these states appears to make the problem feasible.

\section{5. $\mathfrak{s u}(2 \mid 3)$ sectors}

The $\mathfrak{s u}(2 \mid 3)$ sector [13] is an extension of the $\mathfrak{s u}(1 \mid 2)$ sector. It consists of operators of the form

$$
O_{s u(2 \mid 3)}=\operatorname{tr}\left(Z^{J-\frac{M_{+}}{2}-\frac{M_{-}}{2}} X^{J_{1}} Y^{J_{2}} \Psi_{+}^{M_{+}} \Psi_{-}^{M_{-}}+\text {permutations }\right),
$$

where $\Psi_{+}$is the highest weight component of the gaugino $\Psi_{\alpha}$ from the vector multiplet that was denoted as $\Psi$ in previous subsections, and $\Psi_{-}$is the lowest weight component. String theory states dual to the operators (7.17) are of the form

$$
\left|\Psi_{s u(2 \mid 3)}\right\rangle=\theta_{2, n_{M_{-}}}^{+} \cdots \theta_{2, n_{1}}^{+} \cdot \theta_{1, m_{M_{+}}}^{+} \cdots \theta_{1, m_{1}}^{+} \cdot \alpha_{2, k_{J_{2}}}^{+} \cdots \alpha_{2, k_{1}}^{+} \cdot \alpha_{1, l_{J_{1}}}^{+} \cdots \alpha_{1, l_{1}}^{+}|0\rangle .
$$

In the plane wave limit the space of these string states is highly degenerate. Just as it was for string states from the $\mathfrak{s u}(1 \mid 2)$ sector, we can permute mode numbers of fermions and bosons. Then, a new feature appears in the $\mathfrak{s u}(2 \mid 3)$ sector. One can easily check by using the tables of charges in appendix A that the operators $\alpha_{2, n} \alpha_{1, m}$ and $\theta_{2, k} \theta_{1, l}$ have the same charges $S_{i}$ and $J_{i}$, and, therefore, we can replace any pair of operators $\alpha_{2, n}$ and $\alpha_{1, m}$ in the state (7.18) by a pair of operators $\theta_{2, n}$ and $\theta_{1, m}$ (or $\theta_{2, m}$ and $\theta_{1, n}$ ) with the same mode numbers $n$ and $m$ without changing the plane wave energy of the state. Thus, there is a mixing of states with different numbers of bosons and fermions. This new feature is a string theory analogue of the dynamic 
nature of the long-range spin chain that describes the $\mathfrak{s u}(2 \mid 3)$ sector in $\mathcal{N}=4 \mathrm{SYM}$ [13]. Let us note, however, that in string theory only the states with the same total number of creation operators, $M=J_{1}+J_{2}+M_{-}+M_{+}$, can mix. It seems hardly possible to compute the $1 / P_{+}$ correction to an arbitrary $\mathfrak{s u}(2 \mid 3)$ string state by using conventional methods.

String states dual to all the remaining closed sectors of $\mathcal{N}=4$ SYM can also be easily identified by using the tables of charges from appendix A. Let us also comment that the main obstacle in explicit computations of energy shift for arbitrary $M$-excitation state which originated from the large mixing problem can be significantly reduced if one assumes quantum integrability of the full model (at order $1 / P_{+}$). If the system is integrable, it is enough to determine the energy shift of the arbitrary 3-excitation state. The energy shift for the $M$-excitation state is then given by the sum over the corrected energies of individual impurities (magnons).

\subsection{Effective Hamiltonians for closed sectors}

As was discussed above in this section, the eigenstates of the complete Hamiltonian depend on all the $8+8$ creation operators even for string states dual to operators from closed gauge theory sectors. On the other hand, according to the AdS/CFT correspondence, the string Hamiltonian should be equivalent to the dilatation operator. This means that there should exist a unitary transformation such that the transformed Hamiltonian would have properties similar to those of the dilatation operator; in particular, its action on string states dual to operators from closed sectors would be closed.

We will show here that assuming the finiteness of the quantum theory such a unitary transformation exists in perturbation theory around the plane wave.

In this section, we denote the creation and annihilation operators as $A_{a, n}^{ \pm}$, where $a$ is an index that distinguishes operators of different types, and $n$ is a mode number. The quantum string Hamiltonian will be of the form

$$
H=H_{2}+\frac{1}{P_{+}} H_{4}+\frac{1}{P_{+}^{2}} H_{6}+\cdots .
$$

Here the quadratic Hamiltonian $H_{2}$ reads

$$
H_{2}=\sum_{a, n} \omega_{a, n} A_{a, n}^{+} A_{a, n}^{-}
$$

where the frequencies may in general depend on $a$ and $P_{+}$,

$$
\omega_{a, n}=\omega_{n}+\frac{1}{P_{+}} \omega_{n}^{(1)}+\frac{1}{P_{+}^{2}} \omega_{n}^{(2)}+\cdots .
$$

The quartic Hamiltonian $\mathrm{H}_{4}$ is of the most general form

$$
\begin{gathered}
H_{4}=\sum_{a, n ; b, m ; c, k ; d, l} g_{a, n ; b, m ; c, k ; d, l}^{++++} A_{a, n}^{+} A_{b, m}^{+} A_{c, k}^{+} A_{d, l}^{+}+g_{a, n ; b, m ; c, k ; d, l}^{+++-} A_{a, n}^{+} A_{b, m}^{+} A_{c, k}^{+} A_{d, l}^{-}+\text {h.c. } \\
+g_{a, n ; b, m ; c, k ; d, l}^{++--} A_{a, n}^{+} A_{b, m}^{+} A_{c, k}^{-} A_{d, l}^{-},
\end{gathered}
$$

where the coupling constants $g_{a, n ; b, m ; c, k ; d, l}$ may also depend on $1 / P_{+}$. The remaining Hamiltonians $H_{6}$ and higher are also assumed to be of the most general form. For simplicity, we restrict the consideration to the quartic Hamiltonian.

First of all we show that there exists a unitary transformation that removes all terms with different numbers of creation and annihilation operators. The construction is perturbative in $1 / P_{+}$, and the unitary transformation is of the form

$$
U=\mathrm{e}^{V}, \quad V=\frac{1}{P_{+}} V_{4}+\frac{1}{P_{+}^{2}} V_{6}+\cdots,
$$


where $V_{i}$ are polynomials of the $i$ th order in the creation and annihilation operators

$V_{4}=\sum_{a, n ; b, m ; c, k ; d, l} f_{a, n ; b, m ; c, k ; d, l}^{++++} A_{a, n}^{+} A_{b, m}^{+} A_{c, k}^{+} A_{d, l}^{+}+f_{a, n ; b, m ; c, k ; d, l}^{+++-} A_{a, n}^{+} A_{b, m}^{+} A_{c, k}^{+} A_{d, l}^{-}-$h.c.

Under the unitary transformation the Hamiltonian transforms as follows:

$$
H \rightarrow U H U^{\dagger} .
$$

It is not difficult to see that to remove all unwanted terms from (7.22) at the leading order in $1 / P_{+}$we should make the following choice of the constants $f_{a, n ; b, m ; c, k ; d, l}$ :

$$
f_{a, n ; b, m ; c, k ; d, l}^{++++}=\frac{g_{a, n ; b, m ; c, k ; d, l}^{++++}}{\omega_{a, n}+\omega_{b, m}+\omega_{c, k}+\omega_{d, l}}, \quad f_{a, n ; b, m ; c, k ; d, l}^{+++-}=\frac{g_{a, n ; b, m ; c, k ; d, l}^{+++-}}{\omega_{a, n}+\omega_{b, m}+\omega_{c, k}-\omega_{d, l}} .
$$

It is important to stress that since in perturbation theory $\omega_{a, n}+\omega_{b, m}+\omega_{c, k}-\omega_{d, l}$ is not equal to 0 for any choice of the mode numbers, the unitary transformation is well defined ${ }^{12}$.

Then up to terms of order $1 / P_{+}^{2}$ the Hamiltonian $H_{4}$ takes the form

$$
H_{4}=\sum_{a, n ; b, m ; c, k ; d, l} g_{a, n ; b, m ; c, k ; d, l}^{++--} A_{a, n}^{+} A_{b, m}^{+} A_{c, k}^{-} A_{d, l}^{-} .
$$

The unitary transformation induces additional unwanted terms of order $1 / P_{+}^{2}$ but all these terms can be removed by a similar unitary transformation. The unitary transformations at a higher order in $1 / P_{+}$will typically also induce corrections to the functions $g_{a, n ; b, m ; c, k ; d, l}^{++--}$. It is clear that the procedure can be carried out to any order in $1 / P_{+}$. The resulting Hamiltonian hence contains only terms with an equal number of creation and annihilation operators, with the coefficients which are functions of $1 / P_{+}$. This Hamiltonian can be considered as a string analogue of the field theory dilatation operator because as we will see in a moment it maps a state from a closed sector to another state of this sector ${ }^{13}$.

To simplify the notation, we concentrate our attention on the $\mathfrak{s u}(2)$ sector, but the conclusion is valid for all closed subsectors. Let us assume that we act by the 'diagonal' Hamiltonian of the form (7.25) on a state from the $\mathfrak{s u}(2)$ sector. Then only the terms which do not contain any other annihilation operators but $\alpha_{1, n}^{-}$can produce a nontrivial state. All other terms acting on an $\mathfrak{s u}(2)$ state produce the vacuum. Finally, taking into account that the product of $M$ operators $\alpha_{1, n}^{-}$carries the minimal charge $J_{1}=-M$, we find that the only way to compensate the charge is to multiply them by $M$ creation operators $\alpha_{1, n}^{+}$. Thus, the relevant terms in the Hamiltonian are just obtained by setting all operators but $\alpha_{1, n}^{ \pm}$to zero, and the action of the resulting Hamiltonian is closed on the $\mathfrak{s u}(2)$ string states. This Hamiltonian can be considered as an effective Hamiltonian for the $\mathfrak{s u}(2)$ sector, and its expansion in powers of $\lambda^{\prime}$ should reproduce the Landau-Lifschitz Hamiltonian derived in $[39,47]$.

One could try to simplify the Hamiltonian (7.25) by using a unitary transformation with $V$ of the form

$$
V=\sum_{a, n ; b, m ; c, k ; d, l} \frac{g_{a, n ; b, m ; c, k ; d, l}^{++--}}{\omega_{a, n}+\omega_{b, m}-\omega_{c, k}-\omega_{d, l}} A_{a, n}^{+} A_{b, m}^{+} A_{c, k}^{-} A_{d, l}^{-} .
$$

If the denominator $\omega_{a, n}+\omega_{b, m}-\omega_{c, k}-\omega_{d, l}$ would never vanish then we could remove all quartic terms. It is clear, however, that in perturbation theory in $1 / P_{+}$it vanishes if $k=n, l=m$

\footnotetext{
${ }^{12}$ Strictly speaking, since we are dealing with a system with an infinite number of degrees of freedom, to have a well-defined transformation one should introduce an ultraviolet cut-off, for example by replacing $\omega_{a, n}$ by $\omega_{a, n} \mathrm{e}^{\epsilon|n|}$ where $\epsilon$ is the regularization parameter. In fact, one would need a regularization parameter even to the normal-order Hamiltonian.

${ }^{13}$ Note that this is not necessarily in contradiction with Minahan [46], who argued for the non-perturbative violation of the closedness of the $\mathfrak{s u}(2)$ sector, as our argument here is purely perturbative.
} 
or $k=m, l=n$, and therefore these terms cannot be removed. One can show that the denominator does not vanish for any other choice of mode numbers, and, therefore, we can reduce (7.25) to the following simple form:

$$
H_{4}=\sum_{a, n ; b, m ; c ; d} g_{a, n ; b, m ; c ; d}^{++--} A_{a, n}^{+} A_{b, m}^{+} A_{c, n}^{-} A_{d, m}^{-} .
$$

A Hamiltonian of this form allows a straightforward computation of the energy of string states from rank-1 closed sectors.

\section{Quantum string light-cone Bethe equations}

It has been proposed in [24] that the energies $E$ of the $\mathrm{AdS}_{5} \times \mathrm{S}^{5}$ quantum string, as measured with respect to the global time coordinate $t$, should arise as solutions of a set of quantum string Bethe equations. The energy $E$ is natural from the perspective of comparing with the dual gauge theory and its scaling dimensions. However, as we have seen in the previous sections, the energy in global time $E$ is not the most natural quantity for the purposes of the quantization of the $\mathrm{AdS}_{5} \times \mathrm{S}^{5}$ string in light-cone gauge, but rather the world-sheet Hamiltonian $H_{\mathrm{lc}}=E-J$. A natural question to ask now is, whether it is possible to write down a set of light-cone quantum string Bethe equations directly yielding the spectrum of $H_{\mathrm{lc}}$. The expectation is that this set of equations takes a simpler form than the quantum string Bethe equations of $[16,24,43]$ directly leading to $E$.

For this let us now assume that the quantum string is integrable in the sense that the elementary world-sheet excitations ('magnons') interact with each other only via two-body interactions, described by the $S$-matrix, as was advocated by Staudacher [43], whose logic we closely follow. The system should then be described by the fundamental equation

$$
\frac{P_{+}}{2} p_{k}=2 \pi n_{k}+\sum_{j \neq k}^{M} \theta\left(p_{k}, p_{j}\right)
$$

where the $S$-matrix is given by $S(p, q)=\exp [\mathrm{i} \theta(p, q)]$. In the plane wave limit, at the leading order in the $1 / P_{+}$expansion, the system is free (i.e. $\theta\left(p_{j}, p_{k}\right)=0$ ). Thus $p_{k}$ has the perturbative expansion for $P_{+} \rightarrow \infty$ with $\tilde{\lambda}$ of (5.14) held fixed,

$$
p_{k}=\frac{4 \pi}{P_{+}} n_{k}+\frac{\delta p_{k}}{P_{+}^{2}}+\mathcal{O}\left(\frac{1}{P_{+}^{3}}\right)
$$

where $\delta p_{k}$ are corrections. From this one determines that

$$
\delta p_{k}=2 P_{+} \sum_{j \neq k}^{M} \theta\left(\frac{4 \pi n_{k}}{P_{+}}, \frac{4 \pi n_{j}}{P_{+}}\right) .
$$

Next, we take our elementary excitations to satisfy the dispersion relation

$$
E_{\mathrm{lc}}\left(p_{k}\right)=\sqrt{1+\frac{\lambda}{4 \pi^{2}} p_{k}^{2}},
$$

and take the total world-sheet energy $H_{\mathrm{lc}}$ to be additive $E_{\mathrm{lc}}=\sum_{k=1}^{M} E_{\mathrm{lc}}\left(p_{k}\right)$. Inserting the corrections $\delta p_{k}$ of (8.3) and (8.2) into the dispersion relation (8.9), we deduce an expression for the shift of $P_{-}$at order $1 / P_{+}^{2}$ in terms of the $S$-matrix,

$$
\delta P_{-}=\frac{\tilde{\lambda} P_{+}}{2 \pi} \sum_{k, j=1, k \neq j}^{M} \frac{n_{k}}{\sqrt{1+\tilde{\lambda} n_{k}^{2}}} \theta\left(\frac{4 \pi}{P_{+}} n_{k}, \frac{4 \pi}{P_{+}} n_{j}\right) .
$$


On the other hand, the semiclassical quantization of the string yielded for the $E_{\mathrm{lc}}$ shift equations (7.5) and (7.9). By comparing (8.5) to (7.5) and (7.9), we can extract the $S$-matrix at leading order $1 / P_{+}^{2}$,

$$
\begin{aligned}
& \theta\left(p_{k}, p_{j}\right)=-\frac{\mathfrak{s}}{2} \frac{\left(p_{k}+p_{j}\right)^{2}}{p_{k} \omega_{j}-p_{j} \omega_{k}} \quad \text { where } \begin{cases}\mathfrak{s}=1 & \text { for } \mathfrak{s u}(2) \\
\mathfrak{s}=0 & \text { for } \mathfrak{s u}(1 \mid 1) \\
\mathfrak{s}=-1 & \text { for } \mathfrak{s l}(2)\end{cases} \\
& \omega_{k}=\sqrt{1+\frac{\lambda}{4 \pi^{2}} p_{k}^{2}} .
\end{aligned}
$$

This near plane wave $S$-matrix is singular at $p_{k}=p_{j}$. It is clear, however, that this singularity is an artefact of the expansion in $1 / P_{+}$similar to the singularity of the $\mathrm{XXX}$ spin chain $S$-matrix in the long spin chain length limit. It turns out that the fundamental relation (8.1) with the above expression for the scattering phase shift $\theta\left(p_{k}, p_{j}\right)$ follows from the very compact set of light-cone Bethe equations with an $S$-matrix regular at $p_{k}=p_{j}$,

$$
\exp \left(\mathrm{i} \frac{P_{+}}{2} p_{k}\right)=\prod_{j=1, j \neq k}^{M}\left(\frac{x_{k}^{+}-x_{j}^{-}}{x_{k}^{-}-x_{j}^{+}} \mathrm{e}^{\mathrm{i}\left(p_{j}-p_{k}\right)}\right)^{\mathfrak{s}},
$$

where we have used the common variables $x_{k}^{ \pm}=x^{ \pm}\left(p_{k}\right)$ with

$$
x^{ \pm}(p)=\frac{1}{4}\left(\cot \frac{p}{2} \pm \mathrm{i}\right)\left(1+\sqrt{1+\frac{\lambda}{\pi^{2}} \sin ^{2} \frac{p}{2}}\right),
$$

first introduced in [30]. It can be checked that this $S$-matrix, after rescaling $p_{k} \rightarrow 2 p_{k} / P_{+}$and expanding in $1 / P_{+}$, indeed reduces to the scattering phase of equations (8.6). Moreover, it is then natural to assume a generalized dispersion relation of the form

$$
E_{\mathrm{lc}}^{\mathrm{gen}}\left(p_{k}\right)=\sqrt{1+\frac{\lambda}{\pi^{2}} \sin ^{2}\left(\frac{p_{k}}{2}\right)}
$$

however the potential sine structure would only manifest itself in the next-to-leading corrections to the plane wave limit of order $1 / P_{+}$.

In addition, we are only considering translationally invariant states as a consequence of the level-matching condition such that

$$
\sum_{j=1}^{M} p_{j}=0
$$

Using this our light-cone Bethe equations can be rewritten in an even simpler form as

$$
\exp \left(\mathrm{i} \frac{P_{+}+\mathfrak{s} M}{2} p_{k}\right)=\prod_{j=1, j \neq k}^{M}\left(\frac{x_{k}^{+}-x_{j}^{-}}{x_{k}^{-}-x_{j}^{+}}\right)^{\mathfrak{s}} .
$$

The associated dispersion relation for the world-sheet energy $E_{\mathrm{lc}}$ is stated in (8.9).

The light-cone Bethe equations can be used to compute subleading $1 / P_{+}^{2}$ corrections. We do not expect, however, that they will produce the correct result. In particular, the anomaly computation of [25] observed a discrepancy between the quantum string Bethe predictions and the results of the semiclassical string quantization. The anomaly in [25] arose purely from the short distance behaviour of the term on the RHS of (8.11), while the other terms which are different in (8.11) with respect to equations of ([24]) did not contribute. Thus, the anomaly prediction from (8.11) will be the same as those in [25], and hence will not cure the problem which ([24]) faced. 
The Bethe equations (8.7) have been derived by using the expansion around the plane wave. On the other hand, one should expect them to also reproduce the leading $\lambda^{1 / 4}$ asymptotic behaviour of short strings in the strong coupling (flat space) limit and the finite-gap integral equations of [44, 45] which describe strings spinning in $R \times S^{3}$ and $\operatorname{AdS}_{3} \times R$ in the scaling limit of [21], just as the quantum string Bethe equations of [24] did.

\subsection{Strong coupling limit}

The leading $\lambda^{1 / 4}$ asymptotic behaviour of short strings in the strong coupling (flat space) limit was discussed in detail for the $\mathfrak{s u}(1 \mid 1)$ sector in [28]. The consideration there is also valid for the $\mathfrak{s u}(2)$ and $\mathfrak{s l}(2)$ sectors because as one can easily see the $S$-matrix does not contribute in the strong coupling limit $\lambda \rightarrow \infty, \lambda / P_{+}^{4}$ fixed: the quasi-momenta then have an expansion of the form

$$
p_{k}^{\text {s.c. }}=\frac{p_{k}^{0}}{\lambda^{1 / 4}}+\frac{p_{k}^{1}}{\lambda^{1 / 2}}+\cdots
$$

Then obviously the scattering phase of (8.6) scales as $\theta\left(p_{k}, p_{j}\right) \sim \frac{1}{\sqrt{\lambda}}$ in consequence of $\omega\left(p_{k}\right) \sim \lambda^{1 / 4}$. Therefore the fundamental equation (8.1) yields

$p_{k}^{0}=\frac{4 \pi n_{k}}{E^{(0)}} \quad$ where $\quad P_{+}=E+J=\lambda^{1 / 4} E^{(0)}+E^{(1)}+J+\mathcal{O}\left(\lambda^{-1 / 4}\right)$.

Plugging this into the dispersion relation (8.4) we find

$$
E_{\mathrm{lc}}=\lambda^{1 / 4} E^{(0)}+\mathcal{O}(1)=\sum_{k=1}^{M} \lambda^{1 / 4} \frac{2\left|n_{k}\right|}{E^{(0)}}+\mathcal{O}(1) .
$$

Due to the level-matching condition $\sum_{k} n_{k}=0$ and one may define the level number $n$ as the sum over the positive $n_{k}$. This implies $\sum_{k}\left|n_{k}\right|=2 n$. With this definition, we indeed reproduce the result of [7] upon solving the quadratic equation (8.14) for $E^{(0)}$

$$
E=2 \lambda^{1 / 4} \sqrt{n}+\mathcal{O}(1)
$$

Hence in the light-cone gauge the leading $\lambda^{1 / 4}$ asymptotics just comes from the spectrum of the free harmonic oscillator.

\subsection{Spinning string limit}

To derive the integral equations in the spinning string limit it is convenient to use the logarithmic form (8.1) of the Bethe equations (8.7). To take the limit we rescale momenta as $p_{k} \rightarrow 2 p_{k} / P_{+}$ and introduce the distributional density [48]

$$
\rho(p)=\frac{2}{P_{+}} \sum_{k=1}^{M} \delta\left(p-p_{k}\right), \quad \int_{\mathbf{C}} \mathrm{d} p \rho(p)=\frac{2 M}{P_{+}} .
$$

Then in the limit $P_{+} \rightarrow \infty, M / P_{+}$fixed, we get the following integral equation,

$$
p=f_{\mathbf{C}} \mathrm{d} q \rho(q) \theta(p, q)
$$

where

$$
\theta(p, q)=-\frac{\mathfrak{s}}{2} \frac{(p+q)(p \omega(q)+q \omega(p))}{p-q}, \quad \omega(p)=\sqrt{1+\frac{\tilde{\lambda}}{4 \pi^{2}} p^{2}}
$$


This equation should be supplemented by the zero-momentum condition

$$
\int_{\mathbf{C}} \mathrm{d} p \rho(p) p=0 .
$$

Then, solving equations (8.16), (8.17) and (8.19), we can find the light-cone energy of a spinning string by using the equation

$$
\frac{2 E_{\mathrm{lc}}}{P_{+}}=\int_{\mathbf{C}} \mathrm{d} p \rho(p) \omega(p) .
$$

To compare equations (8.16), (8.17), (8.19) and (8.20) with the finite-gap integral equations of $[44,45]$ we should start with their unscaled form ${ }^{14}$

$$
\begin{aligned}
& 2 f_{\mathbf{C}} \mathrm{d} y \frac{\rho_{s}(y)}{x-y}=\frac{2 \pi\left(P_{+}+P_{-}\right)}{\sqrt{\lambda}} \frac{x}{x^{2}-\frac{\tilde{\lambda}}{16 \pi^{2}}}, \\
& \int_{\mathbf{C}} \mathrm{d} x \frac{\rho_{s}(x)}{x}=2 \pi m=0, \\
& \int_{\mathbf{C}} \mathrm{d} x \rho_{s}(x)=\frac{2 \pi}{\sqrt{\lambda}}\left(P_{-}+M\right), \\
& \int_{\mathbf{C}} \mathrm{d} x \frac{\rho_{s}(x)}{x^{2}}=\frac{2 \pi}{\sqrt{\lambda}}\left(P_{-}-M\right),
\end{aligned}
$$

where we set the winding number to 0 because our Bethe equations have been derived under this assumption.

First, we rescale the spectral parameter as $x \rightarrow 2 \pi P_{+} x / \sqrt{\lambda}$. This rescaling should be contrasted to the rescaling $x \rightarrow 4 \pi(J+M) x / \sqrt{\lambda}$ performed in [44], in order to achieve the comparison with the gauge theory Bethe ansatz [16].

Then, it is easy to see that after the rescaling, the set of equations $(8.21),(8.22),(8.23)$ and (8.24) can be written in the form

$$
\begin{aligned}
& \int_{\mathbf{C}} \mathrm{d} x \frac{\rho_{s}(x)}{x}=0 \\
& \int_{\mathbf{C}} \mathrm{d} x \rho_{s}(x)\left(1-\frac{\tilde{\lambda}}{16 \pi^{2}} \frac{1}{x^{2}}\right)=\frac{2 M}{P_{+}} \\
& \frac{2 P_{-}}{P_{+}}=\int_{\mathbf{C}} \mathrm{d} x \rho_{s}(x)\left(1+\frac{\tilde{\lambda}}{16 \pi^{2}} \frac{1}{x^{2}}\right) \\
& \frac{x}{x^{2}-1}=2 f_{\mathbf{C}} \mathrm{d} y \frac{\rho_{s}(y)}{x-y}-\frac{P_{-}}{P_{+}} \frac{x}{x^{2}-1} .
\end{aligned}
$$

Finally, making the change of the spectral parameter $x$

$$
p=\frac{x}{x^{2}-\frac{\tilde{\lambda}}{16 \pi^{2}}}, \quad \rho(p)=\frac{\rho_{s}(x)}{p^{2} \omega(p)},
$$

we find that the finite-gap integral equations (8.28), (8.25), (8.27) and (8.26) coincide with the equations (8.16), (8.17), (8.19) and (8.20) we derived in the spinning string limit.

${ }^{14}$ We restrict our attention to the $\mathfrak{s u}(2)$ sector because the consideration of the equations in the $\mathfrak{s l}(2)$ sector literally repeats the one we do for the $\mathfrak{s u}(2)$ sector. 


\section{Conclusions and outlook}

The bulk of this paper consisted in a rather laborious derivation of the exact uniform light-cone and $\kappa$-symmetry gauge-fixed Lagrangian in a first-order formalism. This enables one to read off the (involved) Poisson structure and form of the light-cone Hamiltonian $H_{\mathrm{lc}}=-p_{-}$of the $\mathrm{AdS}_{5} \times \mathrm{S}^{5}$ superstring. We then went on to quantize this system in the near plane wave limit of taking the constant light-cone momentum $P_{+}$to infinity while keeping $\tilde{\lambda}=4 \lambda / P_{+}^{2}$ fixed. In this limit, we could systematically expand the Hamiltonian to the quartic order in physical fields and study the leading energy shifts of the closed rank-1 subsectors for general states, reproducing the results of Callan et al [19] in a rather economical fashion. Furthermore, we proved the existence of effective Hamiltonians in closed subsectors of the theory, which are the analogues of the dilatation operators in the closed subsectors of the gauge theory. Finally we were able to write down a novel, compact set of light-cone Bethe equations, which captures the leading $1 / P_{+}$energy shifts in the rank-1 subsectors of the quantum superstring. This set of quantum string equations was shown to possess the correct strong coupling and spinning string limits known in the literature.

There are numerous extensions of the present work. First of all the next-order energy shift computation for $H_{\mathrm{lc}}$ in the $1 / P_{+}$expansion is now within reach and should be performed. It will be able to test the sine structure in the dispersion relation for the energy. Here subtle quantum ordering ambiguities have to be overcome: at the leading $1 / P_{+}$order our simple normal ordering prescription was justified by the requirement of having an unmodified quadratic Hamiltonian. At the next order such a simple normal ordering prescription fails, as it would lead to energy shifts of protected states. We believe that imposing the closure of the algebra of the system will pave us the correct path to resolve these ordering ambiguities at the quantum level. We are presently investigating this issue.

The uniform light-cone gauge Hamiltonian we have established should be the basis of an investigation of the near flat space limit of $\mathrm{AdS}_{5} \times \mathrm{S}^{5}$. Here the subtlety lies in the treatment of the zero modes, which in a naive approach leads to a breakdown of the perturbation theory about the flat space point.

Another obvious question is how to generalize our findings to the case of non-vanishing winding numbers. This should be relevant for the study of $\gamma$-deformed models [35, 36] which are known to be related to Green-Schwarz superstrings in $\mathrm{AdS}_{5} \times \mathrm{S}^{5}$ subject to twisted boundary conditions $[34,36]$. The twisting effectively corresponds to a non-vanishing (and non-integer) mode number in the expansion around plane wave limits. In the $\gamma$-deformed case there are (at least) two inequivalent plane wave limits [35-37] and the light-cone-type gauges seem to be very convenient to study $1 / J$ corrections to energies of string states in these limits.

One might also try to find explicit string solutions of our exact gauge-fixed Lagrangian and study quantum fluctuations about these. In particular finding circular string would be interesting. It would allow for a computation of the $1 / J^{2}$ corrections to the energies of such circular strings that could be potentially simpler than the computations in the static gauge [21] used before.

Our newly proposed quantum string light-cone Bethe equations call for a number of checks and extensions. Their generalization to the full $P S U(2,2 \mid 4)$ structure seems obvious to guess in view of [16]. This could be checked by a parallel independent computation of the energy shifts in larger subsectors based on our quartic Hamiltonian. Finally, we have seen that focusing on the computation of the corrections to light-cone energy $\Delta-J$ instead of the dilatation operator $\Delta$ has lead to the simplified Bethe equations on the string side: the dressing factor has 'disappeared'. It would be very interesting to understand what are the 
corresponding changes for the (asymptotic) Bethe equations in the dual gauge theory, once they are rephrased in the language of the light-cone variables.

We intend to return to some of these questions in future works.

\section{Acknowledgments}

We wish to thank Gleb Arutyunov, Niklas Beisert, Matthias Staudacher and Arkady Tseytlin for very useful discussions and comments. The work of JP is funded by the Volkswagen Foundation. He also thanks the Max-Planck-Institute for Gravitational Physics for hospitality. The work of SF and MZ was supported in part by the EU-RTN network Constituents, Fundamental Forces and Symmetries of the Universe (MRTN-CT-2004-005104).

\section{Appendix A. Dirac matrices}

Throughout the paper we will use the following explicit representation of Dirac matrices,

$$
\begin{array}{rlrl}
\gamma^{1} & =\left(\begin{array}{cccc}
0 & 0 & 0 & -1 \\
0 & 0 & 1 & 0 \\
0 & 1 & 0 & 0 \\
-1 & 0 & 0 & 0
\end{array}\right), & \gamma^{2}=\left(\begin{array}{cccc}
0 & 0 & 0 & \mathrm{i} \\
0 & 0 & \mathrm{i} & 0 \\
0 & -\mathrm{i} & 0 & 0 \\
-\mathrm{i} & 0 & 0 & 0
\end{array}\right), \quad \gamma^{3}=\left(\begin{array}{cccc}
0 & 0 & 1 & 0 \\
0 & 0 & 0 & 1 \\
1 & 0 & 0 & 0 \\
0 & 1 & 0 & 0
\end{array}\right), \\
\gamma^{4}=\left(\begin{array}{cccc}
0 & 0 & -\mathrm{i} & 0 \\
0 & 0 & 0 & \mathrm{i} \\
\mathrm{i} & 0 & 0 & 0 \\
0 & -\mathrm{i} & 0 & 0
\end{array}\right), & \gamma^{5}=\left(\begin{array}{cccc}
1 & 0 & 0 & 0 \\
0 & 1 & 0 & 0 \\
0 & 0 & -1 & 0 \\
0 & 0 & 0 & -1
\end{array}\right)=\Sigma,
\end{array}
$$

satisfying the $S O(5)$ Clifford algebra

$$
\gamma^{a} \gamma^{b}+\gamma^{b} \gamma^{a}=2 \delta_{a b}
$$

Moreover, all of them are Hermitian, so that $\mathrm{i} \gamma_{a}$ belongs to $\mathfrak{s u}(4)$.

It is also useful to introduce such a basis that all bosonic fields have definite charges under the $U(1)$ subgroups of $\operatorname{PSU}(2,2 \mid 4)$. We also describe a convenient parametrization of the fermionic matrices $\chi$ and $\Theta$.

The complex fields carrying definite charges are

$$
\begin{array}{llll}
Z_{1}=z_{2}+\mathrm{i} z_{1}, & Z_{2}=z_{4}+\mathrm{i} z_{3}, & Z_{\overline{1}}=z_{2}-\mathrm{i} z_{1}, & Z_{\overline{2}}=z_{4}-\mathrm{i} z_{3}, \\
Y_{1}=y_{2}+\mathrm{i} y_{1}, & Y_{2}=y_{4}+\mathrm{i} y_{3}, & Y_{\overline{1}}=y_{2}-\mathrm{i} y_{1}, & Y_{\overline{2}}=y_{4}-\mathrm{i} y_{3} .
\end{array}
$$

We want to have the identity

$$
z_{i} \gamma_{i}=Z_{a} \Gamma_{a}=Z_{1} \Gamma_{1}+Z_{\overline{1}} \Gamma_{\overline{1}}+Z_{2} \Gamma_{2}+Z_{\overline{2}} \Gamma_{\overline{2}},
$$

which lets us introduce

$$
\begin{aligned}
& \Gamma_{1}=\frac{1}{2}\left(\gamma_{2}-\mathrm{i} \gamma_{1}\right)=\left(\begin{array}{cccc}
0 & 0 & 0 & \mathrm{i} \\
0 & 0 & 0 & 0 \\
0 & -\mathrm{i} & 0 & 0 \\
0 & 0 & 0 & 0
\end{array}\right), \quad \Gamma_{\overline{1}}=\Gamma_{1}^{\dagger}=\frac{1}{2}\left(\gamma_{2}+\mathrm{i} \gamma_{1}\right)=\left(\begin{array}{cccc}
0 & 0 & 0 & 0 \\
0 & 0 & \mathrm{i} & 0 \\
0 & 0 & 0 & 0 \\
-\mathrm{i} & 0 & 0 & 0
\end{array}\right) \\
& \Gamma_{2}=\frac{1}{2}\left(\gamma_{4}-\mathrm{i} \gamma_{3}\right)=\left(\begin{array}{cccc}
0 & 0 & -\mathrm{i} & 0 \\
0 & 0 & 0 & 0 \\
0 & 0 & 0 & 0 \\
0 & -\mathrm{i} & 0 & 0
\end{array}\right), \quad \Gamma_{\overline{2}}=\Gamma_{2}^{\dagger}=\frac{1}{2}\left(\gamma_{4}+\mathrm{i} \gamma_{3}\right)=\left(\begin{array}{cccc}
0 & 0 & 0 & 0 \\
0 & 0 & 0 & \mathrm{i} \\
\mathrm{i} & 0 & 0 & 0 \\
0 & 0 & 0 & 0
\end{array}\right) \text {. }
\end{aligned}
$$


In what follows, we will often use for the indices $\overline{1}$ and $\overline{2}$ the following convention:

$$
\overline{1} \equiv 4, \quad \overline{2} \equiv 3, \quad \Rightarrow \quad \bar{a} \equiv 5-a, \quad a=1,2,3,4 .
$$

In particular, this means that

$$
\Gamma_{\overline{1}} \equiv \Gamma_{4}, \quad \Gamma_{\overline{2}} \equiv \Gamma_{3}, \quad \Gamma_{\bar{a}} \equiv \Gamma_{5-a}, \quad \Gamma_{a}^{\dagger} \equiv \Gamma_{5-a} .
$$

It is also useful to introduce the following two orthogonal projectors:

$$
\mathcal{P}_{+}=\frac{1}{2}\left(I_{4}+\Sigma\right)=\left(\begin{array}{cc}
I_{2} & 0 \\
0 & 0
\end{array}\right), \quad \mathcal{P}_{-}=\frac{1}{2}\left(I_{4}-\Sigma\right)=\left(\begin{array}{cc}
0 & 0 \\
0 & I_{2}
\end{array}\right) .
$$

We can write the fermionic $8 \times 8$ matrix $\chi$ in the form

$$
\chi=\left(\begin{array}{cc}
0 & \Theta \\
\Theta_{*} & 0
\end{array}\right)=\sigma_{+} \otimes \Theta+\sigma_{-} \otimes \Theta_{*}
$$

where

$$
\sigma_{+}=\left(\begin{array}{ll}
0 & 1 \\
0 & 0
\end{array}\right), \quad \sigma_{-}=\left(\begin{array}{ll}
0 & 0 \\
1 & 0
\end{array}\right)
$$

are the two nilpotent 2 by 2 matrices. Note that the two-dimensional projectors $P_{2}^{ \pm}$are expressed through $\sigma^{ \pm}$as follows:

$$
P_{2}^{+}=\sigma_{+} \sigma_{-}=\left(\begin{array}{ll}
1 & 0 \\
0 & 0
\end{array}\right), \quad P_{2}^{-}=\sigma_{-} \sigma_{+}=\left(\begin{array}{ll}
0 & 0 \\
0 & 1
\end{array}\right) .
$$

Then, the $\kappa$-fixed $\Theta$ can be expanded as follows:

$$
\begin{aligned}
& \Theta=\mathcal{P}_{+} \eta+\mathcal{P}_{-} \theta^{\dagger}, \quad \eta=\eta_{a} \Gamma_{a}, \quad \theta=\theta_{a} \Gamma_{a} \\
& \Theta^{\dagger}=\eta^{\dagger} \mathcal{P}_{+}+\theta \mathcal{P}_{-}=\mathcal{P}_{-} \eta^{\dagger}+\mathcal{P}_{+} \theta \\
& \Theta_{*}=-\Theta^{\dagger} \Sigma=-\eta^{\dagger} \mathcal{P}_{+}+\theta \mathcal{P}_{-}=-\mathcal{P}_{-} \eta^{\dagger}+\mathcal{P}_{+} \theta
\end{aligned}
$$

The fermions $\theta_{i j}$ are related to $\eta_{a}$ and $\theta_{a}$ as follows:

$$
\begin{array}{llll}
\theta_{13}=-\mathrm{i} \eta_{2}, & \theta_{14}=\mathrm{i} \eta_{1}, & \theta_{23}=\mathrm{i} \eta_{4}, & \theta_{24}=\mathrm{i} \eta_{3}, \\
\theta_{31}=\mathrm{i} \theta_{2}^{\dagger}, & \theta_{32}=-\mathrm{i} \theta_{4}^{\dagger}, & \theta_{41}=-\mathrm{i} \theta_{1}^{\dagger}, & \theta_{42}=-\mathrm{i} \theta_{3}^{\dagger} .
\end{array}
$$

Since $\Sigma$ anticommutes with all $\Gamma_{a}$ it also anticommutes with the $\kappa$-gauge-fixed $\Theta$ and $\Theta_{*}$. By using this property one can easily show that

$$
\Sigma_{+} \chi=-\chi \Sigma_{+}, \quad \Sigma_{-} \chi=\chi \Sigma_{-} .
$$

The bosonic fields and fermions are charged under the four $U(1)$ subgroups of $S U(2,2) \times S U(4)$ generated by

$$
\Phi_{i}^{\mathrm{AdS}}=P_{2}^{+} \otimes \Phi_{i}, \quad \Phi_{i}^{S}=P_{2}^{-} \otimes \Phi_{i}
$$

where

$$
\Phi_{1}=\frac{1}{2}\left(\begin{array}{cccc}
1 & 0 & 0 & 0 \\
0 & -1 & 0 & 0 \\
0 & 0 & 1 & 0 \\
0 & 0 & 0 & -1
\end{array}\right), \quad \Phi_{2}=\frac{1}{2}\left(\begin{array}{cccc}
1 & 0 & 0 & 0 \\
0 & -1 & 0 & 0 \\
0 & 0 & -1 & 0 \\
0 & 0 & 0 & 1
\end{array}\right)
$$

It is not difficult to check (see [32] for details) that $\Phi_{1}^{\text {AdS }}$ and $\Phi_{2}^{\text {AdS }}$ generate rotations in the planes $z_{2} z_{1}$ and $z_{4} z_{3}$, or multiplication by a phase of $Z_{1}$ and $Z_{2}$, respectively. We denote these $\mathrm{AdS}_{5}$ charges as $S_{i}$. Similarly, $\Phi_{1}^{S}$ and $\Phi_{2}^{S}$ generate rotations in the planes $y_{2} y_{1}$ and $y_{4} y_{3}$, or multiplication by a phase of $Y_{1}$ and $Y_{2}$, respectively. We denote these $\mathrm{S}^{5}$ charges as $J_{i}$. 
In the tables below we list the field charges under the four $U(1)$ subgroups. Let us stress again that all the fields are neutral under the two $U(1)$ subgroups that correspond to shifts of the global time coordinate, and the $S^{5}$ angle $\phi$.

Charges of bosonic fields and creation and annihilation operators:

\begin{tabular}{|l|c|c|c|c|}
\hline & $S_{1}$ & $S_{2}$ & $J_{1}$ & $J_{2}$ \\
\hline$Z_{1}, P_{1}^{z}, \beta_{1, n}^{+}, \beta_{4, n}^{-}$ & +1 & 0 & 0 & 0 \\
\hline$Z_{2}, P_{2}^{z}, \beta_{2, n}^{+}, \beta_{3, n}^{-}$ & 0 & +1 & 0 & 0 \\
\hline$Z_{3}, P_{3}^{z}, \beta_{3, n}^{+}, \beta_{2, n}^{-}$ & 0 & -1 & 0 & 0 \\
\hline$Z_{4}, P_{4}^{z}, \beta_{4, n}^{+}, \beta_{1, n}^{-}$ & -1 & 0 & 0 & 0 \\
\hline
\end{tabular}

\begin{tabular}{|l|c|c|c|c|}
\hline & $S_{1}$ & $S_{2}$ & $J_{1}$ & $J_{2}$ \\
\hline$Y_{1}, P_{1}^{y}, \alpha_{1, n}^{+}, \alpha_{4, n}^{-}$ & 0 & 0 & +1 & 0 \\
\hline$Y_{2}, P_{2}^{y}, \alpha_{2, n}^{+}, \alpha_{3, n}^{-}$ & 0 & 0 & 0 & +1 \\
\hline$Y_{3}, P_{3}^{y}, \alpha_{3, n}^{+}, \alpha_{2, n}^{-}$ & 0 & 0 & 0 & -1 \\
\hline$Y_{4}, P_{4}^{y}, \alpha_{4, n}^{+}, \alpha_{1, n}^{-}$ & 0 & 0 & -1 & 0 \\
\hline
\end{tabular}

Charges of fermions and creation and annihilation operators:

\begin{tabular}{|l|r|r|r|r|}
\hline & $S_{1}$ & $S_{2}$ & $J_{1}$ & $J_{2}$ \\
\hline$\theta_{1}, \theta_{4}^{\dagger}, \theta_{1, n}^{+}, \theta_{4, n}^{-}$ & $+\frac{1}{2}$ & $-\frac{1}{2}$ & $+\frac{1}{2}$ & $+\frac{1}{2}$ \\
\hline$\theta_{2}, \theta_{3}^{\dagger}, \theta_{2, n}^{+}, \theta_{3, n}^{-}$ & $-\frac{1}{2}$ & $+\frac{1}{2}$ & $+\frac{1}{2}$ & $+\frac{1}{2}$ \\
\hline$\theta_{3}, \theta_{2}^{\dagger}, \theta_{3, n}^{+}, \theta_{2, n}^{-}$ & $+\frac{1}{2}$ & $-\frac{1}{2}$ & $-\frac{1}{2}$ & $-\frac{1}{2}$ \\
\hline$\theta_{4}, \theta_{1}^{\dagger}, \theta_{4, n}^{+}, \theta_{1, n}^{-}$ & $-\frac{1}{2}$ & $+\frac{1}{2}$ & $-\frac{1}{2}$ & $-\frac{1}{2}$ \\
\hline
\end{tabular}

\begin{tabular}{|l|r|r|c|c|}
\hline & $S_{1}$ & $S_{2}$ & $J_{1}$ & $J_{2}$ \\
\hline$\eta_{1}, \eta_{4}^{\dagger}, \eta_{1, n}^{+}, \eta_{4, n}^{-}$ & $+\frac{1}{2}$ & $+\frac{1}{2}$ & $+\frac{1}{2}$ & $-\frac{1}{2}$ \\
\hline$\eta_{2}, \eta_{3}^{\dagger}, \eta_{2, n}^{+}, \eta_{3, n}^{-}$ & $+\frac{1}{2}$ & $+\frac{1}{2}$ & $-\frac{1}{2}$ & $+\frac{1}{2}$ \\
\hline$\eta_{3}, \eta_{2}^{\dagger}, \eta_{3, n}^{+}, \eta_{2, n}^{-}$ & $-\frac{1}{2}$ & $-\frac{1}{2}$ & $+\frac{1}{2}$ & $-\frac{1}{2}$ \\
\hline$\eta_{4}, \eta_{1}^{\dagger}, \eta_{4, n}^{+}, \eta_{1, n}^{-}$ & $-\frac{1}{2}$ & $-\frac{1}{2}$ & $-\frac{1}{2}$ & $+\frac{1}{2}$ \\
\hline
\end{tabular}

Below we collect some useful identities:

$$
\begin{array}{ll}
K_{4,8}^{2}=-I_{4,8} & \Sigma_{ \pm}^{2}=I \\
K_{4} \Sigma K_{4}=-\Sigma, & K_{8} \Sigma_{ \pm} K_{8}=-\Sigma_{ \pm} \\
K_{4} \gamma^{i} K_{4}=-\left(\gamma^{i}\right)^{t} & K g^{t} K=-g, \\
K\left(g^{-1}\right)^{t} K=-g^{-1}, & K \partial_{\alpha} g^{t} K=-\partial_{\alpha} g \\
g \Sigma_{ \pm} g^{-1}=g^{2} \Sigma_{ \pm}=\Sigma_{ \pm} g^{-2} . &
\end{array}
$$

\section{Appendix B. Computing the gauge-fixed Lagrangian}

In this appendix, we simplify various terms appearing in the Lagrangian (3.12), solve the Virasoro constraints and find $x_{-}^{\prime}$ and $\pi_{-}$.

\section{B.1. Simplifying $\mathbf{p}_{-}$}

Taking into account the decomposition (3.4) of $\pi$, we rewrite $\mathbf{p}_{-}$(3.15) in the form

$$
\begin{gathered}
\mathbf{p}_{-}=\frac{\pi_{-}}{8} \operatorname{Str}\left(\Sigma_{8}\left(1+2 \chi^{2}\right) g(x)^{2}\right)-\frac{\pi_{+}}{8} \operatorname{Str}\left(\left(1+2 \chi^{2}\right) g(x)^{2}\right) \\
+\frac{\mathrm{i} \pi_{M}}{4} \operatorname{Str}\left(\Sigma_{M} \Sigma_{+} g(x)\left(1+2 \chi^{2}\right) g(x)\right)
\end{gathered}
$$

where

$$
\Sigma_{8}=-\Sigma_{+} \Sigma_{-}=\left(\begin{array}{cc}
I_{4} & 0 \\
0 & -I_{4}
\end{array}\right)
$$

In what follows, we find it convenient to use the following definitions:

$$
\begin{array}{ll}
g(x)=g_{+} I_{8}+g_{-} \Sigma_{8}+g_{M} \Sigma_{M}, & g_{M}=\left\{g_{a}, g_{s}\right\}, \\
g_{ \pm}=\frac{1}{2}\left(\frac{1}{\sqrt{1-\frac{z^{2}}{4}}} \pm \frac{1}{\sqrt{1+\frac{y^{2}}{4}}}\right), & g_{a}=\frac{z_{a}}{2 \sqrt{1-\frac{z^{2}}{4}}}, \quad g_{s}=\frac{y_{s}}{2 \sqrt{1+\frac{y^{2}}{4}}} ;
\end{array}
$$




$$
\begin{aligned}
& g(x)^{2}=G_{+} I_{8}+G_{-} \Sigma_{8}+G_{M} \Sigma_{M}, \quad G_{M}=\left\{G_{a}, G_{s}\right\}, \\
& G_{ \pm}=\frac{1}{2}\left(\frac{1+\frac{z^{2}}{4}}{1-\frac{z^{2}}{4}} \pm \frac{1-\frac{y^{2}}{4}}{1+\frac{y^{2}}{4}}\right), \quad G_{a}=\frac{z_{a}}{1-\frac{z^{2}}{4}}, \quad G_{s}=\frac{y_{s}}{1+\frac{y^{2}}{4}} .
\end{aligned}
$$

Then by using (3.14) and the properties of Dirac matrices, one can easily get

$\mathbf{p}_{-}=-\frac{G_{-}}{G_{+}} P_{+}+\frac{G_{+}^{2}-G_{-}^{2}}{G_{+}} \pi_{-}-\frac{P_{+}}{4} \operatorname{Str}\left(\chi^{2}\right)+\frac{\mathrm{i}}{2} g_{N} \pi_{M} \operatorname{Str}\left(\left[\Sigma_{N}, \Sigma_{M}\right] \chi^{2}\left(\Sigma_{+} g_{+}-\Sigma_{-} g_{-}\right)\right)$.

As one can see, $\mathbf{p}_{-}$has only an explicit quadratic dependence on the fermion $\chi$. However, the true $\chi$ dependence is much more complicated because $\pi_{-}$nontrivially depends on $\chi$.

\section{B.2. Simplifying $-\operatorname{Str} \pi A_{\text {even }}^{\perp}$}

Let us introduce the even and odd components of $g^{-1}(\chi) \partial_{\alpha} g(\chi)$

$g^{-1}(\chi) \partial_{\alpha} g(\chi)=B_{\alpha}+F_{\alpha}$,

$B_{\alpha}=-\frac{1}{2} \chi \partial_{\alpha} \chi+\frac{1}{2} \partial_{\alpha} \chi \chi+\frac{1}{2} \sqrt{1+\chi^{2}} \partial_{\alpha} \sqrt{1+\chi^{2}}-\frac{1}{2} \partial_{\alpha} \sqrt{1+\chi^{2}} \sqrt{1+\chi^{2}}$,

$F_{\alpha}=\sqrt{1+\chi^{2}} \partial_{\alpha} \chi-\chi \partial_{\alpha} \sqrt{1+\chi^{2}}$.

Then $A_{\text {even }}^{\perp}$ can be written in the form

$$
A_{\text {even }}^{\perp}=-g^{-1}(x) B_{\tau} g(x)-g^{-1}(x) \partial_{\tau} g(x) .
$$

The supertrace of the second term with $\pi$ can be easily computed, and we get

$$
\operatorname{Str} \pi g^{-1}(x) \partial_{\tau} g(x)=p_{M} \partial_{\tau} x_{M},
$$

where the momenta conjugate to the coordinates $x_{M}=\left\{z_{a}, y_{s}\right\}$ are given by

$$
p_{M}=\left\{p_{a}^{z}, p_{s}^{y}\right\}, \quad p_{a}=\frac{\pi_{a}}{1-\frac{z^{2}}{4}}, \quad p_{s}=\frac{\pi_{s}}{1+\frac{y^{2}}{4}} .
$$

The term dependent on $B_{\alpha}$ can also be written in a more explicit form:

$\operatorname{Str}\left(\pi g^{-1} B_{\alpha} g\right)=\frac{\mathrm{i} \pi_{+}}{4} \operatorname{Str}\left(\Sigma_{+} B_{\alpha} g^{2}\right)+\frac{\mathrm{i} \pi_{-}}{4} \operatorname{Str}\left(\Sigma_{-} B_{\alpha} g^{2}\right)+\frac{\pi_{M}}{2} \operatorname{Str}\left(\Sigma_{M} g^{-1} B_{\alpha} g\right)$.

Now, taking into account the formulae

$\operatorname{Str}\left(\Sigma_{+} B_{\alpha}\right)=-\operatorname{Str}\left(\Sigma_{+} \chi \partial_{\alpha} \chi\right), \quad \operatorname{Str}\left(\Sigma_{-} B_{\alpha}\right)=0, \quad \operatorname{Str}\left(\Sigma_{+} \chi^{n}\right)=0$,

that follow from the fact that $\Sigma_{+}$anticommutes with $\chi, \Sigma_{-}$commutes with $\chi$ and we obtain the explicit form of $B_{\alpha}$,

$$
\operatorname{Str}\left(\pi g^{-1} B_{\alpha} g\right)=-\frac{i}{4} P_{+} \operatorname{Str}\left(\Sigma_{+} \chi \partial_{\alpha} \chi\right)+\frac{1}{2} \pi_{M} \operatorname{Str}\left(g \Sigma_{M} g^{-1} B_{\alpha}\right) .
$$

The last term in (B.10) can also be simplified by using the explicit formula (B.2) for $g(x)$, commutativity of $\Sigma_{ \pm}$with $B_{\alpha}$, and the properties of gamma matrices.

The final expression for $-\operatorname{Str} \pi A_{\text {even }}^{\perp}$ then takes the form

$-\operatorname{Str}\left(\pi A_{\text {even }}^{\perp}\right)=p_{M} \partial_{\tau} x_{M}-\frac{\mathrm{i}}{4} P_{+} \operatorname{Str}\left(\Sigma_{+} \chi \partial_{\tau} \chi\right)+\frac{1}{2} g_{N} \pi_{M} \operatorname{Str}\left(\left[\Sigma_{N}, \Sigma_{M}\right] B_{\tau}\right)$. 
B.3. Solving the Virasoro constraint $C_{2}: \operatorname{Str} \pi A_{1}^{(2)}=0$

The constraint $C_{2}=0$ can now be easily solved to find $x_{-}^{\prime} \equiv \partial_{1} x_{-}$,

$$
-\operatorname{Str}\left(\pi A_{1}^{(2)}\right)=P_{+} x_{-}^{\prime}+p_{M} x_{M}^{\prime}+\frac{\mathrm{i}}{4} P_{+} \operatorname{Str}\left(\Sigma_{+} B_{1}\right)+\frac{1}{2} \pi_{M} \operatorname{Str}\left(g \Sigma_{M} g^{-1} B_{1}\right)=0 .
$$

Thus

$x_{-}^{\prime}=-\frac{1}{P_{+}}\left(p_{M} x_{M}^{\prime}-\frac{\mathrm{i}}{4} P_{+} \operatorname{Str}\left(\Sigma_{+} \chi \chi^{\prime}\right)+\frac{1}{2} g_{N} \pi_{M} \operatorname{Str}\left(\left[\Sigma_{N}, \Sigma_{M}\right] B_{\sigma}\right)\right)$.

The nice feature is that in the light-cone gauge $x_{-}^{\prime}$ has no dependence on $\pi_{-}$.

\section{B.4. Level-matching condition}

Integrating (B.12) over $\sigma$ we derive the level-matching condition

$\mathcal{V}=\int_{0}^{2 \pi} \frac{\mathrm{d} \sigma}{2 \pi}\left(p_{M} x_{M}^{\prime}-\frac{\mathrm{i}}{4} P_{+} \operatorname{Str}\left(\Sigma_{+} \chi \chi^{\prime}\right)+\frac{1}{2} g_{N} \pi_{M} \operatorname{Str}\left(\left[\Sigma_{N}, \Sigma_{M}\right] B_{\sigma}\right)\right)=0$,

that should be imposed on physical string states.

B.5. Solving the Virasoro constraint $C_{2}: \operatorname{Str}\left(\pi^{2}+\lambda\left(A_{1}^{(2)}\right)^{2}\right)=0$

Since $x_{-}^{\prime}$ does not depend on $\pi_{-}$, the Virasoro constraint $C_{1}=0$ can be easily solved to find $\pi_{-}$. The solution has in fact the same form as in the bosonic case:

$$
\begin{aligned}
\operatorname{Str}\left(\pi^{2}+\lambda\left(A_{1}^{(2)}\right)^{2}\right) & =\pi_{+} \pi_{-}+\pi_{M}^{2}+\lambda \operatorname{Str}\left(\left(A_{1}^{(2)}\right)^{2}\right) \\
& =\frac{1}{G_{+}}\left(P_{+}+G_{-} \pi_{-}\right) \pi_{-}+\pi_{M}^{2}+\lambda \operatorname{Str}\left(\left(A_{1}^{(2)}\right)^{2}\right)=0 .
\end{aligned}
$$

Let us denote

$$
\mathcal{A}^{2} \equiv \operatorname{Str}\left(\left(A_{1}^{(2)}\right)^{2}\right)
$$

to simplify the notation. Then the solution to this constraint is

$$
\pi_{-}=-\frac{2 G_{+}\left(\pi_{M}^{2}+\lambda \mathcal{A}^{2}\right)}{P_{+}+\sqrt{P_{+}^{2}-4 G_{+} G_{-}\left(\pi_{M}^{2}+\lambda \mathcal{A}^{2}\right)}} .
$$

\section{B.6. Preliminary form of the gauge-fixed Lagrangian}

The gauge-fixed Lagrangian can now be written in the form

$\mathscr{L}_{g f}=p_{M} \dot{x}_{M}-\frac{\mathrm{i} P_{+}}{4} \operatorname{Str}\left(\Sigma_{+} \chi \partial_{\tau} \chi\right)+\frac{1}{2} g_{N} \pi_{M} \operatorname{Str}\left(\left[\Sigma_{N}, \Sigma_{M}\right] B_{\tau}\right)+\mathscr{L}_{\mathrm{WZ}}+\mathbf{p}_{-}$.

Here we should use the formulae for $\pi_{-}, x_{-}^{\prime}$ and $\mathbf{p}_{-}$to express everything in terms of physical fields.

\section{B.7. Simplifying $\mathcal{A}^{2}$}

To compute $\mathcal{A}^{2}$ we use the following formula:

$A_{1}^{(2)}=-\left(\frac{\mathrm{i}}{4} x_{-}^{\prime}\left(\Sigma_{-} g^{2}+g^{2} \Sigma_{-}\right)+\frac{1}{2}\left(g^{-1} B_{1} g-g K_{8} B_{1}^{t} K_{8} g^{-1}\right)+\frac{1}{2}\left(g^{-1} g^{\prime}+g^{\prime} g^{-1}\right)\right)$.

We see that $\mathcal{A}^{2}$ is given by the sum of three terms: (i) quadratic in $x_{-}^{\prime}$, (ii) linear in $x_{-}^{\prime}$, and (iii) independent of $x_{-}^{\prime}$. 
B.7.1. Computing the term quadratic in $x_{-}^{\prime}$. This term is equal to

$$
-\frac{x_{-}^{\prime 2}}{16} \operatorname{Str}\left[\left(\Sigma_{-} g^{2}+g^{2} \Sigma_{-}\right)\left(\Sigma_{-} g^{2}+g^{2} \Sigma_{-}\right)\right]=-4 x_{-}^{\prime 2} G_{+} G_{-},
$$

where we took into account that $\Sigma_{-} g=g^{-1} \Sigma_{-}, \Sigma_{-}^{2}=I_{8}$, and the formula

$$
g^{4}+g^{-4}=2\left(G_{t t}+G_{\phi \phi}-1\right) I_{8}+8 G_{+} G_{-} \Sigma_{8}
$$

B.7.2. Computing the term linear in $x_{-}^{\prime}$. The linear term is equal to

$$
\begin{gathered}
\frac{\mathrm{i}}{4} x_{-}^{\prime} \operatorname{Str}\left[\left(\Sigma_{-} g^{2}+g^{2} \Sigma_{-}\right)\left(g^{-1} B_{1} g-g K_{8} B_{1}^{t} K_{8} g^{-1}+g^{-1} g^{\prime}+g^{\prime} g^{-1}\right)\right] \\
=2 \mathrm{i} x_{-}^{\prime} G_{+} G_{-} \operatorname{Str}\left(\Sigma_{+} \chi \chi^{\prime}\right),
\end{gathered}
$$

where we used that $\operatorname{Str} \Sigma_{-} g^{n} g^{\prime}=0$ for any $n$, and equation (B.20).

B.7.3. Simplifying the term independent of $x_{-}^{\prime}$. The term independent of $x_{-}^{\prime}$ is

$$
\begin{aligned}
\frac{1}{4} \operatorname{Str}\left[\left(g^{-1} B_{1} g\right.\right. & \left.\left.-g K_{8} B_{1}^{t} K_{8} g^{-1}+g^{-1} g^{\prime}+g^{\prime} g^{-1}\right)^{2}\right] \\
= & \frac{1}{4} \operatorname{Str}\left[\left(g^{-1} B_{1} g-g K_{8} B_{1}^{t} K_{8} g^{-1}\right)^{2}\right] \\
& +\frac{1}{2} \operatorname{Str}\left[\left(g^{-1} B_{1} g-g K_{8} B_{1}^{t} K_{8} g^{-1}\right)\left(g^{-1} g^{\prime}+g^{\prime} g^{-1}\right)\right] \\
& +\frac{1}{4} \operatorname{Str}\left[\left(g^{-1} g^{\prime}+g^{\prime} g^{-1}\right)^{2}\right] .
\end{aligned}
$$

The last term independent of $B_{1}$ in (B.22) can be easily computed,

$$
\frac{1}{4} \operatorname{Str}\left[\left(g^{-1} g^{\prime}+g^{\prime} g^{-1}\right)^{2}\right]=\frac{z_{a}^{\prime 2}}{\left(1-\frac{z^{2}}{4}\right)^{2}}+\frac{y_{s}^{\prime 2}}{\left(1+\frac{y^{2}}{4}\right)^{2}} .
$$

The first term in (B.22) can be cast in the form

$\frac{1}{4} \operatorname{Str}\left[\left(g^{-1} B_{1} g-g K_{8} B_{1}^{t} K_{8} g^{-1}\right)^{2}\right]=\frac{1}{2} \operatorname{Str} B_{1}^{2}-\frac{1}{2} \operatorname{Str}\left(g^{-2} B_{1} g^{2} K_{8} B_{1}^{t} K_{8}\right)$.

Note that this term is of the fourth order in fermions. The second term in (B.24) can be written in a more explicit form

$$
\begin{aligned}
& -\frac{1}{2} \operatorname{Str}\left(g^{-2} B_{1} g^{2} K_{8} B_{1}^{t} K_{8}\right)=-\frac{1}{2}\left(G_{+}^{2}+G_{-}^{2}\right) \operatorname{Str}\left(B_{1} K_{8} B_{1}^{t} K_{8}\right) \\
& -G_{+} G_{-} \operatorname{Str}\left(\Sigma_{8} B_{1} K_{8} B_{1}^{t} K_{8}\right)+\frac{1}{2} G_{M} G_{N} \operatorname{Str}\left(\Sigma_{M} B_{1} \Sigma_{N} K_{8} B_{1}^{t} K_{8}\right) .
\end{aligned}
$$

The second term in (B.22) can be cast in the form

$$
\begin{gathered}
\frac{1}{2} \operatorname{Str}\left[\left(g^{-1} B_{1} g-g K_{8} B_{1}^{t} K_{8} g^{-1}\right)\left(g^{-1} g^{\prime}+g^{\prime} g^{-1}\right)\right]=\operatorname{Str}\left[B_{1}\left(g^{2}\right)^{\prime} g^{-2}\right] \\
=\left(G_{+} G_{-}\right)^{\prime} \operatorname{Str}\left(\Sigma_{8} B_{1}\right)-G_{M}^{\prime} G_{N} \operatorname{Str}\left(\Sigma_{M} \Sigma_{N} B_{1}\right) \\
=-\frac{1}{2} G_{M}^{\prime} G_{N} \operatorname{Str}\left(\left[\Sigma_{M}, \Sigma_{N}\right] B_{1}\right)
\end{gathered}
$$

where we took into account that $2 G_{+} G_{-} \Sigma_{8}-G_{M} G_{N} \Sigma_{M} \Sigma_{N}+\left(G_{+}^{2}+G_{-}^{2}-1\right) I_{8}=0$. 
B.7.4. Final form of $\mathcal{A}^{2}$. Collecting the pieces together we get $\mathcal{A}^{2}$

$$
\begin{aligned}
\mathcal{A}^{2}=\operatorname{Str}\left[\left(A_{1}^{(2)}\right)^{2}\right]= & -4 x_{-}^{\prime 2} G_{+} G_{-}+2 \mathrm{i} x_{-}^{\prime} G_{+} G_{-} \operatorname{Str}\left(\Sigma_{+} \chi \chi^{\prime}\right)+\frac{z_{a}^{\prime 2}}{\left(1-\frac{z^{2}}{4}\right)^{2}}+\frac{y_{s}^{\prime 2}}{\left(1+\frac{y^{2}}{4}\right)^{2}} \\
& -\frac{1}{2} G_{M}^{\prime} G_{N} \operatorname{Str}\left(\left[\Sigma_{M}, \Sigma_{N}\right] B_{1}\right)+\frac{1}{2} \operatorname{Str} B_{1}^{2}-\frac{1}{2} \operatorname{Str}\left(g^{-2} B_{1} g^{2} K_{8} B_{1}^{t} K_{8}\right) \\
= & -4 x_{-}^{\prime 2} G_{+} G_{-}+2 \mathrm{i} x_{-}^{\prime} G_{+} G_{-} \operatorname{Str}\left(\Sigma_{+} \chi \chi^{\prime}\right)+\frac{z_{a}^{\prime 2}}{\left(1-\frac{z^{2}}{4}\right)^{2}}+\frac{y_{s}^{\prime 2}}{\left(1+\frac{y^{2}}{4}\right)^{2}} \\
& -\frac{1}{2} G_{M}^{\prime} G_{N} \operatorname{Str}\left(\left[\Sigma_{M}, \Sigma_{N}\right] B_{1}\right)+\frac{1}{2} \operatorname{Str} B_{1}^{2}-\frac{1}{2}\left(G_{+}^{2}+G_{-}^{2}\right) \operatorname{Str}\left(B_{1} K_{8} B_{1}^{t} K_{8}\right) \\
& -G_{+} G_{-} \operatorname{Str}\left(\Sigma_{8} B_{1} K_{8} B_{1}^{t} K_{8}\right)+\frac{1}{2} G_{M} G_{N} \operatorname{Str}\left(\Sigma_{M} B_{1} \Sigma_{N} K_{8} B_{1}^{t} K_{8}\right) .
\end{aligned}
$$

These formulae allow us to compute all necessary terms very efficiently.

\section{B.8. Simplifying the Wess-Zumino term}

By using the decomposition (B.5), the odd components of $A_{\alpha}$ can be written in the form

$$
\begin{aligned}
& A_{\tau}^{\text {odd }}=-\mathrm{i}^{-1}(x) \Sigma_{+} \chi \sqrt{1+\chi^{2}} g(x)-g^{-1}(x) F_{\tau} g(x), \\
& A_{\sigma}^{\text {odd }}=-g^{-1}(x) F_{\sigma} g(x) .
\end{aligned}
$$

Then the Wess-Zumino term can be written as a sum of the two terms,

$$
\begin{gathered}
\mathscr{L}_{\mathrm{WZ}}=-\kappa \frac{\sqrt{\lambda}}{2} \epsilon^{\alpha \beta} \operatorname{Str} A_{\alpha}^{(1)} A_{\beta}^{(3)}=\mathrm{i} \kappa \frac{\sqrt{\lambda}}{2} \operatorname{Str} F_{\tau} g(x)^{2} \widetilde{K}_{8} F_{\sigma}^{t} K_{8} g(x)^{-2} \\
-\kappa \frac{\sqrt{\lambda}}{2} \operatorname{Str} \Sigma_{+} \chi \sqrt{1+\chi^{2}} g(x)^{2} \widetilde{K}_{8} F_{\sigma}^{t} K_{8} g(x)^{-2} .
\end{gathered}
$$

It is clear that the second term in (B.30) represents the additional contribution of the WessZumino term to the momentum $p_{-}$canonically conjugate to $x_{+}$.

Both terms can be written in the following more explicit form by using expression (B.3) for $g^{2}$,

$$
\begin{gathered}
\mathrm{i} \kappa \frac{\sqrt{\lambda}}{2} \operatorname{Str} F_{\tau} g(x)^{2} \widetilde{K}_{8} F_{\sigma}^{t} K_{8} g(x)^{-2}=\mathrm{i} \kappa \frac{\sqrt{\lambda}}{2}\left(G_{+}^{2}-G_{-}^{2}\right) \operatorname{Str}\left(F_{\tau} \widetilde{K}_{8} F_{\sigma}^{t} K_{8}\right) \\
-\mathrm{i} \kappa \frac{\sqrt{\lambda}}{2} G_{M} G_{N} \operatorname{Str}\left(\Sigma_{N} F_{\tau} \Sigma_{M} \widetilde{K}_{8} F_{\sigma}^{t} K_{8}\right) \\
-\kappa \frac{\sqrt{\lambda}}{2} \operatorname{Str} \Sigma_{+} \chi \sqrt{1+\chi^{2}} g(x)^{2} \widetilde{K}_{8} F_{\sigma}^{t} K_{8} g(x)^{-2} \\
=-\kappa \frac{\sqrt{\lambda}}{2}\left(G_{+}^{2}-G_{-}^{2}\right) \operatorname{Str}\left(\Sigma_{+} \chi \sqrt{1+\chi^{2}} \widetilde{K}_{8} F_{\sigma}^{t} K_{8}\right) \\
-\kappa \frac{\sqrt{\lambda}}{2} G_{M} G_{N} \operatorname{Str}\left(\Sigma_{+} \Sigma_{N} \chi \sqrt{1+\chi^{2}} \Sigma_{M} \widetilde{K}_{8} F_{\sigma}^{t} K_{8}\right)
\end{gathered}
$$

The Wess-Zumino term is given by the sum of the terms in (B.31) and (B.32). 


\section{B.9. Final form of the gauge-fixed Lagrangian}

It is useful to single out a kinetic term and the density of the Hamiltonian from the gauge-fixed Lagrangian (B.17), and write it in the form

$$
\mathscr{L}_{\text {gf }}=\mathscr{L}_{\text {kin }}-\mathcal{H}
$$

where

$$
\begin{aligned}
\mathscr{L}_{\text {kin }}=p_{M} \dot{x}_{M} & -\frac{\mathrm{i} P_{+}}{4} \operatorname{Str}\left(\Sigma_{+} \chi \partial_{\tau} \chi\right)+\frac{1}{2} g_{N} \pi_{M} \operatorname{Str}\left(\left[\Sigma_{N}, \Sigma_{M}\right] B_{\tau}\right) \\
& +\mathrm{i} \kappa \frac{\sqrt{\lambda}}{2}\left(G_{+}^{2}-G_{-}^{2}\right) \operatorname{Str}\left(F_{\tau} \widetilde{K}_{8} F_{\sigma}^{t} K_{8}\right) \\
& -\mathrm{i} \kappa \frac{\sqrt{\lambda}}{2} G_{M} G_{N} \operatorname{Str}\left(\Sigma_{N} F_{\tau} \Sigma_{M} \widetilde{K}_{8} F_{\sigma}^{t} K_{8}\right), \\
\mathcal{H}=-\mathbf{p}_{-}+\kappa & \frac{\sqrt{\lambda}}{2}\left(G_{+}^{2}-G_{-}^{2}\right) \operatorname{Str}\left(\Sigma_{+} \chi \sqrt{1+\chi^{2}} \widetilde{K}_{8} F_{\sigma}^{t} K_{8}\right) \\
+ & \kappa \frac{\sqrt{\lambda}}{2} G_{M} G_{N} \operatorname{Str}\left(\Sigma_{+} \Sigma_{N} \chi \sqrt{1+\chi^{2}} \Sigma_{M} \widetilde{K}_{8} F_{\sigma}^{t} K_{8}\right) .
\end{aligned}
$$

Here $\mathbf{p}_{-}$is given by (B.4), and we should use the formulae (B.16), (B.12) and (B.27) for $\pi_{-}, x_{-}^{\prime}$ and $\mathcal{A}^{2}$ to express everything in terms of physical fields.

\section{Appendix C. Deriving the quartic Hamiltonian}

In this appendix, we derive the various forms of the quartic Hamiltonian used in the paper.

\section{C.1. Redefining fermions}

The kinetic part (B.34) of the gauge-fixed Lagrangian (B.33) up to the quartic order in fermions can be written in the form $(5.1)$ where $\Phi(p, x, \chi)$ is given by

$$
\begin{aligned}
\Phi(p, x, \chi)= & -\frac{1}{p_{+}} \frac{\mathrm{i}}{2} g_{N} \pi_{M} \Sigma_{+}\left[\chi,\left[\Sigma_{N}, \Sigma_{M}\right]\right] \\
& +\frac{1}{p_{+}} \kappa \sqrt{\lambda} \Sigma_{+}\left[-\frac{1}{2} \widetilde{K}_{8}\left(\chi \chi^{\prime} \chi\right)^{t} K_{8}-\frac{1}{2} \chi \widetilde{K}_{8} \chi^{\prime t} K_{8} \chi\right. \\
& \left.+\left(G_{+}^{2}-G_{-}^{2}-1\right) \widetilde{K}_{8} \chi^{\prime t} K_{8}-G_{M} G_{N} \Sigma_{M} \widetilde{K}_{8} \chi^{\prime t} K_{8} \Sigma_{N}\right]
\end{aligned}
$$

Expanding the bosonic fields, and keeping only terms quartic in fields, we get

$$
\begin{gathered}
\Phi=-\frac{1}{p_{+}} \frac{\mathrm{i}}{4} x_{N} p_{M} \Sigma_{+}\left[\chi,\left[\Sigma_{N}, \Sigma_{M}\right]\right]+\frac{1}{p_{+}} \kappa \sqrt{\lambda} \Sigma_{+}\left[-\frac{1}{2} \widetilde{K}_{8}\left(\chi \chi^{\prime} \chi\right)^{t} K_{8}-\frac{1}{2} \chi \widetilde{K}_{8} \chi^{\prime t} K_{8} \chi\right. \\
\left.+\frac{1}{2}\left(z^{2}-y^{2}\right) \widetilde{K}_{8} \chi^{\prime t} K_{8}-x_{M} x_{N} \Sigma_{M} \widetilde{K}_{8} \chi^{\prime t} K_{8} \Sigma_{N}\right] .
\end{gathered}
$$

Then the redefinition of $\chi$ is

$$
\chi \rightarrow \chi+\Phi(p, x, \chi)
$$

These shifts of fermions produce additional quartic terms in the Hamiltonian but all these terms come only from its fermionic quadratic part

$$
\mathcal{H}_{2}^{\text {ferm }}=\frac{\kappa}{2} \sqrt{\lambda} \operatorname{Str}\left(\Sigma_{+} \chi \widetilde{K}_{8} \chi^{\prime t} K_{8}\right)+\frac{p_{+}}{4} \operatorname{Str} \chi^{2} .
$$


The additional quartic terms in the Hamiltonian are equal to

$$
H_{\text {add }}=\kappa \sqrt{\lambda} \operatorname{Str}\left(\Sigma_{+} \Phi \widetilde{K}_{8} \chi^{\prime t} K_{8}\right)+\frac{p_{+}}{2} \operatorname{Str} \Phi \chi .
$$

The first term in (C.5) gives

$$
\begin{gathered}
\kappa \sqrt{\lambda} \operatorname{Str}\left(\Sigma_{+} \Phi \widetilde{K}_{8} \chi^{\prime t} K_{8}\right)=\frac{\mathrm{i} \kappa \sqrt{\lambda}}{8 p_{+}}\left(x_{N} p_{M}\right)^{\prime} \operatorname{Str}\left(\left[\Sigma_{N}, \Sigma_{M}\right]\left(\widetilde{K}_{8} \chi^{t} K_{8} \chi-\chi \widetilde{K}_{8} \chi^{t} K_{8}\right)\right) \\
-\frac{\lambda}{2 p_{+}} \operatorname{Str}\left(\chi \chi^{\prime} \chi \chi^{\prime}\right)-\frac{\lambda}{2 p_{+}} \operatorname{Str}\left(\chi \widetilde{K}_{8} \chi^{\prime t} K_{8} \chi \widetilde{K}_{8} \chi^{\prime t} K_{8}\right) \\
+\frac{\lambda}{2 p_{+}}\left(z^{2}-y^{2}\right) \operatorname{Str}\left(\chi^{\prime} \chi^{\prime}\right)-\frac{\lambda}{p_{+}} x_{M} x_{N} \operatorname{Str}\left(\Sigma_{M} \chi^{\prime} \Sigma_{N} \chi^{\prime}\right) .
\end{gathered}
$$

Computing the second term we get

$$
\begin{aligned}
\frac{p_{+}}{2} \operatorname{Str} \Phi \chi= & \frac{\mathrm{i}}{4} x_{N} p_{M} \operatorname{Str} \Sigma_{+}\left[\Sigma_{N}, \Sigma_{M}\right] \chi^{2}-\frac{\kappa}{2} \sqrt{\lambda} \operatorname{Str}\left(\frac{1}{2}\left(z^{2}-y^{2}\right) \Sigma_{+} \chi \widetilde{K}_{8} \chi^{\prime t} K_{8}\right. \\
& \left.+\frac{1}{2} \Sigma_{+} \chi^{3} \widetilde{K}_{8} \chi^{\prime t} K_{8}+\frac{1}{2} \Sigma_{+} \chi \chi^{\prime} \chi \widetilde{K}_{8} \chi^{t} K_{8}+x_{M} x_{N} \Sigma_{+} \Sigma_{N} \chi \Sigma_{M} \widetilde{K}_{8} \chi^{\prime t} K_{8}\right) .
\end{aligned}
$$

We will see that the first term cancels the same term coming from $p_{-}$, and the terms with $\kappa$ just cancel all quartic terms in $\mathcal{H}_{\mathrm{WZ}}$ (4.5).

C.2. $-\mathbf{p}_{-}$up to the quartic order

$-\mathbf{p}_{-}$(B.4) is given by

$$
-\mathbf{p}_{-}=\frac{G_{-}}{G_{+}} P_{+}-\frac{G_{+}^{2}-G_{-}^{2}}{G_{+}} \pi_{-}+\frac{P_{+}}{4} \operatorname{Str}\left(\chi^{2}\right)-\frac{\mathrm{i}}{2} g_{N} \pi_{M} \operatorname{Str}\left(\left[\Sigma_{N}, \Sigma_{M}\right] \chi^{2}\left(\Sigma_{+} g_{+}-\Sigma_{-} g_{-}\right)\right) .
$$

C.2.1. $\frac{G_{-}}{G_{+}} P_{+}$up to the sixth-order term.

$$
\frac{G_{-}}{G_{+}} P_{+} \approx \frac{P_{+}}{4}\left(y^{2}+z^{2}\right)-\frac{1}{64} P_{+} y^{2} z^{2}\left(y^{2}+z^{2}\right) .
$$

After rescaling (5.12) it takes the form

$$
\frac{G_{-}}{G_{+}} P_{+} \approx \frac{1}{2}\left(y^{2}+z^{2}\right)-\frac{1}{8 P_{+}^{2}} y^{2} z^{2}\left(y^{2}+z^{2}\right) .
$$

We see that it does not have a quartic term.

C.2.2. $\frac{G_{+}^{2}-G_{-}^{2}}{G_{+}} \pi_{-}$up to the quartic order. Since $G_{-}^{2}$ is of the quartic order and $\pi_{-}$is of the quadratic order, we get

$$
-\frac{G_{+}^{2}-G_{-}^{2}}{G_{+}} \pi_{-} \approx-G_{+} \pi_{-} \approx \frac{1}{P_{+}} G_{+}^{2}\left(\pi_{M}^{2}+\lambda \mathcal{A}^{2}\right)
$$

where (see (B.27))

$$
\begin{gathered}
\mathcal{A}^{2} \approx \frac{z^{\prime 2}}{\left(1-\frac{z^{2}}{4}\right)^{2}}+\frac{y^{\prime 2}}{\left(1+\frac{y^{2}}{4}\right)^{2}}+\frac{1}{2} \operatorname{Str} B_{1}^{2}-\frac{1}{2} \operatorname{Str}\left(B_{1} K_{8} B_{1}^{t} K_{8}\right) \\
+\frac{1}{4} x_{M}^{\prime} x_{N} \operatorname{Str}\left(\left[\Sigma_{M}, \Sigma_{N}\right]\left(\chi \chi^{\prime}-\chi^{\prime} \chi\right)\right) .
\end{gathered}
$$


We also have

$$
\begin{aligned}
& \operatorname{Str} B_{1}^{2}=\frac{1}{2} \operatorname{Str}\left(\chi \chi^{\prime} \chi \chi^{\prime}-\chi^{2} \chi^{\prime 2}\right) \\
& \operatorname{Str}\left(B_{1} K_{8} B_{1}^{t} K_{8}\right)=\frac{1}{4} \operatorname{Str}\left(\left(\chi \chi^{\prime}-\chi^{\prime} \chi\right) K_{8}\left(\chi \chi^{\prime}-\chi^{\prime} \chi\right)^{t} K_{8}\right)
\end{aligned}
$$

Then we get

$$
\begin{aligned}
\mathcal{A}^{2} \approx z^{\prime 2}+y^{\prime 2} & +\frac{1}{2} z^{\prime 2} z^{2}-\frac{1}{2} y^{\prime 2} y^{2}+\frac{1}{4} x_{M}^{\prime} x_{N} \operatorname{Str}\left(\left[\Sigma_{M}, \Sigma_{N}\right]\left(\chi \chi^{\prime}-\chi^{\prime} \chi\right)\right) \\
& +\frac{1}{4} \operatorname{Str}\left(\chi \chi^{\prime} \chi \chi^{\prime}-\chi^{2} \chi^{\prime 2}\right)-\frac{1}{8} \operatorname{Str}\left(\left(\chi \chi^{\prime}-\chi^{\prime} \chi\right) K_{8}\left(\chi \chi^{\prime}-\chi^{\prime} \chi\right)^{t} K_{8}\right) .
\end{aligned}
$$

The expansion of $G_{+}^{2}$ gives

$$
G_{+}^{2} \approx 1+\frac{1}{2}\left(z^{2}-y^{2}\right)
$$

so we have

$$
\begin{aligned}
-\frac{G_{+}^{2}-G_{-}^{2}}{G_{+}} \pi_{-} & \approx \frac{1}{P_{+}}\left(p_{z}^{2}+p_{y}^{2}+\lambda\left(z^{\prime 2}+y^{\prime 2}\right)+\frac{1}{2}\left(p_{y}^{2} z^{2}-p_{z}^{2} y^{2}\right)\right. \\
+ & \frac{\lambda}{2}\left(y^{\prime 2} z^{2}-z^{\prime 2} y^{2}\right)+\lambda\left(z^{\prime 2} z^{2}-y^{\prime 2} y^{2}\right) \\
& +\frac{1}{4} \lambda x_{M}^{\prime} x_{N} \operatorname{Str}\left(\left[\Sigma_{M}, \Sigma_{N}\right]\left(\chi \chi^{\prime}-\chi^{\prime} \chi\right)\right)+\frac{\lambda}{4} \operatorname{Str}\left(\chi \chi^{\prime} \chi \chi^{\prime}-\chi^{2} \chi^{\prime 2}\right) \\
& \left.-\frac{\lambda}{8} \operatorname{Str}\left(\left(\chi \chi^{\prime}-\chi^{\prime} \chi\right) K_{8}\left(\chi \chi^{\prime}-\chi^{\prime} \chi\right)^{t} K_{8}\right)\right) .
\end{aligned}
$$

C.2.3. The last term of $-\mathbf{p}_{-}$up to the quartic order. For the last term in (C.8) we find $-\frac{\mathrm{i}}{2} g_{N} \pi_{M} \operatorname{Str}\left[\Sigma_{N}, \Sigma_{M}\right] \chi^{2}\left(\Sigma_{+} g_{+}-\Sigma_{-} g_{-}\right) \approx-\frac{\mathrm{i}}{4} x_{N} p_{M} \operatorname{Str} \Sigma_{+}\left[\Sigma_{N}, \Sigma_{M}\right] \chi^{2}$.

We see that this term is cancelled by the first term in (C.7).

C.2.4. The final result for $-\mathbf{p}_{-}$up to the quartic order. Summing up the terms we get for $-\mathbf{p}_{-}$

$$
\begin{aligned}
-\mathbf{p}_{-} \approx \frac{1}{4} P_{+} & \left(y^{2}+z^{2}+\operatorname{Str}\left(\chi^{2}\right)\right)+\frac{1}{P_{+}}\left(p_{z}^{2}+p_{y}^{2}+\lambda\left(z^{\prime 2}+y^{\prime 2}\right)\right. \\
& +\frac{1}{2}\left(p_{y}^{2} z^{2}-p_{z}^{2} y^{2}\right)+\frac{\lambda}{2}\left(y^{\prime 2} z^{2}-z^{\prime 2} y^{2}\right)+\lambda\left(z^{\prime 2} z^{2}-y^{\prime 2} y^{2}\right) \\
& +\frac{\lambda}{4} x_{M}^{\prime} x_{N} \operatorname{Str}\left(\left[\Sigma_{M}, \Sigma_{N}\right]\left(\chi \chi^{\prime}-\chi^{\prime} \chi\right)\right) \\
& \left.+\frac{\lambda}{4} \operatorname{Str}\left(\chi \chi^{\prime} \chi \chi^{\prime}-\chi^{2} \chi^{\prime 2}\right)-\frac{\lambda}{8} \operatorname{Str}\left(\left(\chi \chi^{\prime}-\chi^{\prime} \chi\right) K_{8}\left(\chi \chi^{\prime}-\chi^{\prime} \chi\right)^{t} K_{8}\right)\right) \\
& -\frac{\mathrm{i}}{4} x_{N} p_{M} \operatorname{Str} \Sigma_{+}\left[\Sigma_{N}, \Sigma_{M}\right] \chi^{2} .
\end{aligned}
$$

After rescaling according to (5.12) this takes the form

$$
\begin{aligned}
-\mathbf{p}_{-} \approx \frac{1}{2}\left(p_{z}^{2}+\right. & \left.p_{y}^{2}+z^{2}+y^{2}+\tilde{\lambda}\left(z^{\prime 2}+y^{\prime 2}\right)+\operatorname{Str}\left(\chi^{2}\right)\right) \\
& +\frac{1}{2 P_{+}}\left(p_{y}^{2} z^{2}-p_{z}^{2} y^{2}+\tilde{\lambda}\left(y^{\prime 2} z^{2}-z^{\prime 2} y^{2}\right)+2 \tilde{\lambda}\left(z^{\prime 2} z^{2}-y^{\prime 2} y^{2}\right)\right. \\
& +\frac{\tilde{\lambda}}{2} x_{M}^{\prime} x_{N} \operatorname{Str}\left(\left[\Sigma_{M}, \Sigma_{N}\right]\left(\chi \chi^{\prime}-\chi^{\prime} \chi\right)\right)+\frac{\tilde{\lambda}}{2} \operatorname{Str}\left(\chi \chi^{\prime} \chi \chi^{\prime}-\chi^{2} \chi^{\prime 2}\right) \\
& \left.-\frac{\tilde{\lambda}}{4} \operatorname{Str}\left(\left(\chi \chi^{\prime}-\chi^{\prime} \chi\right) K_{8}\left(\chi \chi^{\prime}-\chi^{\prime} \chi\right)^{t} K_{8}\right)-\mathrm{i} x_{N} p_{M} \operatorname{Str} \Sigma_{+}\left[\Sigma_{N}, \Sigma_{M}\right] \chi^{2}\right) .
\end{aligned}
$$




\section{C.3. Contribution of the WZ term to the Hamiltonian}

Contribution of the Wess-Zumino term to the Hamiltonian (B.35) is given by

$H_{\mathrm{WZ}}=\frac{\kappa}{2} \sqrt{\lambda} \operatorname{Str}\left(\left(G_{+}^{2}-G_{-}^{2}\right) \Sigma_{+} \chi \sqrt{1+\chi^{2}} \widetilde{K}_{8} F_{1}^{t} K_{8}+G_{M} G_{N} \Sigma_{+} \Sigma_{N} \chi \sqrt{1+\chi^{2}} \Sigma_{M} \widetilde{K}_{8} F_{1}^{t} K_{8}\right)$.

Up to the quartic order we get

$$
\begin{aligned}
H_{\mathrm{WZ}}=\frac{\kappa}{2} \sqrt{\lambda} & \operatorname{Str}\left(\Sigma_{+} \chi \widetilde{K}_{8} \chi^{\prime t} K_{8}+\frac{1}{2}\left(z^{2}-y^{2}\right) \Sigma_{+} \chi \widetilde{K}_{8} \chi^{\prime t} K_{8}+\frac{1}{2} \Sigma_{+} \chi^{3} \widetilde{K}_{8} \chi^{\prime t} K_{8}\right. \\
& \left.+\frac{1}{2} \Sigma_{+} \chi \chi^{\prime} \chi \widetilde{K}_{8} \chi^{t} K_{8}+x_{M} x_{N} \Sigma_{+} \Sigma_{N} \chi \Sigma_{M} \widetilde{K}_{8} \chi^{\prime t} K_{8}\right) .
\end{aligned}
$$

We see that it is exactly cancelled by the contribution (C.7) coming from the fermion shift (C.3).

\section{C.4. Quartic Hamiltonian in terms of $\chi$}

Summing up all the contributions we get the quartic Hamiltonian

$$
\begin{aligned}
\mathcal{H}_{4}=\frac{1}{2 P_{+}}[ & p_{y}^{2} z^{2}-p_{z}^{2} y^{2}+\tilde{\lambda}\left(y^{\prime 2} z^{2}-z^{\prime 2} y^{2}\right)+2 \tilde{\lambda}\left(z^{\prime 2} z^{2}-y^{\prime 2} y^{2}\right)-\tilde{\lambda} \operatorname{Str}\left(\frac{1}{2} \chi \chi^{\prime} \chi \chi^{\prime}+\chi^{2} \chi^{\prime 2}\right. \\
& \left.+\frac{1}{4}\left(\chi \chi^{\prime}-\chi^{\prime} \chi\right) K_{8}\left(\chi \chi^{\prime}-\chi^{\prime} \chi\right)^{t} K_{8}+\chi \widetilde{K}_{8} \chi^{\prime t} K_{8} \chi \widetilde{K}_{8} \chi^{\prime t} K_{8}\right) \\
& +\tilde{\lambda} \operatorname{Str}\left(\left(z^{2}-y^{2}\right) \chi^{\prime} \chi^{\prime}+\frac{1}{2} x_{M}^{\prime} x_{N}\left[\Sigma_{M}, \Sigma_{N}\right]\left(\chi \chi^{\prime}-\chi^{\prime} \chi\right)-2 x_{M} x_{N} \Sigma_{M} \chi^{\prime} \Sigma_{N} \chi^{\prime}\right) \\
& \left.+\frac{\mathrm{i} \kappa \sqrt{\hat{\lambda}}}{4}\left(x_{N} p_{M}\right)^{\prime} \operatorname{Str}\left(\left[\Sigma_{N}, \Sigma_{M}\right]\left(\widetilde{K}_{8} \chi^{t} K_{8} \chi-\chi \widetilde{K}_{8} \chi^{t} K_{8}\right)\right)\right] .
\end{aligned}
$$

The bosonic part of the Hamiltonian can be further simplified if we consider the point-particle reduction of the Hamiltonian, that is, if we assume the fields to be independent of $\sigma$, we get

$$
\mathcal{H}_{\text {particle }}=\frac{p_{M}^{2}}{2}+\frac{x_{M}^{2}}{2}+\frac{1}{2} \operatorname{tr}\left(\eta^{\dagger} \eta+\theta^{\dagger} \theta\right)+\frac{1}{2 P_{+}}\left(p_{y}^{2} z^{2}-p_{z}^{2} y^{2}\right) .
$$

We see first of all that the fermionic part of the Hamiltonian is just given by the quadratic term. Moreover, the bosonic quartic term can be removed from the Hamiltonian (C.23) by means of the canonical transformation generated by

$$
V=\frac{1}{2 P_{+}}\left(p_{y} y z^{2}-p_{z} z y^{2}\right)
$$

Let us recall that given a generating function $V(p, x)$, the canonical transformation of an arbitrary function $f(p, x)$ of the phase space can be written in the form

$$
\begin{aligned}
f(p, x) \rightarrow \tilde{f}(p, x) & =f(p, x)+\{V(p, x), f(p, x)\}+\frac{1}{2}\{V,\{V, f\}\}+\cdots \\
& =f+\sum_{n=1}^{\infty} \frac{1}{n !} \underbrace{\{V,\{V, \cdots\{V, f\} \cdots\},}_{n}
\end{aligned}
$$

where the Poisson bracket is defined as

$$
\{V(p, x), f(p, x)\}=\frac{\partial V(p, x)}{\partial p_{M}} \frac{\partial f(p, x)}{\partial x_{M}}-\frac{\partial V(p, x)}{\partial x_{M}} \frac{\partial f(p, x)}{\partial p_{M}},
$$


that is,

$$
\left\{p_{M}, x_{N}\right\}=\delta_{M N}
$$

In quantum theory, the canonical transformation corresponds to a unitary transformation generated by the operator $U$

$U=\mathrm{e}^{\mathrm{i} V}, \quad f(p, x) \rightarrow \tilde{f}(p, x)=U f(p, x) U^{\dagger}, \quad[p, x]=\frac{1}{\mathrm{i}}$.

The canonical transformation (C.24) can be easily lifted to $\sigma$-dependent fields

$$
V=\frac{1}{2 P_{+}} \int_{0}^{2 \pi} \frac{\mathrm{d} \sigma}{2 \pi}\left(p_{y} y z^{2}-p_{z} z y^{2}\right) .
$$

Then by using (C.25) one can easily find that the bosonic part of the canonically transformed Hamiltonian takes the form (5.22)

$$
\mathcal{H}_{b b}=\frac{\tilde{\lambda}}{p_{+}}\left(y^{\prime 2} z^{2}-y^{2} z^{\prime 2}+z^{2} z^{\prime 2}-y^{2} y^{\prime 2}\right),
$$

and we get (5.16).

The canonical transformation (C.25) removes all non-derivative terms from the Hamiltonian. One can show that the sixth-order non-derivative terms can also be removed by a canonical transformation. This is in accord with the observation that already the quadratic particle Hamiltonian reproduces the spectrum of type IIB supergravity on $\operatorname{AdS}_{5} \times \mathrm{S}^{5}$.

\section{Appendix D. Hamiltonian in terms of $\eta$ and $\theta$}

In this appendix, we use the decomposition (5.5) for $\chi$ to express (5.16) in terms of $\eta$ and $\theta$.

Quadratic Hamiltonian. We first use

$$
\begin{aligned}
& \operatorname{Str}\left(\chi^{2}\right)=2 \operatorname{tr}\left(\Theta \Theta_{*}\right)=-2 \operatorname{tr}\left(\Sigma \Theta^{\dagger} \Theta\right), \\
& \operatorname{Str}\left(\Sigma_{+} \chi \widetilde{K}_{8} \chi^{\prime t} K_{8}\right)=-\operatorname{tr}\left(\Sigma \Theta K \Theta^{\prime t} K\right)-\operatorname{tr}\left(\Sigma \Theta^{\dagger} K \Theta^{\prime \dagger, t} K\right),
\end{aligned}
$$

to write the quadratic Hamiltonian in the form

$\mathcal{H}_{2}=\frac{1}{2} p_{M}^{2}+\frac{1}{2} x_{M}^{2}+\frac{\tilde{\lambda}}{2} x_{M}^{\prime 2}-\frac{\kappa}{2} \sqrt{\tilde{\lambda}}\left(\operatorname{tr}\left(\Sigma \Theta K \Theta^{\prime t} K\right)+\operatorname{tr}\left(\Sigma \Theta^{\dagger} K \Theta^{\prime \dagger, t} K\right)\right)-\operatorname{tr}\left(\Sigma \Theta^{\dagger} \Theta\right)$.

Then by using

$\operatorname{tr} \Sigma \Theta^{\dagger} \Theta=-\frac{1}{2} \operatorname{tr}\left(\eta^{\dagger} \eta+\theta^{\dagger} \theta\right)$

$\operatorname{tr} \Sigma \Theta K \Theta^{\prime t} K=\frac{1}{2} \operatorname{tr}\left(-\eta \eta^{\prime}+\theta^{\dagger} \theta^{\prime \dagger}\right), \quad \operatorname{tr} \Sigma \Theta^{\dagger} K \Theta^{\prime \dagger, t} K=\frac{1}{2} \operatorname{tr}\left(\eta^{\dagger} \eta^{\prime \dagger}-\theta \theta^{\prime}\right)$,

we obtain (5.20).

Quartic Hamiltonian. We first use the following relations,

$$
\begin{aligned}
& \operatorname{Str}\left(\chi^{\prime} \chi^{\prime}\right)=-2 \operatorname{tr}\left(\Sigma \Theta^{\prime} \Theta^{\prime \dagger}\right), \quad \operatorname{Str}\left(\chi \chi^{\prime} \chi \chi^{\prime}\right)=\operatorname{tr}\left(\Theta^{\prime \dagger} \Theta^{\prime \prime} \Theta^{\prime \dagger}-\Theta^{\dagger} \Theta^{\prime} \Theta^{\dagger} \Theta^{\prime}\right), \\
& \operatorname{Str}\left(\chi^{2} \chi^{\prime 2}\right)=\operatorname{tr}\left(\Theta \Theta^{\dagger} \Theta^{\prime} \Theta^{\prime \dagger}-\Theta^{\dagger} \Theta \Theta^{\prime \dagger} \Theta^{\prime}\right), \\
& \operatorname{Str}\left(\left(\chi \chi^{\prime}-\chi^{\prime} \chi\right) K_{8}\left(\chi \chi^{\prime}-\chi^{\prime} \chi\right)^{t} K_{8}\right)=\operatorname{tr}\left(\left(\Theta^{\prime \dagger}-\Theta^{\prime} \Theta^{\dagger}\right) K\left(\Theta \Theta^{\prime \dagger}-\Theta^{\prime} \Theta^{\dagger}\right)^{t} K\right. \\
& \left.-\left(\Theta^{\dagger} \Theta^{\prime}-\Theta^{\prime \dagger} \Theta\right) K\left(\Theta^{\dagger} \Theta^{\prime}-\Theta^{\prime \dagger} \Theta\right)^{t} K\right), \\
& \operatorname{Str}\left(\chi \widetilde{K}_{8} \chi^{\prime t} K_{8} \chi \widetilde{K}_{8} \chi^{\prime t} K_{8}\right)=\operatorname{tr}\left(K \Theta^{\prime \dagger, t} K \Theta^{\dagger} K \Theta^{\prime \dagger, t} K \Theta^{\dagger}\right)-\operatorname{tr}\left(K \Theta^{\prime t} K \Theta K \Theta^{\prime t} K \Theta\right), \\
& x_{M} x_{N} \operatorname{Str}\left(\Sigma_{M} \chi^{\prime} \Sigma_{N} \chi^{\prime}\right)=-2 \mathrm{i} Z_{m} Y_{n} \operatorname{tr}\left(\Sigma \Gamma_{m} \Theta^{\prime} \Gamma_{n} \Theta^{\prime \dagger}\right), \\
& x_{M}^{\prime} x_{N} \operatorname{Str}\left(\left[\Sigma_{M}, \Sigma_{N}\right]\left(\chi \chi^{\prime}-\chi^{\prime} \chi\right)\right)=-Z_{m}^{\prime} Z_{n} \operatorname{tr}\left(\Sigma\left[\Gamma_{m}, \Gamma_{n}\right]\left(\Theta \Theta^{\prime \dagger}-\Theta^{\prime} \Theta^{\dagger}\right)\right) \\
& +Y_{m}^{\prime} Y_{n} \operatorname{tr}\left(\Sigma\left[\Gamma_{m}, \Gamma_{n}\right]\left(\Theta^{\dagger} \Theta^{\prime}-\Theta^{\prime \dagger} \Theta\right)\right),
\end{aligned}
$$


$\left(x_{N} p_{M}\right)^{\prime} \operatorname{Str}\left(\left[\Sigma_{N}, \Sigma_{M}\right]\left(\widetilde{K}_{8} \chi^{t} K_{8} \chi-\chi \widetilde{K}_{8} \chi^{t} K_{8}\right)\right)$

$$
\begin{aligned}
= & 2\left(Z_{n} P_{m}^{z}\right)^{\prime} \operatorname{tr}\left(\left[\Gamma_{n}, \Gamma_{m}\right]\left(K \Theta^{\dagger, t} K \Theta^{\dagger}+\Theta K \Theta^{t} K\right)\right) \\
& -2\left(Y_{n} P_{m}^{y}\right)^{\prime} \operatorname{tr}\left(\left[\Gamma_{n}, \Gamma_{m}\right]\left(K \Theta^{t} K \Theta+\Theta^{\dagger} K \Theta^{\dagger, t} K\right)\right),
\end{aligned}
$$

to write the quartic Hamiltonians $\mathcal{H}_{b f}$ and $\mathcal{H}_{f f}$ in the form

$$
\begin{aligned}
\mathcal{H}_{b f}=\frac{1}{2 p_{+}}[- & 2 \tilde{\lambda}\left(z^{2}-y^{2}\right) \operatorname{tr}\left(\Sigma \Theta^{\prime} \Theta^{\dagger \dagger}\right)+4 \mathrm{i} \tilde{\lambda} Z_{m} Y_{n} \operatorname{tr}\left(\Sigma \Gamma_{m} \Theta^{\prime} \Gamma_{n} \Theta^{\prime \dagger}\right) \\
& -\frac{\tilde{\lambda}}{2} \operatorname{tr}\left(Z_{m}^{\prime} Z_{n} \Sigma\left[\Gamma_{m}, \Gamma_{n}\right]\left(\Theta \Theta^{\prime \dagger}-\Theta^{\prime} \Theta^{\dagger}\right)-Y_{m}^{\prime} Y_{n} \Sigma\left[\Gamma_{m}, \Gamma_{n}\right]\left(\Theta^{\dagger} \Theta^{\prime}-\Theta^{\prime \dagger} \Theta\right)\right) \\
& +\frac{\mathrm{i} \kappa}{2} \sqrt{\tilde{\lambda}} \operatorname{tr}\left(\left(Z_{n} P_{m}^{z}\right)^{\prime}\left[\Gamma_{n}, \Gamma_{m}\right]\left(K \Theta^{\dagger, t} K \Theta^{\dagger}+\Theta K \Theta^{t} K\right)\right. \\
& \left.\left.-\left(Y_{n} P_{m}^{y}\right)^{\prime}\left[\Gamma_{n}, \Gamma_{m}\right]\left(K \Theta^{t} K \Theta+\Theta^{\dagger} K \Theta^{\dagger, t} K\right)\right)\right]
\end{aligned}
$$

$\mathcal{H}_{f f}=\frac{1}{2 p_{+}}\left[-\frac{\tilde{\lambda}}{2} \operatorname{tr}\left(\Theta \Theta^{\prime \dagger} \Theta \Theta^{\prime \dagger}-\Theta^{\dagger} \Theta^{\prime} \Theta^{\dagger} \Theta^{\prime}+\Theta^{\prime} \Theta^{\dagger} \Theta^{\prime} \Theta^{\prime \dagger}-\Theta^{\dagger} \Theta \Theta^{\prime \dagger} \Theta^{\prime}\right)\right.$

$-\frac{\tilde{\lambda}}{4} \operatorname{tr}\left(\left(\Theta \Theta^{\prime \dagger}-\Theta^{\prime} \Theta^{\dagger}\right) K\left(\Theta \Theta^{\prime \dagger}-\Theta^{\prime} \Theta^{\dagger}\right)^{t} K\right.$

$\left.-\left(\Theta^{\dagger} \Theta^{\prime}-\Theta^{\prime \dagger} \Theta\right) K\left(\Theta^{\dagger} \Theta^{\prime}-\Theta^{\prime \dagger} \Theta\right)^{t} K\right)$

$\left.-\tilde{\lambda} \operatorname{tr}\left(K \Theta^{\prime \dagger, t} K \Theta^{\dagger} K \Theta^{\prime \dagger, t} K \Theta^{\dagger}-K \Theta^{\prime t} K \Theta K \Theta^{\prime t} K \Theta\right)\right]$.

Note that we used

$z_{m} \gamma_{m}=Z_{m} \Gamma_{m}, \quad y_{m} \gamma_{m}=Y_{m} \Gamma_{m}, \quad p_{m}^{y} \gamma_{m}=2 P_{m}^{y} \Gamma_{m}, \quad p_{m}^{y} \gamma_{m}=2 P_{m}^{y} \Gamma_{m}$.

Then by using

$$
\begin{aligned}
& \operatorname{tr} \Sigma\left(\Theta^{\prime \dagger} \Theta^{\prime}\right)=-\frac{1}{2} \operatorname{tr}\left(\eta^{\prime \dagger} \eta^{\prime}+\theta^{\prime \dagger} \theta^{\prime}\right), \\
& \operatorname{tr} \Sigma \Gamma_{m} \Gamma_{n}\left(\Theta \Theta^{\prime \dagger}-\Theta^{\prime} \Theta^{\dagger}\right)=\operatorname{tr}\left(\mathcal{P}_{+} \Gamma_{m} \Gamma_{n}\left(\eta \eta^{\prime \dagger}-\eta^{\prime} \eta^{\dagger}\right)-\mathcal{P}_{-} \Gamma_{m} \Gamma_{n}\left(\theta^{\dagger} \theta^{\prime}-\theta^{\prime \dagger} \theta\right)\right), \\
& \operatorname{tr} \Sigma \Gamma_{m} \Gamma_{n}\left(\Theta^{\dagger} \Theta^{\prime}-\Theta^{\prime \dagger} \Theta\right)=\operatorname{tr}\left(-\mathcal{P}_{-} \Gamma_{m} \Gamma_{n}\left(\eta^{\dagger} \eta^{\prime}-\eta^{\prime \dagger} \eta\right)+\mathcal{P}_{+} \Gamma_{m} \Gamma_{n}\left(\theta \theta^{\prime \dagger}-\theta^{\prime} \theta^{\dagger}\right)\right), \\
& \operatorname{tr} \Sigma \Gamma_{m} \Theta^{\prime} \Gamma_{n} \Theta^{\prime \dagger}=\operatorname{tr}\left(-\mathcal{P}_{-} \Gamma_{m} \eta^{\prime} \Gamma_{n} \theta^{\prime}+\mathcal{P}_{+} \Gamma_{m} \theta^{\prime \dagger} \Gamma_{n} \eta^{\prime \dagger}\right), \\
& \operatorname{tr}\left(\left[\Gamma_{n}, \Gamma_{m}\right]\left(K \Theta^{\dagger, t} K \Theta^{\dagger}+\Theta K \Theta^{t} K\right)\right) \\
& =-\operatorname{tr} \mathcal{P}_{+}\left[\Gamma_{n}, \Gamma_{m}\right]\left(\eta^{\dagger} \eta^{\dagger}+\eta \eta\right)-\operatorname{tr} \mathcal{P}_{-}\left[\Gamma_{n}, \Gamma_{m}\right]\left(\theta^{\dagger} \theta^{\dagger}+\theta \theta\right), \\
& \operatorname{tr}\left(\left[\Gamma_{n}, \Gamma_{m}\right]\left(K \Theta^{t} K \Theta+\Theta^{\dagger} K \Theta^{\dagger, t} K\right)\right) \\
& =-\operatorname{tr} \mathcal{P}_{-}\left[\Gamma_{n}, \Gamma_{m}\right]\left(\eta^{\dagger} \eta^{\dagger}+\eta \eta\right)-\operatorname{tr} \mathcal{P}_{+}\left[\Gamma_{n}, \Gamma_{m}\right]\left(\theta^{\dagger} \theta^{\dagger}+\theta \theta\right), \\
& \text { we obtain }(5.23) . \\
& \operatorname{Tofind} H_{f f} \operatorname{we} \operatorname{need} \\
& \operatorname{tr}\left(\Theta \Theta^{\prime \dagger} \Theta \Theta^{\prime \dagger}-\Theta^{\dagger} \Theta^{\prime} \Theta^{\dagger} \Theta^{\prime}+\Theta \Theta^{\dagger} \Theta^{\prime} \Theta^{\prime \dagger}-\Theta^{\dagger} \Theta \Theta^{\prime \dagger} \Theta^{\prime}\right) \\
& =\operatorname{tr}\left(\mathcal{P}_{+} \eta \eta^{\prime \dagger} \eta \eta^{\prime \dagger}-\mathcal{P}_{-} \eta^{\dagger} \eta^{\prime} \eta^{\dagger} \eta^{\prime}+\mathcal{P}_{+} \eta \eta^{\dagger} \eta^{\prime} \eta^{\prime \dagger}-\mathcal{P}_{-} \eta^{\dagger} \eta \eta^{\prime \dagger} \eta^{\prime}\right. \\
& \left.\quad-\mathcal{P}_{+} \theta \theta^{\prime \dagger} \theta \theta^{\prime \dagger}+\mathcal{P}_{-} \theta^{\dagger} \theta^{\prime} \theta^{\dagger} \theta^{\prime}-\mathcal{P}_{+} \theta \theta^{\dagger} \theta^{\prime} \theta^{\prime \dagger}+\mathcal{P}_{-} \theta^{\dagger} \theta \theta^{\prime \dagger} \theta^{\prime}\right), \\
& \operatorname{tr}\left(\left(\Theta \Theta^{\prime \dagger}-\Theta^{\prime} \Theta^{\dagger}\right) K\left(\Theta \Theta^{\prime \dagger}-\Theta^{\prime} \Theta^{\dagger}\right)^{t} K-\left(\Theta^{\dagger} \Theta^{\prime}-\Theta^{\prime \dagger} \Theta\right) K\left(\Theta^{\dagger} \Theta^{\prime}-\Theta^{\prime \dagger} \Theta\right)^{t} K\right) \\
& =\operatorname{tr} \Sigma\left(\left(\eta \eta^{\prime \dagger}-\eta^{\prime} \eta^{\dagger}\right)\left(\eta^{\prime \dagger} \eta-\eta^{\dagger} \eta^{\prime}\right)-\left(\theta^{\prime \dagger} \theta-\theta^{\dagger} \theta^{\prime}\right)\left(\theta \theta^{\prime \dagger}-\theta^{\prime} \theta^{\dagger}\right)\right), \\
& \operatorname{tr}\left(K \Theta^{\prime \dagger, t} K \Theta^{\dagger} K \Theta^{\prime \dagger, t} K \Theta^{\dagger}-K \Theta^{\prime t} K \Theta K \Theta^{\prime t} K \Theta\right) \\
& =\operatorname{tr}\left(\mathcal{P}_{+} \eta^{\prime \dagger} \eta^{\dagger} \eta^{\prime \dagger} \eta^{\dagger}-\mathcal{P}_{-} \eta^{\prime} \eta \eta^{\prime} \eta-\mathcal{P}_{+} \theta^{\prime \dagger} \theta^{\dagger} \theta^{\prime \dagger} \theta^{\dagger}+\mathcal{P}_{-} \theta^{\prime} \theta \theta^{\prime} \theta\right) .
\end{aligned}
$$


By using these formulae we find the following expression for $H_{f f}$,

$$
H_{f f}=H_{f f}(\eta)-H_{f f}(\theta)
$$

where

$$
\begin{aligned}
H_{f f}(\eta)=\frac{\tilde{\lambda}}{2 p_{+}} \operatorname{tr}\left[-\frac{1}{2}\left(\mathcal{P}_{+} \eta \eta^{\prime \dagger} \eta \eta^{\prime \dagger}-\mathcal{P}_{-} \eta^{\dagger} \eta^{\prime} \eta^{\dagger} \eta^{\prime}+\mathcal{P}_{+} \eta \eta^{\dagger} \eta^{\prime} \eta^{\prime \dagger}-\mathcal{P}_{-} \eta^{\dagger} \eta \eta^{\prime \dagger} \eta^{\prime}\right)\right. \\
\left.-\frac{1}{4} \Sigma\left(\eta \eta^{\prime \dagger}-\eta^{\prime} \eta^{\dagger}\right)\left(\eta^{\prime \dagger} \eta-\eta^{\dagger} \eta^{\prime}\right)-\left(\mathcal{P}_{+} \eta^{\prime \dagger} \eta^{\dagger} \eta^{\prime \dagger} \eta^{\dagger}-\mathcal{P}_{-} \eta^{\prime} \eta \eta^{\prime} \eta\right)\right]
\end{aligned}
$$

It can be cast in the form

$$
\begin{aligned}
H_{f f}(\eta)=\frac{\tilde{\lambda}}{2 p_{+}} \operatorname{tr} & {\left[-\frac{1}{2} \Sigma\left(\eta^{\prime \dagger} \eta \eta^{\prime \dagger} \eta+\eta^{\dagger} \eta^{\prime} \eta^{\dagger} \eta^{\prime}+\eta^{\prime \dagger} \eta^{\dagger} \eta^{\prime \dagger} \eta^{\dagger}+\eta^{\prime} \eta \eta^{\prime} \eta\right)\right.} \\
& \left.-\frac{1}{4}\left(\eta \eta^{\prime \dagger} \eta \eta^{\prime \dagger}-\eta^{\dagger} \eta^{\prime} \eta^{\dagger} \eta^{\prime}+\eta \eta^{\dagger} \eta^{\prime} \eta^{\prime \dagger}-\eta^{\dagger} \eta \eta^{\prime \dagger} \eta^{\prime}+2 \eta^{\prime \dagger} \eta^{\dagger} \eta^{\prime \dagger} \eta^{\dagger}-2 \eta^{\prime} \eta \eta^{\prime} \eta\right)\right]
\end{aligned}
$$

By using the formula

$$
\operatorname{tr} \Gamma_{a} \Gamma_{5-b} \Gamma_{c} \Gamma_{5-d}=\delta_{a b} \delta_{c d}+\delta_{a d} \delta_{b c}-\delta_{a c} \delta_{b d},
$$

one can check that the second line in (D.6) vanishes, and, therefore, $H_{f f}(\eta)$ takes the form (5.25).

\section{References}

[1] Metsaev R R and Tseytlin A A 1998 Type IIB superstring action in AdS(5) x s(5) background Nucl. Phys. B 533109 (preprint hep-th/9805028)

[2] Schwarz J H 1983 Covariant field equations of chiral N =2 D = 10 supergravity Nucl. Phys. B 226269

[3] Blau M, Figueroa-O'Farrill J, Hull C and Papadopoulos G 2002 A new maximally supersymmetric background of IIB superstring theory J. High Energy Phys. JHEP01(2002)047 (preprint hep-th/0110242)

[4] Blau M, Figueroa-O'Farrill J, Hull C and Papadopoulos G 2002 Penrose limits and maximal supersymmetry Class. Quantum Grav. 19 L87 (preprint hep-th/0201081)

[5] Metsaev R R 2002 Type IIB Green-Schwarz superstring in plane wave Ramond-Ramond background Nucl. Phys. B 62570 (preprint hep-th/0112044)

[6] Maldacena J M 1998 The large N limit of superconformal field theories and supergravity Adv. Theor. Math. Phys. 2231

Maldacena J M 1999 Int. J. Theor. Phys. 381113 (preprint hep-th/9711200)

[7] Gubser S S, Klebanov I R and Polyakov A M 1998 Gauge theory correlators from non-critical string theory Phys. Lett. B 428105 (preprint hep-th/9802109)

[8] Witten E 1998 Anti-de Sitter space and holography Adv. Theor. Math. Phys. 2253 (preprint hep-th/9802150)

[9] Beisert N 2005 The dilatation operator of N = 4 super Yang-Mills theory and integrability Phys. Rep. 4051 (preprint hep-th/0407277)

Zarembo K 2004 Semiclassical Bethe ansatz and AdS/CFT C. R. Phys. 51081

Zarembo K 2005 Fortschr. Phys. 53647 (preprint hep-th/0411191)

Plefka J 2005 Spinning strings and integrable spin chains in the AdS/CFT correspondence Preprint hep-th/0507136

[10] Peeters K, Plefka J and Zamaklar M 2004 Splitting spinning strings in AdS/CFT J. High Energy Phys. JHEP11(2004)054 (preprint hep-th/0410275)

Peeters K, Plefka J and Zamaklar M 2005 Splitting strings and chains Fortschr. Phys. 53640 (preprint hep-th/0501165)

[11] Beisert N, Kristjansen C and Staudacher M 2003 The dilatation operator of N = 4 super Yang-Mills theory Nucl. Phys. B 664131 (preprint hep-th/0303060)

[12] Minahan J A and Zarembo K 2003 The Bethe-ansatz for N = 4 super Yang-Mills J. High Energy Phys. JHEP03(2003)013 (preprint hep-th/0212208) 
[13] Beisert N 2004 The su(2/3) dynamic spin chain Nucl. Phys. B 682487 (preprint hep-th/0310252)

[14] Eden B, Jarczak C and Sokatchev E 2005 Three-loop test of the dilatation operator and integrability in $=4$ SYM Fortschr. Phys. $\mathbf{5 3} 610$

[15] Beisert N, Dippel V and Staudacher M 2004 A novel long range spin chain and planar N = 4 super Yang-Mills J. High Energy Phys. JHEP07(2004)075 (preprint hep-th/0405001)

[16] Beisert N and Staudacher M 2005 Long-range PSU(2,214) Bethe ansätze for gauge theory and strings Nucl. Phys. B 7271 (preprint hep-th/0504190)

[17] Rej A, Serban D and Staudacher M 2005 Planar N = 4 gauge theory and the Hubbard model Preprint hep-th/0512077

[18] Berenstein D, Maldacena J M and Nastase H 2002 Strings in flat space and pp waves from $\mathrm{N}=4$ super Yang-Mills J. High Energy Phys. JHEP04(2002)013 (preprint hep-th/0202021)

[19] Callan C G, Lee H K, McLoughlin T, Schwarz J H, Swanson I and Wu X 2003 Quantizing string theory in $\mathrm{AdS}_{5} \times \mathrm{S}^{5}$ : beyond the pp-wave $N$ ucl. Phys. B 6733 (preprint hep-th/0307032)

Callan C G, McLoughlin T and Swanson I 2004 Holography beyond the Penrose limit Nucl. Phys. B 694115 (preprint hep-th/0404007)

C G Callan, McLoughlin T and Swanson I 2004 Higher impurity AdS/CFT correspondence in the near-BMN limit Nucl. Phys. B 700271 (preprint hep-th/0405153)

[20] Gubser S S, Klebanov I R and Polyakov A M 2002 A semi-classical limit of the gauge/string correspondence Nucl. Phys. B 63699 (preprint hep-th/0204051)

[21] Frolov $\mathrm{S}$ and Tseytlin A A 2002 Semiclassical quantization of rotating superstring in $\mathrm{AdS}_{5} \times \mathrm{S}^{5}$ J. High Energy Phys. JHEP06(2002)007 (preprint hep-th/0204226)

Frolov S and Tseytlin A A 2003 Multi-spin string solutions in $\mathrm{AdS}_{5} \times \mathrm{S}^{5}$ Nucl. Phys. B 66877 (preprint hep-th/0304255)

[22] Frolov S and Tseytlin A A 2003 Quantizing three-spin string solution in $\mathrm{AdS}_{5} \times \mathrm{S}^{5}$ J. High Energy Phys. JHEP07(2003)016 (preprint hep-th/0306130)

Arutyunov G, Russo J and Tseytlin A A 2004 Spinning strings in $\mathrm{AdS}_{5} \times \mathrm{S}^{5}$ : new integrable system relations Phys. Rev. D 69086009 (preprint hep-th/0311004)

Frolov S A, Park I Y and Tseytlin A A 2005 On one-loop correction to energy of spinning strings in $\mathrm{S}^{5}$ Phys. Rev. D 71026006 (preprint hep-th/0408187)

Park I Y, Tirziu A and Tseytlin A A 2005 Spinning strings in $\mathrm{AdS}_{5} \times \mathrm{S}^{5}$ : one-loop correction to energy in SL(2) sector J. High Energy Phys. JHEP03(2005)013 (preprint hep-th/0501203)

[23] Bena I, Polchinski J and Roiban R 2004 Hidden symmetries of the $\mathrm{AdS}_{5} \times \mathrm{S}^{5}$ superstring Phys. Rev. D 69046002 (preprint hep-th/0305116)

[24] Arutyunov G, Frolov S and Staudacher M 2004 Bethe ansatz for quantum strings J. High Energy Phys. JHEP10(2004)016 (preprint hep-th/0406256)

[25] Schäfer-Nameki S, Zamaklar M and Zarembo K 2005 Quantum corrections to spinning strings in $\mathrm{AdS}_{5} \times \mathrm{S}^{5}$ and Bethe ansatz: a comparative study J. High Energy Phys. JHEP09(2005)051 (preprint hep-th/0507189)

Schäfer-Nameki S and Zamaklar M 2005 Stringy sums and corrections to the quantum string Bethe ansatz J. High Energy Phys. JHEP10(2005)044 (preprint hep-th/0509096)

Schäfer-Nameki S 2006 Exact expressions for quantum corrections to spinning strings Preprint hep-th/0602214

[26] Beisert N and Tseytlin A A 2005 On quantum corrections to spinning strings and Bethe equations Phys. Lett. B 629102 (preprint hep-th/0509084)

[27] Mann N and Polchinski J 2005 Bethe ansatz for a quantum supercoset sigma model Phys. Rev. D 72086002 (preprint hep-th/0508232)

Mann N and Polchinski J 2004 Finite density states in integrable conformal field theories Preprint hep-th/0408162

[28] Arutyunov G and Frolov S 2005 Uniform light-cone gauge for strings in $\mathrm{AdS}_{5} \times \mathrm{S}^{5}$ : solving su(1l1) sector preprint hep-th/0510208

[29] Goddard P, Goldstone J, Rebbi C and Thorn C B 1973 Quantum dynamics of a massless relativistic string Nucl. Phys. B 56109

[30] Beisert N 2005 Spin chain for quantum strings Fortschr. Phys. 53852 (preprint hep-th/0409054)

[31] Alday L F, Arutyunov G and Tseytlin A A 2005 On integrability of classical superstrings in $\mathrm{AdS}_{5} \times \mathrm{S}^{5} \mathrm{~J}$. High Energy Phys. JHEP07(2005)002 (preprint hep-th/0502240)

[32] Alday L F, Arutyunov G and Frolov S 2005 New integrable system of 2dim fermions from strings on $\mathrm{AdS}_{5} \times \mathrm{S}^{5}$ preprint hep-th/0508140

[33] Roiban R and Siegel W 2000 Superstrings on $\mathrm{AdS}_{5} \times \mathrm{S}^{5}$ supertwistor space J. High Energy Phys. JHEP11(2000)024 (preprint hep-th/0010104)

[34] Alday L F, Arutyunov G and Frolov S 2005 Green-Schwarz strings in TsT-transformed backgrounds Preprint hep-th/0512253 
[35] Lunin O and Maldacena J 2005 Deforming field theories with $U(1) \times U(1)$ global symmetry and their gravity duals J. High Energy Phys. JHEP05(2005)033 (preprint hep-th/0502086)

[36] Frolov S 2005 Lax pair for strings in Lunin-Maldacena background J. High Energy Phys. JHEP05(2005)069 (preprint hep-th/0503201)

[37] Frolov S A, Roiban R and Tseytlin A A 2005 Gauge-string duality for superconformal deformations of $N=4$ super Yang-Mills theory J. High Energy Phys. JHEP07(2005)045 (preprint hep-th/0503192)

de Mello Koch R, Murugan J, Smolic J and Smolic M 2005 Deformed PP-waves from the Lunin-Maldacena background J. High Energy Phys. JHEP08(2005)072 (preprint hep-th/0505227)

Mateos T 2005 Marginal deformation of N = 4 SYM and Penrose limits with continuum spectrum J. High Energy Phys. JHEP08(2005)026 (preprint hep-th/0505243)

Frolov S A, Roiban R and Tseytlin A A 2005 Gauge-string duality for (non)supersymmetric deformations of $\mathrm{N}=4$ super Yang-Mills theory Nucl. Phys. B 7311 (preprint hep-th/0507021)

[38] Metsaev R R, Thorn C B and Tseytlin A A 2001 Light-cone superstring in AdS space-time Nucl.Phys. B 596151 (preprint hep-th/0009171)

[39] Kruczenski M, Ryzhov A V and Tseytlin A A 2004 Large spin limit of $\mathrm{AdS}_{5} \times \mathrm{S}^{5}$ string theory and low energy expansion of ferromagnetic spin chains Nucl. Phys. B 6923 (preprint hep-th/0403120)

Kruczenski M and Tseytlin A A 2004 Semiclassical relativistic strings in $S^{5}$ and long coherent operators in N = 4 SYM theory J. High Energy Phys. JHEP09(2004)038 (preprint hep-th/0406189)

[40] Minahan J A, Tirziu A and Tseytlin A A $20061 / J$ corrections to semiclassical AdS/CFT states from quantum Landau-Lifshitz model Nucl. Phys. B 735127 (preprint hep-th/0509071)

Minahan J A, Tirziu A and Tseytlin A A $20051 / J^{2}$ corrections to BMN energies from the quantum long range Landau-Lifshitz model J. High Energy Phys. JHEP11(2005)031 (preprint hep-th/0510080)

Tirziu A 2006 Quantum Landau-Lifshitz model at four loops: $1 / J$ and $1 / J^{2}$ corrections to BMN energies Preprint hep-th/0601139

[41] Arutyunov G and Frolov S 2005 Integrable Hamiltonian for classical strings on $\mathrm{AdS}_{5} \times \mathrm{S}^{5}$ J. High Energy Phys. JHEP02(2005)059 (preprint hep-th/0411089)

[42] McLoughlin T and Swanson I 2004 N-impurity superstring spectra near the pp-wave limit Nucl.Phys. B 70286 (preprint hep-th/0407240)

[43] Staudacher M 2005 The factorized S-matrix of CFT/AdS J. High Energy Phys. JHEP05(2005)054 (preprint hep-th/0412188)

[44] Kazakov V A, Marshakov A, Minahan J A and Zarembo K 2004 Classical/quantum integrability in AdS/CFT J. High Energy Phys. JHEP05(2004)024 (preprint hep-th/0402207)

[45] Kazakov V A and Zarembo K 2004 Classical/quantum integrability in non-compact sector of AdS/CFT J. High Energy Phys. JHEP10(2004)060 (preprint hep-th/0410105)

Beisert N, Kazakov V A and Sakai K 2004 Algebraic curve for the SO(6) sector of AdS/CFT Preprint hep-th/0410253

Beisert N, Kazakov V A, Sakai K and Zarembo K 2005 The algebraic curve of classical superstrings on $\mathrm{AdS}_{5} \times \mathrm{S}^{5}$ Preprint hep-th/0502226

Schäfer-Nameki S 2005 The algebraic curve of 1-loop planar $\mathrm{N}=4$ SYM Nucl. Phys. B 7143 (preprint hep-th/0412254)

[46] Minahan J A 2005 The SU(2) sector in AdS/CFT Fortschr. Phys. 53828 (preprint hep-th/0503143)

[47] Kruczenski M 2004 Spin chains and string theory Phys. Rev. Lett. 93161602 (preprint hep-th/0311203)

[48] Beisert N, Minahan J A, Staudacher M and Zarembo K 2003 Stringing spins and spinning strings J. High Energy Phys. JHEP09(2003)010 (preprint hep-th/0306139) 